\title{
LONG-DURATION, LOW-LUMINOSITY GAMMA-RAY BURSTS: TOWARDS A COMPREHENSIVE MODEL OF THE WEAKEST ENGINE-DRIVEN EXPLOSIONS
}

\author{
Christopher Michael Irwin \\ Irwin, Pennsylvania
}

B.S. Physics \& Astronomy, University of Pittsburgh, 2009

M.S. Astronomy, University of Virginia, 2012

A Dissertation Presented to the Graduate

Faculty of the University of Virginia

in Candidacy for the Degree of

Doctor of Philosophy

Department of Astronomy

University of Virginia

August 2016

Committee Members:

Professor Roger A. Chevalier

Professor Zhi-Yun Li

Professor Craig L. Sarazin

Professor Hank B. Thacker 
(C) Copyright by

Christopher Michael Irwin

All rights reserved

August 10, 2016 


\begin{abstract}
Long-duration, low-luminosity gamma-ray bursts (LLGRBs) are a peculiar subclass of gamma-ray bursts (GRBs) that are up to 5 orders of magnitude less luminous than typical bursts. The observation of Type Ic supernovae (SNe) accompanying LLGRBs supports the notion that, like typical GRBs, they are powered by the gravitational collapse of a massive star's core, but beyond this little is known about their origin, and the differences between the progenitors of LLGRBs and GRBs are unclear. In this dissertation, I present analytical and numerical modeling that aims to improve our understanding of the physical conditions leading to LLGRBs.

We consider first the unusual GRB 060218, a well-studied event that is prototypical of the long-duration LLGRB class. We present a model for this burst that can account for the prompt X-ray emission lasting for $\sim 3000 \mathrm{~s}$, the early optical peak lasting for hours, and the X-ray and radio afterglow lasting for several days. The basic ingredients of the model are a long-lived jet $\left(t_{j} \sim 3000 \mathrm{~s}\right)$ with an unusually low luminosity $\left(L_{j} \sim 10^{47} \mathrm{erg} \mathrm{s}^{-1}\right)$, a progenitor star surrounded by an extended $\left(\sim 10^{13} \mathrm{~cm}\right)$, optically thick, low-mass $\left(\sim 10^{-2} M_{\odot}\right)$ envelope, and a modest amount of dust $\left(A_{V} \sim 0.1\right)$ in the interstellar environment.

We fit the observed thermal component of the prompt X-ray emission with a simple photospheric model, and then calculate the nonthermal emission due to inverse Compton (IC) scattering of thermal photons from the external shocks of the jet. We find that this model can fit the observed nonthermal emission, if the conditions are such that the reverse shock dominates the emission. We show that the low-power jet can successfully traverse the progenitor star and extended envelope, and that it can also produce the observed radio emission via external shock synchrotron emission at late times. In the meantime, fast ejecta from the associated SN 2006aj shock the
\end{abstract}


extended envelope, which emits a burst of optical emission as it expands and cools, consistent with the early optical peak. We interpret the anomalous X-ray afterglow in GRB 060218 as a light echo from dust situated $\sim 30$ pc from the burst. Our results suggest that LLGRB progenitors are shrouded in dense circumstellar envelopes, and that they require both a jet and a supernova. Support for this view comes from Nakar (2015), who considered a different model for LLGRBs involving choked jets, but arrived at the same main conclusions.

Motivated by these results, we also perform numerical simulations of jets with standard GRB properties in stars with extended envelopes. We present analytical calculations that predict whether or not the jet escapes successfully from the envelope, and apply them to interpret the numerical results. We find that the jet-driven outflow is more well-confined by the envelope than previous analytical results suggested, leading to a flow that is far from spherically symmetric when it breaks out of the envelope. This is generally true for a wide range of physical parameters. Even though the outflow fails to sphericize in the envelope, the jet still pumps most of its energy into a hot, mildly relativistic cocoon that expands after breakout to produce a roughly spherical explosion outside the envelope. The dynamics are thus very different from the standard case of a bare WR star progenitor, where the jet does become spherical if choked, and the outflow remains beamed for a long time post-breakout. Our results have far-reaching implications for LLGRBs, suggesting a different event rate and different behavior of the breakout radiation compared to past analytical work that assumed spherical symmetry is achieved in the envelope. 


\section{Acknowledgments}

First and foremost, I would like to thank my advisor, Roger Chevalier. Without his training and guidance - and his endorsement of my sometimes far-fetched ideasthe completion of this research would not have been possible. His cautious way of thinking and enthusiasm for research have been an inspiration, and his knowledge never failed to impress. I greatly appreciate his willingness to let me operate largely independently, and his tremendous patience when I encountered any setbacks. All in all, he has been an outstanding advisor from whom I have learned a great deal. I am humbled to have learned supernova science from one of the best.

I also want to thank the other members of my thesis committee-Zhi-Yun Li, Craig Sarazin, and Hank Thacker - for being part of my defense, and for the helpful discussions and advice. A big thank you also goes out to Roger, Li, and Craig for the countless reference letters they submitted during the peak of job-hunting season. My undergraduate advisor, John Hillier, also played a major role in getting me into astronomy.

The entire UVA astronomy community has been wonderful, and there are too many names to mention, so a few will have to suffice. I want to thank Xiaping Tang, who has been a good friend and a constant source of advice, as well as the rest of Office 109 (past and present), and my fellow 6th years. Bo Zhao deserves thanks for introducing me to RENZO and helping to set up my first simulations. Ed Murphy was incredibly helpful in providing teaching advice when I taught a summer class a few years ago. Mark Whittle and Kelsey Johnson made my life much easier when I was organizing visits for prospective students.

I also thank the Jefferson Scholars Foundation for their extremely generous sup-

port of my graduate career (including funding the computer on which this entire 
thesis was typed), and for enabling me to travel to several conferences, including a three-week supernova and gamma-ray burst conference in Kyoto during fall of 2013. It was a pleasure to be a part of such a vibrant community. The opportunity to spend time such a diverse group of scientists and scholars was as enriching as it was fun. Shout outs to Rachael and Bill, who helped to coach me on my selection presentation, and to the other Fellows-Trey, Patrick, Allison, and Paul-who got to be coached by me instead.

Special thanks go to the Israeli astrophysics community-especially Udi Nakar, Re'em Sari, Tsvi Piran - for their hospitality during my visit in February 2016, the good science discussion, and the career advice. Rodolfo Barniol-Duran has also been a source of helpful discussion and pespective on life after grad school.

My parents have been amazingly supportive of my career path, and have always encouraged me in whatever I have pursued. I am deeply grateful for everything they have done for me.

I should also acknowledge to Charlottesville volleyball community. Volleyball has been a great source of fun and stress relief during my grad school career, and was the way I met many of my closest friends in town. Last but not least, shout outs to all of my friends and family back in Pittsburgh. The moral support you all provide has been invaluable. Yinz guys are the best.

I would like to dedicate this thesis to Nancy Irwin, David Hanley, and Tom Hanley, who are no longer with us, but would have been proud. 


\section{Table of contents}

$\begin{array}{ll}\text { Abstract } & \text { ii }\end{array}$

1 Introduction: GRB Essentials 1

1.1 Collapsars and the GRB-SN connection . . . . . . . . . . . 4

1.2 Dynamics of relativistic jets . . . . . . . . . . . . . 5

1.3 Radiation from relativistic jets I: The prompt phase . . . . . . . . 11

1.4 Radiation from relativistic jets II: The afterglow phase . . . . . . . 14

2 Jet or Shock Breakout? The Low-Luminosity GRB $060218 \quad 21$

$\begin{array}{ll}\text { Abstract } & 22\end{array}$

2.1 Introduction . . . . . . . . . . . . . . . . . . . 22

2.2 Overview of observations . . . . . . . . . . . . . . . . 27

2.3 Shock breakout or central engine? . . . . . . . . . . . . . . 32

2.4 A comprehensive model for GRB $060218 \ldots \ldots \ldots \ldots$

2.4.1 Prompt thermal emission . . . . . . . . . . . . . . . . . 35

2.4 .2 Extinction and absorption . . . . . . . . . . . . 40

$2.4 .3 \quad \mathrm{UV} /$ optical emission . . . . . . . . . . . . . . . . . . . . 42

2.4 .4 X-ray afterglow . . . . . . . . . . . . . . . . . . . . 45

2.4 .5 Radio afterglow . . . . . . . . . . . . . . . . . . 53

2.4 .6 Jet propagation . . . . . . . . . . . . . . . 62

2.4.7 Prompt nonthermal emission . . . . . . . . . . . . . . 71

2.5 Discussion . . . . . . . . . . . . . . . . . . . . . . . . . . . . 88

2.6 Conclusions . . . . . . . . . . . . . . . . . . . . . . . . . 96

3 Numerical Simulations of Relativistic Jets in Extended Circumstel$\begin{array}{ll}\text { lar Envelopes } & 101\end{array}$

$\begin{array}{ll}\text { Abstract } & 102\end{array}$

3.1 Introduction . . . . . . . . . . . . . . . . 102

3.2 Methods . . . . . . . . . . . . . . . . . . . . 105

3.2 .1 Grid setup . . . . . . . . . . . . . . . . 106

3.2 .2 Jet injection . . . . . . . . . . . . . . . . 107 
3.2.3 Star and envelope models . . . . . . . . . . . . . . . . 111

3.3 Analytical expectations . . . . . . . . . . . . . . . . . . . 112

3.4 Results . . . . . . . . . . . . . . . . . . . . . 118

3.4.1 Model t20M4R9a2: "Standard" parameters . . . . . . . . . . . 118

3.4.2 Model t20M4R90a0: The effect of the envelope density profile 124

3.4.3 Model t20M400R9a2: A high-mass envelope . . . . . . . . . . 124

3.4.4 Model t10M12R18a2: A short-lived jet in a large envelope . . 127

3.5 Discussion . . . . . . . . . . . . . . . . . . . . . . 129

3.6 Summary and Conclusions . . . . . . . . . . . . . . . . . 132

4 Future Work: The Ultra-Long Gamma-Ray Burst Connection 135

$\begin{array}{lr}\text { Abstract } & 136\end{array}$

4.1 Introduction . . . . . . . . . . . . . . . . 136

4.2 Background . . . . . . . . . . . . . . . . . . . . 137

4.3 Methodology . . . . . . . . . . . . . . . . . . . 142

4.4 Expected results and applications . . . . . . . . . . . . 145

5 Summary and Conclusions $\quad 149$

$\begin{array}{ll}\text { Appendices } & 155\end{array}$

A Shock Dynamics of a Relativistic Outflow Interacting with a PowerLaw CSM 156

B Inverse Compton Radiation from a Relativistic Outflow Interacting with a Power-Law CSM 161 


\section{Chapter 1}

\section{Introduction: GRB Essentials}

Since their discovery in the late 1960's (Klebesadel et al. 1973), gamma-ray bursts (GRBs) have been the subject of active astronomical research. Our understanding of GRBs has come a long way in the past 50 years: we now know that long GRBs (LGRBs) occur at cosmological distances; that they result from the collapse of the core of massive stars, and occur alongside supernovae (SNe); and that they involve beamed, relativistic outflows. However, some aspects of GRBs, in particular the radiation mechanism that powers their early emission, remain mysterious to this day.

Even as our understanding of standard GRBs remains incomplete, our observational capabilities are constantly improving, and the diversity of known $\gamma$-ray transients continues to increase. The NASA satellite Swift-for now, the primary driver of GRB discovery - has in recent years detected a number of new and unusual events. Some of these never-before-seen high-energy transients have satisfactory explanations, like those caused by the tidal shredding of stars by supermassive black holes (Krolik \& Piran 2011; Cenko et al. 2012). Others, such as GRBs with extremely low luminosity and/or long timescale (Campana et al. 2006; Liang et al. 2007; Levan et al. 2014)

are more perplexing. The types of GRBs known so far-short GRBs that last less 
than $1 \mathrm{~s}$, and long GRBs (LGRBs) that last $\sim 10-100 \mathrm{~s}$ - are thought to come from different progenitor systems (Nakar 2007; Woosley \& Bloom 2006), so it is natural to wonder whether these newly discovered GRB subtypes have a different origin as well. As Swift and future missions will undoubtedly turn up ever more of these peculiar events, a comprehensive theoretical framework to understand them is needed sooner rather than later.

Of particular interest among new GRB subtypes are low luminosity GRBs (LLGRBs) with especially long duration (e.g, Campana et al. 2006). These faint objects can only be observed nearby, but their proximity allows for much better observations of the accompanying $\mathrm{SN}$, and better constraints on the burst properties. Furthermore, the estimated rate per cosmic volume of LLGRBs is considerably higher than that of standard LGRBs (Soderberg et al. 2006), implying a more common type of progenitor as well. Gaining a better grasp on LLGRB progenitors is therefore critical to understanding the ways that massive stars end their lives. In addition, because long LLGRBs have much in common with typical long GRBs, anything we can learn about their radiative processes can provide crucial insight into the longstanding problem of the GRB radiation mechanism. Despite the many good reasons to care about LLGRBs, still little is known about their true nature, in part due to the small number of events observed so far.

This doctoral thesis aims to move us closer to an understanding of the origin of long LLGRBs that can inform future theoretical work and observations. We accomplish this through detailed analysis of the best-observed member of the long LLGRB class, GRB 060218. We attempt to construct a comprehensive analytical model of this burst that simultaneously explains its many peculiar features. Among the more robust conclusions of our model is the result that the progenitor of GRB 060218 must 
be shrouded in a low-mass envelope extending out to radii much larger than the central star. Following this lead, we also pursue numerical simulations that track the dynamics of a GRB jet as it plows into this extended envelope. The net result of these efforts is an emerging picture for LLGRBs wherein interaction of the jet and supernova with an unusual, extended progenitor structure produce the early optical and X-ray $/ \gamma$-ray signals, and also lead to rapid spherical expansion of the relativistic ejecta after breaking out of the envelope that produces radio emission consistent with observations at late times.

The structure of this thesis is as follows. In Chapter 1, we overview essential GRB theory and observations, covering four broad topic: the connection between GRBs and SNe (Section 1.1), the dynamics of GRB jets (Section 1.2), and the radiation from GRB jets at early (Section 1.3) and late (Section 1.4) times. We mostly focus on standard GRBs to set the stage for later chapters, but we highlight important differences between typical GRBs and LLGRBs where appropriate, and delve into detail on highly relevant topics. In Chapter 2, we discuss possible origins of the peculiar long-duration, low-luminosity GRB 060218. We give background on the the topic of LLGRBs, summarize the work that has been done so far, and point out some problems with existing LLGRB models. Then we overview observations of GRB 060218, and consider a comprehensive analytical model that can account for many of its unusual features. In Chapter 3, we discuss the topic of jet propagation in an extended circumstellar envelope, which is applicable to long-duration GRBs. We present the results of numerical simulations that study the dynamical effects induced by this envelope, and discuss implications for models of LLGRBs, emphasizing differences from the standard case of a bare star. In Chapter 4, we discuss a possible future research project investigating ultra-long GRBs, another unusual type of gamma-ray transient 
that has some characteristics in common with LLGRBs. Finally, we summarize our conclusions in Chapter 5 .

\subsection{Collapsars and the GRB-SN connection}

The standard model for long GRBs is the "collapsar model" (MacFadyen \& Woosley 1999), in which the core of a rapidly rotating massive star collapses to form a compact

object (the "central engine") that launches a relativistic jet as it accretes infalling stellar material. This jet then tunnels through the progenitor star and goes on to produce the high-energy GRB emission after it emerges from the stellar surface. The compact object could be a black hole, or a highly magnetized neutron star - a "magnetar." The gravitational energy released from the collapse of the stellar core also powers a supernova explosion.

Several theoretical clues point to the collapsar scenario. First, the light curves of typical long GRBs vary on a timescale of $\delta t \sim 1 \mathrm{~ms}$. This suggests that the size of the emitting region is $\lesssim c \delta t$, implying a compact object (Piran 2004). On the other hand, the duration of long bursts, 10-100 s, is much longer than $\delta t$, implying that at least two time scales operate in the engine (Nakar \& Piran 2002). A reasonable way to obtain a second, longer timescale is to place an accretion disk around the compact object. Accretion onto compact objects is also known to produce relativistic jets (e.g. Blandford \& Znajek 1977), and GRB observations indeed show evidence of relativistic ejecta. Because, the GRB ejecta have a large kinetic energy $\left(\sim 10^{51} \mathrm{ergs}\right)$, a massive $\left(\sim 10^{-1} M_{\odot}\right)$ accretion disk is needed (Piran 2004). The above considerations point to accretion of a massive disk onto a compact object, but this can occur in various astrophysical systems, including binary systems with two compact objects, blackhole/He-star binaries, and the collapse of massive stars. Of these options, however, 
only stellar collapse can produce the timescale and accreted mass required for long GRBs (Narayan et al. 2001).

While the theoretical arguments are strong, the true smoking-gun evidence for the collapsar model comes from the connection between GRBs and SNe, which was cemented by the 1998 discovery of a GRB and SN coincident in time and space (Galama et al. 1998). Since then, many long GRBs have been associated with core-collapse SNe of Type Ic (Woosley \& Bloom 2006), solidifying the link between long GRBs and the collapse of massive stars. The fact that GRBs are correlated to star-forming galaxies (Christensen et al. 2004) further strengthens the connection. The available data are consistent with the idea that SNe accompany all long GRBs, although this cannot be conclusively proven (Woosley \& Bloom 2006). However, the reverse is not true: in fact, most SN progenitors do not produce GRBs. What determines whether or not a GRB accompanies the death of a massive star is a crucial open question.

SNe that are asssociated with GRBs often differ from from ordinary Type Ic SNe. Specifically, they belong the subclass of broad lined Type Ic SNe, so named because they have broad spectral lines indicating high outflow velocities. In addition, many GRB-SNe have a high explosion energy of up to $10^{52}$ ergs (Woosley \& Bloom 2006). While the SNe associated with LLGRBs are also broad-lined, their energies tend to be closer to the standard SN value of $10^{51} \mathrm{ergs}$ (e.g., Campana et al. 2006). Nonetheless, the SN may play an important role in early emission from LLGRBs, as we shall see in Section 2.4.3.

\subsection{Dynamics of relativistic jets}

Relativistic jets are common in many astrophysical environments, such as active galactic nuclei and GRBs, and accordingly have been the subject of much active research. 
The basic physical picture involves a jet of luminosity $L_{j}$ and opening angle $\theta_{0}$ injected into an external medium of density $\rho_{a}$. As the jet propagates, a double bow shock structure forms at the interface of the jet and the ambient medium. This forwardreverse shock system is called the "head" of the jet. Within the head, jet material entering through the reverse shock and ambient matter entering through the forward shock are separated by a contact discontinuity. Due to a steep pressure gradient, the shocked gas flows sideways out of the head, creating a high-pressure region of spent material called the "cocoon." The cocoon can be divided into an outer cocoon composed of shocked ambient gas, and an inner cocoon made of shocked jet gas.

If the cocoon pressure is sufficiently high, it drives an oblique shock into the expanding jet outflow. This oblique shock deflects the jet outflow, which is initially streaming in the radial direction, back towards the jet axis, while also increasing pressure in the jet. This process, known as collimation, substantially reduces the opening angle of the jet. We refer to the material above and below the collimation shock as the shocked jet and the unshocked jet, respectively. A steady state is eventually reached when the shocked jet pressure $p_{j}$ and the cocoon pressure $p_{c}$ become equal.

The speed of the head can be derived by balancing the ram pressure exerted by the jet on the reverse shock with the ram pressure exerted by the ambient medium on the forward shock (Matzner 2003), with the result

$$
p_{j}+h_{j} \rho_{j} \Gamma_{j}^{2} \Gamma_{h}^{2}\left(\beta_{j}-\beta_{h}\right)^{2} c^{2}=p_{a}+h_{a} \rho_{a} \Gamma_{h}^{2} \beta_{h}^{2} c^{2},
$$

where $\rho$ is density, $p$ is pressure, $\beta c$ is velocity, $\Gamma \equiv\left(1-\beta^{2}\right)^{-1 / 2}$ is Lorentz factor, $h=1+4 p / \rho c^{2}$ is enthalpy per unit rest mass energy in a relativistic gas, and $c$ is the speed of light. The subscripts $a, j$, and $h$ refer to the ambient medium, 
jet, and head respectively. The typical case for GRBs is that the reverse shock is strong, and the ambient medium is non-relativistic, leading to the approximations $p_{j} \ll \rho_{j} h_{j} \Gamma_{j}^{2} \Gamma_{h}^{2}\left(\beta_{j}-\beta_{h}\right)^{2}, p_{a} \ll \rho_{a} c^{2}$, and $h_{a} \approx 1$. Additionally, the jet is typically highly relativistic, so that $\beta_{j}$ can be set to 1 . In this case, equation 1.1 can be solved in the limit of a Newtonian head $\left(\beta_{h} \ll 1\right)$ or an ultra-relativistic head $\left(\Gamma_{h} \gg 1\right)$, with the result (Matzner 2003)

$$
\beta_{h}=\tilde{L}^{1 / 2}
$$

or

$$
\Gamma_{h}^{2}=\frac{1}{2} \tilde{L}^{1 / 2}
$$

where $\tilde{L}$ is a dimensionless parameter defined by

$$
\tilde{L} \equiv \frac{h_{j} \rho_{j} \Gamma_{j}^{2}}{\rho_{a}}
$$

The dynamics of the jet cocoon are largely determined by $\tilde{L}$, which is effectively the ratio of energy density in the jet and the ambient medium.

While the jet head continues forward, the high pressure cocoon also drives a shock into the ambient medium. Its speed is set by balacing the ambient ram pressure with the cocoon pressure, leading to $\beta_{c} \sim\left(p_{c} / \rho_{a} c^{2}\right)^{1 / 2}$ (Bromberg et al. 2011). As long as $\beta_{c}<1$, this speed is slightly less than the sound speed in the outer cocoon $\left(c_{s, a}=\sqrt{\gamma_{a d} p_{c} / \rho_{a} c^{2}}\right.$, where $\gamma_{a d}$ is the adiabatic index $)$, and much less than the sound speed in the relativistic inner cocoon $(c / \sqrt{3})$. Therefore, any pressure differences perpendicular to the jet axis are quickly smoothed out (Bromberg et al. 2011). At the same time, the relativistic sound speed in the inner cocoon establishes causal contact along most of the length of the jet. Consequently, the pressure within the cocoon is uniform to a good approximation as long as $p_{c} \ll \rho_{a} c^{2}$ (Bromberg et al. 
2011).

Many authors have contributed to advancing our analytical understanding of the physics of jet propagation. Begelman \& Cioffi (1989) considered the confinement of a non-relativistic jet by an overpressured cocoon and applied the results to interpret radio jets. Mészáros \& Waxman (2001) studied the propagation of a GRB jet through a red supergiant progenitor, but focused mainly on the jet head and not the cocoon. Matzner (2003) extended tthe Begelman \& Cioffi (1989) model to include relativistic jets. He assumed a conical jet of constant opening angle, thereby ignoring the effects of collimation. Lazzati \& Begelman (2005) and Morsony et al. (2007) attempted to include collimation, but because they did not properly treat dissipation by the collimation shock, they ended up with a collimation shock that did not converge to the axis. Komissarov \& Falle (1997) and Bromberg \& Levinson (2009) more carefully considered the geometry of the collimation shock and found that it indeed converges. Most recently, Bromberg et al. (2011) has put all of these pieces together to develop a fully self-consistent model for jet propagation, both in the collimated and uncollimated regimes.

Because the Bromberg et al. (2011) model is relevant to many problems we encounter in this thesis, we briefly overview some of their key results. They take $L_{j}$, $\theta_{0}$, and $\rho_{a}$ as given, and calculate the other dynamical variables. Their solution is applicable for $\Gamma_{0}>\theta_{0}^{-1}$, where $\Gamma_{0}$ is the Lorentz factor of the unshocked jet, and for as long as material continues to flow into the jet head. As discussed above, the dynamics are set by $\tilde{L}$; Bromberg et al. (2011) find that this parameter can be expressed as:

$$
\tilde{L}=\max \left[\left(\frac{L_{j}}{\rho_{a} t^{2} \theta_{0}^{4} c^{5}}\right)^{2 / 5},\left(\frac{L_{j}}{\rho_{a} t^{2} \theta_{0}^{2} c^{5}}\right)\right]
$$

$\tilde{L}$ takes on the first value when the jet is collimated, and the second when it is 
uncollimated. The dynamics can then be broken into the following five regimes, depending on the value of $\tilde{L}$ :

1. The collimated, non-relativistic regime $(\tilde{L}<1)$ : The jet is collimated and the head is non-relativistic, with $\beta_{h}=\tilde{L}^{1 / 2}$. The cocoon pressure is approximately uniform, and the cocoon speed is $\beta_{c} \simeq \theta_{0} \beta_{h}$. The Lorentz factor in the shocked jet is given by $\Gamma_{1} \sim \theta_{0}^{-1}$.

2. The collimated, relativistic regime $\left(1<\tilde{L}<\theta_{0}^{-4 / 3}\right)$ : The head of the jet becomes mildly relativistic, with $1 \lesssim \Gamma_{h} \lesssim \theta_{0}^{-1 / 3}$, but the cocoon pressure is still sufficient to collimate the jet. The cocoon pressure is still constant and the above expressions for $\beta_{c}$ and $\Gamma_{1}$ still apply.

3. The uncollimated, causal regime $\left(\theta_{0}^{-4 / 3}<\tilde{L}<4 \theta_{0}^{-4}\right)$ : The pressure in the cocoon is no longer sufficient to confine the jet, and the flow becomes uncollimated. However, the head is still slow enough that different parts of it are in causal contact. Therefore, all of the jet energy is still able to flow into the cocoon. The cocoon speed may become mildly relativistic $\left(\theta_{0}^{1 / 3} \lesssim \beta_{c} \lesssim 1\right)$, so that the uniform pressure approximation begins to break down.

4. The uncollimated, noncausal regime $\left(4 \theta_{0}^{-4}<\tilde{L} 4 \Gamma_{0}^{4}\right)$ : The head Lorentz factor becomes greater than $\theta_{0}^{-1}$, and as a result the head loses causal contact in the transverse direction. Energy begins to build up in the jet head; the energy in the jet head soon exceeds that in the cocoon. The cocoon speed becomes relativistic, and the approximation of uniform pressure no longer applies. Since $\beta_{h} \sim \beta_{c} \sim 1$, the cocoon takes on a roughly spherical shape.

5. The free expansion regime $\left(4 \Gamma_{0}^{4}<\tilde{L}\right)$ : The Lorentz factor of the head approaches $\Gamma_{h} \approx \Gamma_{0}$. The reverse shock becomes weak, and the ambient medium 
has a negligible effect on the jet's propagation.

For a standard GRB occurring in a massive progenitor star surrounded by a typical circumstellar medium, the jet head is non-relativistic while it drills through the star (Matzner 2003), corresponding to regime 1 above. Then after it breaks out of the star, the jet goes straight to the free expansion phase, regime 5 (Bromberg et al. 2011). For this reason, comparatively little effort has been put into understanding the intermediate dynamical regimes (2, 3, and 4). However, recent observations and theoretical work have turned up evidence that many SN progenitors, including Type Ic SNe associated with GRBs, may be surrounded by an extended low-mass envelope (Nakar \& Piro 2014; Nakar 2015; Drout et al. 2016; Nicholl \& Smartt 2016; Taddia et al. 2016, see also Chapter 2). In this case regimes 2, 3 and 4 may all be encountered, depending on the jet and envelope properties. Understanding how this envelope affects the propagation of the jet, and how it alters emission from the GRB and its associated SN, is a major theme of this work.

In addition to the analytical models mentioned above, several authors have conducted numerical studies of jet propagation in the progenitor star. Zhang et al. (2003) found that the jet converts much of its energy to internal energy while traversing the star (as expected for a collimated jet). This leads to acceleration and expansion of the jet after breakout as internal energy is reconverted to kinetic energy. Mizuta et al. (2006) studied how the propagation depends on injection Lorentz factor. Morsony et al. (2007) performed the first GRB jet simulations with an adaptive mesh, and investigated the transition of the jet from collimated to uncollimated after it breaks out of the star. Mizuta \& Aloy (2009) explored how various progenitor star models affect jet propagation, finding a correlation between the angular energy distribution in the post-breakout jet and the mass of the star. The effect of the jet timescale 
on the subsequent explosion was studied by Lazzati et al. (2012). Mizuta \& Ioka (2013) looked at how the jet opening angle and Lorentz factor after breakout correlate with the jet injection conditions, and also numerically calibrated the Bromberg et al. (2011) model in the non-relativistic regime. The propagation of GRB jets in the interstellar medium has also been investigated, e.g. by Zhang \& MacFadyen (2009), Wygoda et al. (2011), and van Eerten \& MacFadyen (2012). Other researchers have performed simulations of extragalactic jets (Marti et al. 1995; Martí et al. 1997; Aloy et al. 1999; Hughes et al. 2002).

\subsection{Radiation from relativistic jets I: The prompt phase}

Observationally, GRB behavior is conveniently separated into a prompt phase when the initial burst of X-rays and $\gamma$-rays is emitted, and an afterglow phase with a lower flux and longer timescale. The conversion of the kinetic energy of the relativistic ejecta into radiation is a complicated process, and even today the prompt radiation mechanism of GRBs remains mysterious. Here, we briefly overview prompt observations of GRBs and qualitatively discuss several possibilities for the production of the prompt high-energy emission. We discuss the afterglow in the next section.

The observed spectrum of GRBs is nonthermal, and can generally be described by a Band function, which is basically two power-laws smoothly connected by an exponential (Band et al. 1993). While the Band model typically gives an excellent fit to the spectrum, it is strictly phenomenological and tells us nothing about the underlying physics. The spectrum peaks at energy $E_{p}$. The peak energy tends to vary smoothly in time, and there is a well-known correlation, now known as the Amati 
correlation, between the peak energy and the isotropic energy of the burst $E_{\text {iso }}$ (Amati et al. 2002). The burst timescale is most commonly measured using $T_{90}$, which is the time after which $90 \%$ of the GRB photons have arrived. The low energy radiation in GRBs is delayed relative to the high energy emission, a phenomenon known as spectral lag. The amount of lag correlates inversely with the burst luminosity (Norris et al. 2000).

The light curve of most GRBs displays a high degree of variability on timescales much shorter than $T_{90}$. The short variability timescale constrains the size of the emitting region, providing evidence that GRBs originate from compact objects. Related to this is the compactness problem, which is essentially a discrepancy between the observation of a nonthermal spectrum (which implies an optically thin source), and the extremely high optical depth $\tau_{\gamma \gamma}$ for pair creation obtained from a naive (i.e., non-relativistic) calculation. When relativistic sources are considered, the pair creation optical depth is smaller, and the compactness problem is resolved as long as the Lorentz factor is sufficiently high (Piran 2004). The typical minimum Lorentz factor required is $\gtrsim 100$ for most bursts. We note, however, that not all bursts show high variability. In particular, long-duration LLGRBs such as GRB 060218 instead have a smooth lightcurve (Campana et al. 2006), and this has ramifications for LLGRB modeling, as we discuss in Chapter 2. GRB 060218 also has a much lower minimum Lorentz factor ( $\gtrsim 2$ ) than is typical (Toma et al. 2007).

In order to produce prompt radiation, kinetic energy in the jet must somehow be dissipated and converted to internal energy. The most common way to accomplish this is through shocks, although relativistic turbulence (e.g., Smolsky \& Usov 2000) or electromagnetic dissipation (Usov 1994; Lyutikov \& Blandford 2004; Thompson 2006) are also possibilties. The shocks can be either external shocks (e.g., the forward 
and reverse shock that form at the interface of the jet and the external medium), or internal shocks within the jet itself, which arise for example if the central engine emits material of varying Lorentz factor, so that faster ejecta collide with previously emitted slower ejecta. Both internal shocks (Narayan et al. 1992; Rees \& Meszaros 1994) and external shocks (Rees \& Mészáros 1992) have been explored as the power source of prompt GRB emission. The physical mechanism responsible for the prompt emission in these shock models is typically synchrotron radiation from electrons accelerated by the shocks. Generally speaking, external shock models have trouble producing variability on timescales much shorter than the burst timescale, while in internal shock models this feature arises naturally (Sari \& Piran 1997). Internal shocks are therefore the more popular choice for standard long GRBs. On the other hand, in bursts that do not show much variability, internal shock models are less plausible, as we discuss in Section 2.4.7.

Recently, a new picture for GRB prompt emission has started to emerge, spurred on by recent observations which have revealed a significant quasi-thermal spectral component (in addition to the usual Band function component) in a number of bursts. A review by Pe'er (2015) provides a list of GRBs for which thermal emission has been claimed. Axelsson \& Borgonovo (2015) have also shown that the spectral width of many bursts is too wide to be explained by synchrotron emission, suggesting a different origin for the prompt emission. The similar timescale of the thermal and nonthermal emission motivates consideration of a model where the nonthermal emission is produced by Comptonization of the thermal photons. In this "photospheric model," magnetic fields, internal shocks, or some other mechanism dissipate some of the bulk kinetic energy of a GRB jet into leptons that then upscatter thermal radiation from the jet photosphere. As shown by Chhotray \& Lazzati (2015) and 
Pe'er (2015), when the optical depth at which dissipation occurs is low, the result is a spectrum consisting of a Comptonized blackbody component plus a nonthermal power-law tail. Ryde (2005) demonstrated that many GRB spectra are well-fit by this thermal component + power-law tail model. Detailed numerical modeling of the photospheric emission in relativistic jets has been carried out by several authors, and the theoretical light curves and spectra are qualitatively similar to typical GRB light curves (Lazzati et al. 2009, 2011, 2013; Mizuta et al. 2011; Ito et al. 2013). Moreover, the photospheric model has promise in explaining several correlations between common GRB observables (Fan et al. 2012; Lazzati et al. 2013). For further discussion of the photospheric model, see Sections 2.4.1 and 2.4.7.

\subsection{Radiation from relativistic jets II: The after- glow phase}

Well after the prompt $\gamma$-ray emission has faded away, GRBs remain visible in other wavelengths due to continued interaction of the external shocks with the circumstellar medium. Emission during this "afterglow" phase tends to be smoothly varying, and to evolve from higher to lower frequency. Afterglow detections were first made at X-ray wavelengths by Costa et al. (1997), at optical wavelengths by van Paradijs et al. (1997), and at radio frequencies by Frail et al. (1997). Since then, many more afterglows have been uncovered by Swift, with fruitful results. Optical afterglow measurements led to the first direct measurement of redshift in a GRB, confirming that GRBs occur at cosmological distances (Metzger et al. 1997). Meanwhile, radio afterglow observations provided direct evidence that the ejecta producing the afterglow is relativistic (e.g., Frail et al. 1997). Some so-called "dark" bursts are not detected in 
the optical, possibly due to high optical extinction along the line of sight (Greiner et al. 2011).

Afterglow spectra tend to be a broken power-law. The light curves in some cases are simple power-laws in time, and in other cases show more structure, with an early plateau followed by a steep decay. The specific flux at a given frequency is commonly expressed by $F_{\nu} \propto t^{-\alpha} \nu^{-\beta}$; Piro (2001) find $\alpha \sim 1.4$ and $\beta \sim 0.9$ at X-ray wavelengths for a large sample of bursts.

The interaction of relativistic ejecta produced by the central engine with an external density produces a thin shell of shocked gas that plows into the circumstellar environment. During the initial phase of the afterglow, this shell coasts with an approximately constant Lorentz factor $\gamma \sim \Gamma_{0}$ (Sari \& Piran 1995), corresponding to the free expansion regime discussed in Section 1.2. Eventually, once the shell has swept up a sufficient mass, it begins to decelerate. The deceleration becomes important when the mass swept up by the shell is equal to its rest mass over $\gamma$ (Rees \& Mészáros 1992). Later, once the shell has swept a mass equal to its rest mass, the outflow becomes non-relativistic (Sari \& Piran 1995).

Blandford \& McKee (1976) studied the self-similar evolution of a relativistic blast wave sweeping up an external medium, which is applicable to the decelerating, relativistic phase of the dynamics. In a constant density medium, the Lorentz factor of this blast wave evolves with radius as $\gamma \propto R^{-3 / 2}$. Due to light travel time effects, the time $t$ that elapses in the observer frame and the time $t_{l a b}$ that elapses in the lab frame are related by $t \approx t_{l a b} / 2 \gamma^{2}$. Therefore $R \approx 2 \gamma^{2} c t$, and the radius and $\gamma$ evolve with observer time as

$$
R \propto t^{-1 / 4}
$$


and

$$
\gamma \propto t^{-3 / 8}
$$

Sari \& Piran (1995) and Sari (1997) discussed the evolution of the forward and reverse shocks in the coasting, decelerating, and non-relativistic limits. The dynamics are completely characterized by two dimensionless parameters: $\gamma$, and $f$, the ratio of the comoving upstream density at the reverse shock to that at the forward shock.

The above analysis assumes a constant density medium, and a constant Lorentz factor of the ejecta inwards of the reverse shock. It can be generalized to the case where the circumstellar environment is a stellar wind (e.g., Chevalier \& Li 1999, 2000), or to the case where the ejecta have a distribution of Lorentz factors, with more mass at slower speeds (e.g., Rees \& Mészáros 1998; Sari \& Mészáros 2000). Both cases lead to a slower deceleration of the forward shock, in the former because less mass is swept as the radius increases, and in the latter because slower ejecta continually catch up with and "refresh" the shocked shell. In Section 2.4.7 and Appendix A, we also generalize to the case where there is continued energy input from a long-lived central engine. In that case, the forward shock may actually accelerate, if the outer density profile is sufficiently steep.

Although the outflow is initially beamed, as long as $\gamma>\theta_{0}^{-1}$ it appears indistinguishable from a spherical blast wave to an on-axis observer, due to relativistic beaming. Modeling the afterglow radiation during this time can provide an estimate of $E_{i s o}$, the isotropic equivalent energy of the burst. As the shell decelerates and $\gamma$ drops below $\theta_{0}^{-1}$, a steep drop in the observed light curve, known as the "jet break," is expected for two reasons. First, the jet edge comes into view, resulting in a lower observed flux than for a spherical blast wave. Second, the flow undergoes a dynamical transion at $\gamma \sim \theta_{0}^{-1}$ and begins to expand sideways. Measuring the time of the jet 
break allows a direct determination of $\theta_{0}$. Combining this with an estimate of $E_{\text {iso }}$ allows for a determination of the true energy in the burst, $E=E_{\text {iso }} \theta_{0}^{2} / 2$. Observations of long GRBs show remarkable consistency in $E$, with a typical value $10^{51} \mathrm{ergs}$ and a spread of only a factor of $\sim 2$ (Piran 2004).

The process of jet spreading, i.e. the transition from beamed to quasi-spherical flow, has been investigated by many authors, both analytically (Rhoads 1999; Sari et al. 1999; Livio \& Waxman 2000; Chevalier \& Li 2000; Wygoda et al. 2011) and numerically (Zhang \& MacFadyen 2009; Wygoda et al. 2011; van Eerten \& MacFadyen 2012, 2013). We discuss this topic in more detail in Section 2.4.5, as it is relevant to our model of GRB 060218.

The standard afterglow radiation model involves synchrotron emission from the external shocks, and was developed by Sari et al. (1996), Mészáros \& Rees (1997), and Sari et al. (1998). In the observer frame, the power $P\left(\gamma_{e}\right)$ and frequency $\nu\left(\gamma_{e}\right)$ of an electron moving with Lorentz factor $\gamma_{e}$ in the frame of the shocked gas are (Rybicki \& Lightman 1979; Sari et al. 1998):

$$
P\left(\gamma_{e}\right)=\frac{4}{3} \sigma_{T} c \gamma^{2} \gamma_{e}^{2} \frac{B^{2}}{8 \pi}
$$

and

$$
\nu\left(\gamma_{e}\right)=\gamma \gamma_{e}^{2} \frac{q_{e} B}{2 \pi m_{e} c},
$$

where $\sigma_{T}$ is the Thomson cross section, $m_{e}$ is the electron mass, $q_{e}$ is the electron charge, and $B$ is the comoving magnetic field. In the observer frame, the time for an electron with $\gamma_{e}$ to cool is $t_{c}=6 \pi m_{e} c / \sigma_{T} \gamma B^{2} \gamma_{e}$ (Sari et al. 1998). Setting $t_{c}$ equal to the age $t$ determines a characteristic Lorentz factor $\gamma_{c}$, such that electrons with $\gamma_{e}>\gamma_{c}$ cool in time $t$, and those with $\gamma_{e}<\gamma_{c}$ do not. 
Electrons the shocked region are typically assumed to be accelerated by a Fermi mechanism to a power-law distribution in Lorentz factor with index $p$, i.e. $N(E) \propto$ $\gamma_{e}^{-p}$, with some minimum Lorentz factor $\gamma_{m}$. A further assumption is that fixed fractions $\epsilon_{e}$ and $\epsilon_{B}$ of the postshock energy density end up in nonthermal relativistic electrons and magnetic fields, respectively. $\epsilon_{e}$ is assumed to be large enough that $\gamma_{m} \gg 1$. Then, the $\epsilon$-parameters are related to the other quantities by (Sari et al. 1998):

$$
\epsilon_{e}=\frac{\gamma_{m}}{\gamma} \frac{p-1}{p-2} \frac{m_{e}}{m_{p}}
$$

and

$$
\epsilon_{B}=\frac{B^{2}}{32 \pi \gamma^{2} c^{2} n m_{p}}
$$

where $n$ is the number density of the external medium, and $m_{p}$ is the proton mass.

The net afterglow spectrum is obtained by integrating the single-electron synchrotron spectrum over the distribution of $\gamma_{e}$. The result is different depending on whether $\gamma_{m}>\gamma_{c}$ (all electrons can cool), or $\gamma_{m}<\gamma_{c}$ (only some electrons can cool). These regimes are known as fast-cooling and slow-cooling, respectively. Defining characteristic frequencies $\nu_{m}=\nu\left(\gamma_{m}\right)$ and $\nu_{c}=\nu\left(\gamma_{c}\right)$, the spectral flux is (Sari et al. 1998)

$$
F_{\nu}=\left\{\begin{array}{lc}
\left(\frac{\nu}{\nu_{c}}\right)^{1 / 3} F_{\nu, \max }, & \nu<\nu_{c} \\
\left(\frac{\nu}{\nu_{c}}\right)^{-1 / 2} F_{\nu, \max }, & \nu_{c}<\nu<\nu_{m} \\
\left(\frac{\nu_{m}}{\nu_{c}}\right)^{-1 / 2}\left(\frac{\nu}{\nu_{m}}\right)^{-p / 2} F_{\nu, \max }, & \nu>\nu_{m}
\end{array}\right.
$$


in the fast cooling case, and

$$
F_{\nu}=\left\{\begin{array}{lc}
\left(\frac{\nu}{\nu_{m}}\right)^{1 / 3} F_{\nu, \max }, & \nu<\nu_{m} \\
\left(\frac{\nu}{\nu_{m}}\right)^{(1-p) / 2} F_{\nu, \max }, & \nu_{m}<\nu<\nu_{c} \\
\left(\frac{\nu_{c}}{\nu_{m}}\right)^{(1-p) / 2}\left(\frac{\nu}{\nu_{c}}\right)^{-p / 2} F_{\nu, \max }, & \nu>\nu_{c}
\end{array}\right.
$$

in the slow cooling case, where the maximum spectral flux is given by

$$
F_{\nu, \max }=\frac{R^{3} n m_{e} \gamma B}{9 D^{2} q_{e}}
$$

$R$ is the radius of the shocked shell, and $D$ is the distance to the source.

By combining this model for the radiation with the dynamical models discussed above, the full evolution of the afterglow spectrum and light curve can be accounted for. A caveat is that at low (typically radio) frequencies, synchrotron self-absorption becomes important, which introduces another characteristic frequency, $\nu_{a}$. The spectrum and light curve then depend on the relative ordering of $\nu_{a}, \nu_{c}$, and $\nu_{m}$, as discussed by various authors (Katz 1994; Katz \& Piran 1997; Granot et al. 2000; Granot \& Sari 2002). Here we have assumed a constant density medium for simplicity, but the model has been extended to a wind-like medium as well (Chevalier \& Li 1999, 2000).

The standard synchrotron picture describes GRB afterglows remarkably well given its simplicity, and constrains many physical properties of the burst. The index $p$ can be found by measuring the high-energy spectral slope, with typical values of $p \simeq 2.4$ (Panaitescu \& Kumar 2002). If $p$ is known, measuring the slope of the light curve also gives the power-law index of the external density profile. Some bursts are better described by a constant density medium, and others are better described by a wind- 
like medium (Chevalier \& Li 1999). Finally, if $\epsilon_{e}$ and $\epsilon_{B}$ are assumed, then $E_{i s o}$ and $n$ can be estimated. We apply the synchrotron afterglow model to describe the radio emission of GRB 060218 in Section 2.4.5, but we find that the late X-rays in that burst require a different explanation (see Section 2.4.4). While here we have focused on synchrotron emission, it is also possible for the external shocks to produce highenergy radiation via inverse Compton scattering, if there is a photon source at smaller radii (e.g., due to dissipation in the jet). We discuss this further in Section 2.4.1, Section 2.4.7, and Appendix B. 
Chapter 2

Jet or Shock Breakout? The Low-Luminosity GRB 060218 


\begin{abstract}
We consider a model for the low-luminosity gamma-ray burst GRB 060218 that plausibly accounts for multiwavelength observations to day 20. The model components are: (1) a long-lived $\left(t_{j} \sim 3000 \mathrm{~s}\right)$ central engine and accompanying lowluminosity $\left(L_{j} \sim 10^{47} \mathrm{erg} \mathrm{s}^{-1}\right)$, mildly relativistic $(\gamma \sim 10)$ jet; $(2)$ a low-mass $\left(\sim 4 \times 10^{-3} M_{\odot}\right)$ envelope surrounding the progenitor star; and (3) a modest amount of dust $\left(A_{V} \sim 0.1 \mathrm{mag}\right)$ in the circumstellar or interstellar environment. Blackbody emission from the transparency radius in a low-power jet outflow can fit the prompt thermal X-ray emission, and the nonthermal X-rays and $\gamma$-rays may be produced via Compton scattering of thermal photons from hot leptons in the jet interior or the external shocks. The later mildly relativistic phase of this outflow can produce the radio emission via synchrotron radiation from the forward shock. Meanwhile, interaction of the associated SN 2006aj with a circumstellar envelope extending to $\sim 10^{13} \mathrm{~cm}$ can explain the early optical emission. The X-ray afterglow can be interpreted as a light echo of the prompt emission from dust at $\sim 30$ pc. Our model is a plausible alternative to that of Nakar, who recently proposed shock breakout of a jet smothered by an extended envelope as the source of prompt emission. Both our results and Nakar's suggest that bursts such as GRB 060218 may originate from unusual progenitors with extended circumstellar envelopes, and that a jet is necessary to decouple the prompt emission from the supernova.
\end{abstract}

\title{
$2.1 \quad$ Introduction
}

Low-luminosity gamma-ray bursts (LLGRBs) are a subclass of long-duration GRBs that, although rarely detected and not yet well understood, have the potential to shed light on more commonly observed cosmological bursts. Though the uncertainty 
is large, estimated volumetric rates indicate that LLGRBs occur some 10 - 100 times more often than typical GRBs (Soderberg et al. 2006), making them a compelling population for further study. In addition, LLGRBs take place nearby, so the associated supernovae ( $\mathrm{SNe}$ ) can be detected easily and studied in detail, placing constraints on energetics and circumstellar environment and giving clues about the SN-GRB connection. Phenomena like central engine activity, jet-star and jet-wind interactions, and the transition from beamed to spherical outflow can be probed more thoroughly than is possible at high redshift, and any insight into the radiation mechanisms of LLGRBs can inform our understanding of the GRB population at large.

Among known LLGRB sources, the remarkably similar sources GRB 060218/SN 2006aj (Campana et al. 2006; Soderberg et al. 2006; Mazzali et al. 2006; Kaneko et al. 2007; Pian et al. 2006) and GRB 100316D/SN 2010bh (Starling et al. 2011; Chornock et al. 2010; Fan et al. 2011; Cano et al. 2011; Margutti et al. 2013) stand out as unique due to their long timescale, smooth single-peaked light curve, anomalously soft and bright X-ray afterglow, and the presence of significant thermal X-ray and optical components at early times (Campana et al. 2006; Kaneko et al. 2007; Starling et al. 2011; Margutti et al. 2013). Several important and compelling questions concerning these two bursts remain open. In a narrow sense, the atypical prompt emission, the origin of the X-ray blackbody component, and the unusual X-ray afterglow are hard to explain in terms of standard GRB theory. In a broader sense, we do not know whether the progenitor system is the same as for typical GRBs: do these ultra-long LLGRBs represent the low-luminosity end of a continuum of collapsar explosions, or a different stellar endpoint altogether? The answer to this question has important implications for high-mass stellar evolution, the connection between SNe and GRBs, and the low-energy limits of GRB physics, especially considering that events similar to GRB 
060218 and GRB 100316D are likely more common than cosmological GRBs. The peculiar nature of these bursts, the wealth of timely observations in many wavebands (especially for GRB 060218), and the lack of a consensus picture for their behavior make these particular LLGRBs prime targets for theory.

Accordingly, a wide variety of models have been proposed to explain the many facets of GRB 060218. Campana et al. (2006) and Waxman et al. (2007) originally modeled the prompt X-ray emission as shock breakout from a circumstellar shell at $\sim 10^{12} \mathrm{~cm}$. The breakout duration in this case, assuming spherical symmetry, is only a few hundred seconds; however, Waxman et al. (2007) suggested asphericity as a means to lengthen the burst timescale. On the other hand, Ghisellini et al. (2007b) argued against the shock breakout interpretation, showing that fine tuning is required to bring about a large change in breakout timescale through asymmetrical effects. Ghisellini et al. (2007a) presented an alternative synchrotron self-absorption model for the prompt emission, but the high brightness temperature and small emitting area in their model are at odds with radio observations, which suggest only mildly relativistic speeds (Soderberg et al. 2006). Dai et al. (2006) found that Compton scattering of soft input photons off relativistic external shocks driven by an inner outflow could roughly reproduce the observed prompt light curve. In the same vein, Wang et al. (2007) showed that a Fermi acceleration mechanism could upscatter breakout thermal photons, creating a high energy power law tail to the thermal distribution. However, it is unlikely that thermal equilibrium is obtained in a relativistic breakout, and photon energies are limited by Compton losses (Katz et al. 2010; Nakar \& Sari 2010, 2012). Li (2007) and Chevalier \& Fransson (2008) investigated the prompt UV/optical emission, and demonstrated that shock breakout could reproduce the optical flux, given a large breakout radius of $5 \times 10^{13} \mathrm{~cm}$. (This large radius was initially viewed as a problem; 
see, however, the discussion in Section 2.3 below.) Björnsson (2008) also put forth a model for the prompt UV, based on optically thick cyclotron emission. Nakar \& Piro (2014) showed that an early UV/optical peak could be attributed to cooling emission from an extended low-mass circumstellar envelope shock-heated by the passage of fast SN ejecta. They did not discuss the case of SN 2006aj, although it appears in their Figure 1 as an example of extended envelope interaction.

Another possibility for the prompt emission is that GRB 060218 is an ordinary GRB jet viewed off-axis. However, a solely geometrical effect should result in a frequency-independent, or achromatic, break in the light curve, whereas the break in GRB 060218 is chromatic in nature (Amati et al. 2006). Mandal \& Eichler (2010) considered a scenario for GRB 060218 in which primary radiation scatters off material radiatively accelerated slightly off-axis from the line of sight; this acceleration can explain the chromatic behavior of the afterglow. However, as their model still required an unusually soft, long-duration, and low-luminosity primary photon source, it did not give insight into the fundamental factor distinguishing LLGRBs from most bursts.

Soderberg et al. (2006) and Fan et al. (2006) tackled the X-ray and radio afterglow. In each case, the radio could be explained by a synchrotron self-absorption in a wide $(\theta \gtrsim 1)$, mildly relativistic $(\Gamma \sim 2-3)$ outflow, but the high $\mathrm{X}$-ray afterglow flux could not be accounted for in a simple external shock synchrotron model. Soderberg et al. (2006) attributed this X-ray excess to a forming magnetar, while Fan et al. (2006) preferred late-time fallback accretion on to a central compact object. Toma et al. (2007) suggested that the radio afterglow was better explained by the late nonrelativistic phase of an initially collimated jet outflow. They inferred a jet luminosity $10^{45} \mathrm{erg} \mathrm{s}^{-1}$, an initial jet Lorentz factor $\Gamma \sim 5$, and an initial jet opening angle $\theta \sim 0.3$, and showed that a hot low-luminosity jet could successfully penetrate a 
WR star and expand upon breakout to achieve these initial conditions. Based on the smooth light curve and long engine duration, they posited a neutron star-powered rather than black hole-powered central engine. Barniol Duran et al. (2015) calculated the synchrotron afterglow light curves from a relativistic shock breakout, and while their model could fit the radio emission of GRB 060218, it predicted too low a flux and too shallow a temporal decay for the X-ray afterglow. Margutti et al. (2015) analyzed the X-ray afterglows of 12 nearby GRBs and established that GRB 060218 and GRB 100316D belong to a distinct subgroup marked by long duration, soft-photon index, and high absorption. They proposed the possibility that these afterglows are in fact dust echoes from shells tens of parsecs across that form at the interface between the progenitor's stellar wind and the ISM.

Until recently, most existing models have focused on explaining a particular aspect of this burst (e.g., the prompt nonthermal emission, the radio afterglow, or the optical emission), while leaving the other components to speculation. Nakar (2015) recently suggested a model that attempts to unify the prompt X-rays, early optical peak, and radio emission. In his picture, the prompt X-ray and optical emission arise from the interaction of a typical GRB jet with a low-mass envelope surrounding the progenitor star. The short-lived jet is able to tunnel through the progenitor star, but is choked in the envelope, powering a quasi-spherical, mildly relativistic explosion akin to a low-mass SN. The prompt X-rays are produced by the shock breaking out of the optically thick envelope (as described in Nakar \& Sari 2012), and optical radiation is emitted as the envelope expands and cools (as in Nakar \& Piro 2014). Interaction of the breakout ejecta with circumstellar material (CSM) generates the radio via synchrotron radiation (as in Barniol Duran et al. 2015). Nakar's model does not, however, attempt to explain the unusual X-ray afterglow or the presence of thermal 
X-rays at early times.

In this paper, we present a plausible alternative to Nakar's model for this peculiar burst, building on previous jet models. In Section 2.2, we give an overview of observations of GRB 060218, and discuss the key features that must be reproduced by any model. In Section 2.3, we address some problems with a straightforward shock breakout view for the prompt emission, and provide motivation for adopting a longlived jet instead. In Section 2.4, we describe how each component of the observed radiation is generated in our engine-driven model for GRB 060218, and check that our picture is self-consistent. Advantages, drawbacks, and predictions of our model, ramifications for GRB classification, and future prospects are discussed in Section 2.5, before we conclude in Section 2.6.

\subsection{Overview of observations}

The X-ray evolution of GRB 060218 and GRB 100316D can be divided into a prompt phase, an exponential or steep power-law decline, and an afterglow phase. Remarkably, these two objects share many observational features, perhaps suggesting that they have similar origins. In both objects, we see:

- Prompt nonthermal X-rays and $\gamma$-rays with a Band-like spectrum, but with lower luminosity, lower peak energy, and longer timescale as compared to cosmological GRBs.

- Thermal X-rays with roughly constant temperature $\sim 0.1 \mathrm{keV}$ over the first $\sim 1000 \mathrm{~s}$.

- Strong thermal UV/optical emission on a timescale of hours to days.

- A radio afterglow lasting tens of days and implying mildly relativistic outflow. 
- An X-ray afterglow that is brighter and softer than expected in standard synchrotron models.

Any unified model for these bursts must account for each of these components. Here we summarise multiwavelength observations during the prompt and afterglow phases of GRB 060218 and GRB 100316D.

Prompt X-rays/ $\gamma$-rays: The nonthermal spectrum of GRB 060218 from $t=$ $200 \mathrm{~s}$ to $t=3000 \mathrm{~s}$ is well fit by a Band function (Band et al. 1993) with low-energy photon index $\Gamma_{1}=-1.0$ and high-energy photon index $\Gamma_{2}=-2.5$, implying $F_{\nu} \propto \nu^{0}$ at low energies and $F_{\nu} \propto \nu^{-1.5}$ at high energies. $\Gamma_{1}$ and $\Gamma_{2}$ remain roughly constant over the evolution (Toma et al. 2007). Kaneko et al. (2007) found a somewhat different low-energy index, $\Gamma_{1}=-1.4$, when fitting the spectrum with a cut-off power law instead of a Band function. These values are typical for long GRBs (Ghirlanda et al. 2007). The peak energy $E_{p}$ of the best-fitting Band function decreases as $E_{p} \propto t^{-1.6}$ from $t=600 \mathrm{~s}$ until the end of the prompt phase. At $700 \mathrm{~s}, E_{p}=10 \mathrm{keV}$ (Toma et al. 2007). Despite its low luminosity, GRB 060218 obeys the Amati correlation between $E_{p}$ and luminosity (Amati et al. 2006). In addition to the nonthermal Band function, a significant soft quasi-thermal component is present in the prompt spectrum (Campana et al. 2006; Kaneko et al. 2007; Friis \& Watson 2013). Campana et al. (2006) found that the blackbody temperature remains nearly constant at $0.17 \mathrm{keV}$ throughout the prompt phase (Campana et al. 2006). A later analysis by Kaneko et al. (2007) determined a slightly lower temperature, $0.14 \mathrm{keV}$, for times after several hundred seconds (see their Figure 7). Throughout the prompt phase, the implied blackbody radius of the thermal X-rays increases slowly as $t^{0.3}$, with a value of $\sim 10^{12} \mathrm{~cm}$ at $t=2000 \mathrm{~s}$ (Campana et al. 2006).

The prompt XRT (0.3-10 keV) light curve of GRB 060218 can be decomposed 
into contributions from the thermal and nonthermal parts; the nonthermal component dominates until approximately 3000 s (Campana et al. 2006; see also Figure 1 in Ghisellini et al. 2007b). The total (nonthermal + thermal) isotropic-equivalent luminosity in the XRT band grows as $L_{X R T} \propto t^{0.6}$ for the first $1000 \mathrm{~s}$, when it reaches a peak luminosity $\sim 3 \times 10^{46} \mathrm{erg} \mathrm{s}^{-1}$, then declines as roughly $t^{-1}$ until $\sim 3000 \mathrm{~s}$, fading exponentially or as a steep power law after that (Campana et al. 2006). The thermal component initially comprises about $\sim 1 / 6$ of the total XRT band luminosity, and its light curve evolves similarly: at first it increases as a power law, rising steadily as $L_{t h} \propto t^{0.66}$ (Liang et al. 2006) until it peaks at $1 \times 10^{46} \mathrm{erg} \mathrm{s}^{-1}$ at $t=3000 \mathrm{~s}$ (Campana et al. 2006). At that time, the thermal and nonthermal luminosities are about equal, but during the steep decline phase (3000-7000 s), the thermal component comes to dominate the luminosity, indicating that the nonthermal part must decline more steeply (Campana et al. 2006). The light curve in the BAT band (15-150 keV) is initially very similar to the XRT light curve, increasing as about $t^{0.8}$ with roughly the same luminosity. Though its maximum luminosity $\left(\sim 3 \times 10^{46} \mathrm{erg} \mathrm{s}^{-1}\right)$ is similar to the peak XRT flux, the BAT flux peaks earlier, at $t=400 \mathrm{~s}$. Furthermore, it decays faster after the peak, falling off as $L_{B A T} \propto t^{-2}$ from 400-3000s (Campana et al. 2006; Toma et al. 2007).

Evidence for a blackbody spectral component has also been claimed for GRB 100316D, with a similar constant temperature $k T=0.14 \mathrm{keV}$ (Starling et al. 2011). However, the presence of this thermal component has been called into question based on a large change in its statistical significance with the latest XRT calibration software (Margutti et al. 2013). The nonthermal spectrum of this burst is similar to that of GRB 060218: its peak energy has about the same magnitude and declines in a similar fashion, and its low-energy photon index is also nearly the same over the first $\sim 1000$ 
seconds (Starling et al. 2011, see their Figure 4).

Compared to GRB 060218, GRB 100316D is more luminous in the XRT band, with $L_{X R T} \sim 10^{48} \mathrm{erg} \mathrm{s}^{-1}$. In this case, the XRT light curve has nearly constant luminosity $\left(\propto t^{-0.13}\right)$ for the first $800 \mathrm{~s}$ (Starling et al. 2011). (For this burst, there are no X-ray data available from 800-30000s.) If the light curve is broken into blackbody and Band function components, the nonthermal flux strongly dominates over the thermal contribution, with $L_{X R T} / L_{t h} \sim 30$.

Optical photometry: From the first detection of GRB 060218 at a few hundred seconds, the UV/optical emission slowly rises to a peak, first in the UV at $31 \mathrm{ks}$, and then in the optical at $39 \mathrm{ks}$ (Campana et al. 2006). The light curves dipped to a minimum at around $\sim 200 \mathrm{ks}$, after which a second peak occurred around $800 \mathrm{ks}$, which can be attributed to the emergence of light from the supernova SN 2006aj. Like other GRB-supernovae, 2006aj is a broad-lined Type Ic (Pian et al. 2006), but its kinetic energy $E_{k} \approx 2 \times 10^{51} \mathrm{erg}$ is an order of magnitude smaller than usual (Mazzali et al. 2006).

GRB 100316D was not detected with UVOT (Starling et al. 2011). Its associated supernova, SN 2010bh, peaked at 10 days (Cano et al. 2011). While detailed optical data is not available for the earliest times, SN 2010bh does show an excess in the Bband at $t=0.5$ days (Cano et al. 2011), which is at least consistent with an early optical peak.

X-ray/radio afterglow: Once the prompt emission of GRB 060218 has faded, another component becomes visible in the XRT band at $10000 \mathrm{~s}$. This afterglow has luminosity $L_{a g}=3 \times 10^{43} \mathrm{erg} \mathrm{s}^{-1}$ when it first appears, and fades in proportion to $t^{-1.2}$ until at least $t=10^{6} \mathrm{~s}$. While this power law decay is typical for GRBs, the time-averaged X-ray spectral index $\left(\beta\right.$ in $\left.F_{\nu} \propto \nu^{\beta}\right)$ is unusually steep, $\beta_{X}=-2.2$ 
(Campana et al. 2006; Soderberg et al. 2006). The measured spectral index at late times (0.5-10 days) is $\beta_{X}=-4.5$ (Margutti et al. 2015), suggesting that the spectrum softens over time.

Radio observations of GRB 060218 beginning around $\sim 1$ day indicate a power-law decay in the radio light curve with spectral flux $F_{\nu} \propto t^{-0.85}$ (Soderberg et al. 2006), not so different from the X-ray temporal decay and typical for GRBs. At 5 days, the spectrum peaked at the self-absorption frequency $\nu_{a} \approx 3 \mathrm{GHz}$ Soderberg et al. (2006). The radio to X-ray spectral index is unusually flat, $\beta_{R X}=-0.5$ (Soderberg et al. 2006). No jet break is apparent in the radio data (Soderberg et al. 2006), and self-absorption arguments indicate mildly relativistic motion (see Section 2.4.5).

The X-ray afterglow light curve of GRB 100316D can also be described by a simple power-law decay: $L_{a g} \propto t^{-0.87}$ from $t=0.4-10$ days, with X-ray luminosity $\sim 10^{43} \mathrm{erg} \mathrm{s}^{-1}$ at $t=0.4$ days. Like GRB 060218, its X-ray spectrum is also very soft, with $\beta_{X}=-2.5$ over the period 0.5-10 days (Margutti et al. 2015). Because of the gap in coverage, it is unclear precisely when the prompt phase ends and the afterglow phase begins.

GRB 100316D was detected at $5.4 \mathrm{GHz}$ from 11-70 days, with a peak at that frequency at $t \approx 30$ days (Margutti et al. 2013). This peak comes much later than that of GRB 060218, where the $5 \mathrm{GHz}$ peak occurred at 2-3 days (Soderberg et al. 2006). The late-time radio to $\mathrm{X}$-ray spectral index is $\beta_{R X}<-0.4$, comparable to GRB 060218 (Margutti et al. 2013). No jet break is detected out to 66 days, and the estimated Lorentz factor is again mildly relativistic, $\Gamma \sim 1.5-2$ on day 1 (Margutti et al. 2013). 


\subsection{Shock breakout or central engine?}

The majority of models for the prompt X-rays of GRB 060218 fall into two categories: shock breakout (e.g., Campana et al. 2006; Waxman et al. 2007; Nakar \& Sari 2012; Nakar 2015) or IC scattering of blackbody radiation by external shocks (e.g., Dai et al. 2006; Wang et al. 2007). The latter type requires seed thermal photons for IC upscattering; while Dai et al. (2006) and Wang et al. (2007) assumed these photons were produced by a shock breakout event, other thermal sources such as a dissipative jet are also possible. Here, we point out some difficulties with a shock breakout interpretation of the prompt X-ray emission, and suggest some reasons to consider a long-lived central engine scenario instead.

Early models for GRB 060218 (Campana et al. 2006; Waxman et al. 2007) considered the case where matter and radiation are in thermal equilibrium behind the shock, and the thermal X-rays and thermal UV/optical emission arise from shell interaction and shock breakout, respectively. However, for sufficiently fast shocks the radiation immediately downstream of the shock is out of thermal equilibrium, so the breakout temperature can be higher than when equilibrium is assumed (Katz et al. 2010; Nakar \& Sari 2010). In this scenario the prompt emission peaks in X-rays and the prompt spectrum is a broken power law with $F_{\nu} \propto \nu^{0}$ at low energies and $F_{\nu} \propto \nu^{-1.74}$ at high energies (Nakar \& Sari 2012). This is similar to the Band function spectrum observed in GRB 060218, motivating consideration of the case where the nonthermal X-rays originate from a relativistic shock breakout while the thermal UV/optical component comes from a later equilibrium phase of the breakout, as described in Nakar \& Sari (2012). This interpretation still has possible problems. For one, because the breakout radius implied by this model $\left(\sim 5 \times 10^{13} \mathrm{~cm}\right)$ is large, the origin of a separate thermal $\mathrm{X}$-ray component with photospheric radius $\sim 10^{12} \mathrm{~cm}$ is unclear in this picture. In 
addition, the evolution of the prompt peak energy differs from the expectation for a relativistic shock breakout as in Nakar \& Sari (2012). In GRB 060218, the peak energy falls off as $t^{-1.6}$, while in the relativistic shock breakout model it declines more slowly as $t^{-(0.5-1)}$. Consequently, while the peak energy inferred from relativistic breakout, $\sim 40 \mathrm{keV}$, is consistent with observations at early times (less than a few hundred seconds), the Nakar \& Sari (2012) model overestimates $E_{p}$ for most of the prompt phase. Another problem is that the optical blackbody emission is observed from the earliest time in GRB 060218, and it rises smoothly in all UVOT bands until peak. In the nonequilibrium shock breakout scenario, thermal optical emission would not be expected until later times, when equilibrium has been attained. A final issue with the shock breakout picture of Nakar \& Sari (2012) is that it involved a stellar mass explosion. Since only a small fraction of the energy goes into relativistic material in a standard SN explosion, the energy required for the breakout to be relativistic was extreme, $E_{S N} \gtrsim 10^{53}$ ergs. This high energy is inconsistent with the unremarkable energy of the observed SN, $2 \times 10^{51}$ ergs.

One can also consider the case where the prompt optical emission is attributed to shock breakout, but the prompt X-rays have a different origin. The large initial radius in this case is incompatible with a bare WR star, and initially seemed to rule out a WR progenitor (Li 2007; Chevalier \& Fransson 2008). However, this calculation assumed that much of the stellar mass was located close to the breakout radius. An extended optically thick region containing a relatively small amount of mass could circumvent this difficulty. Such an envelope might be created by pre-explosion mass loss or a binary interaction. There is mounting evidence for the existence of such dense stellar environments around other transients such as SN Type IIn (Fransson et al. 2014, and references therein), SN Type IIb (Nakar \& Piro 2014), SN Type Ibn 
(e.g., Matheson et al. 2000; Pastorello et al. 2008; Gorbikov et al. 2014), and SN Type Ia-CSM (Silverman et al. 2013; Fox et al. 2015).

The model of Nakar (2015) builds on the relativistic shock breakout model of Nakar \& Sari (2012), while solving several of its problems. Nakar (2015) introduces a low-mass, optically thick envelope around a compact progenitor. In his model, the explosion powering the breakout is driven not by the $\mathrm{SN}$, but by a jet that tunnels out of the progenitor star and is choked in the envelope, powering a quasi-spherical explosion. Having a large optically thick region preserves the long shock breakout timescale, but in this case most of the mass is concentrated in a compact core. Since the envelope mass is much smaller than the star's mass, the energy required for a relativistic breakout is reduced as compared to the model of Nakar \& Sari (2012). This picture also provides a natural explanation for the optical blackbody component via cooling emission from the shocked envelope. Moreover, as the breakout in this case is aspherical and only mildly relativistic, and occurs from the edge of an envelope with unknown density structure, the results of Nakar \& Sari (2012) (which assumed spherical, relativistic flow and a specific density structure) are not fully adequate for describing GRB 060218. Proper treatment of these differences may help to resolve issues with the evolution of $E_{p}$. However, it remains unclear whether Nakar's model can account for the simultaneous observation of optical and X-ray emission at early times, and the problem with interpreting the small blackbody radius of the thermal X-ray component persists.

Given the possible difficulties with shock breakout, a different source for the prompt X-ray radiation should be considered. Bromberg et al. (2011) have shown that a central engine origin for certain LLGRBs is unlikely as their duration $\left(T_{90}\right)$ is short compared to the breakout time. However, due to their relatively long $T_{90}$, 
engine-driven models are not ruled out for GRB 060218 and GRB 100316D. Furthermore, as discussed in Section 2.2, the prompt X-ray/ $\gamma$-ray emission of GRB 060218 shares much in common with typical GRBs. As these similarities would be a peculiar coincidence in the shock breakout view, a collapsar jet origin for GRB 060218 is worth investigating. Motivated by this, we consider the case where the early optical emission is powered by interaction of the $\mathrm{SN}$ ejecta with a circumstellar envelope, but the prompt X-rays originate from a long-lived jet.

\subsection{A comprehensive model for GRB 060218}

A schematic of our model is presented in Figure 2.1. The essential physical ingredients are a long-lived jet, an extended low-mass circumstellar envelope, and a modest amount of dust at tens of parsecs, which are responsible for the prompt X-rays/radio afterglow, early optical, and X-ray afterglow, respectively. Below we consider the origin of each observed component in detail, and show that a reasonable match to observations can be obtained for appropriate choices of the progenitor, jet, and CSM properties.

\subsubsection{Prompt thermal emission}

The thermal X-ray component is a puzzling aspect of GRB 060218, and it is not unique in this regard. A recent review by Pe'er (2015) lists a number of typical GRBs for which a Band + blackbody model improves the spectral fit, which has been claimed as evidence for thermal emission. Burgess et al. (2014) have also found evidence for thermal radiation in several other bursts. In fact, Axelsson \& Borgonovo (2015) have recently suggested that most bursts must contain a broadened thermal component, because in the majority of observed bursts, the full width half maximum 


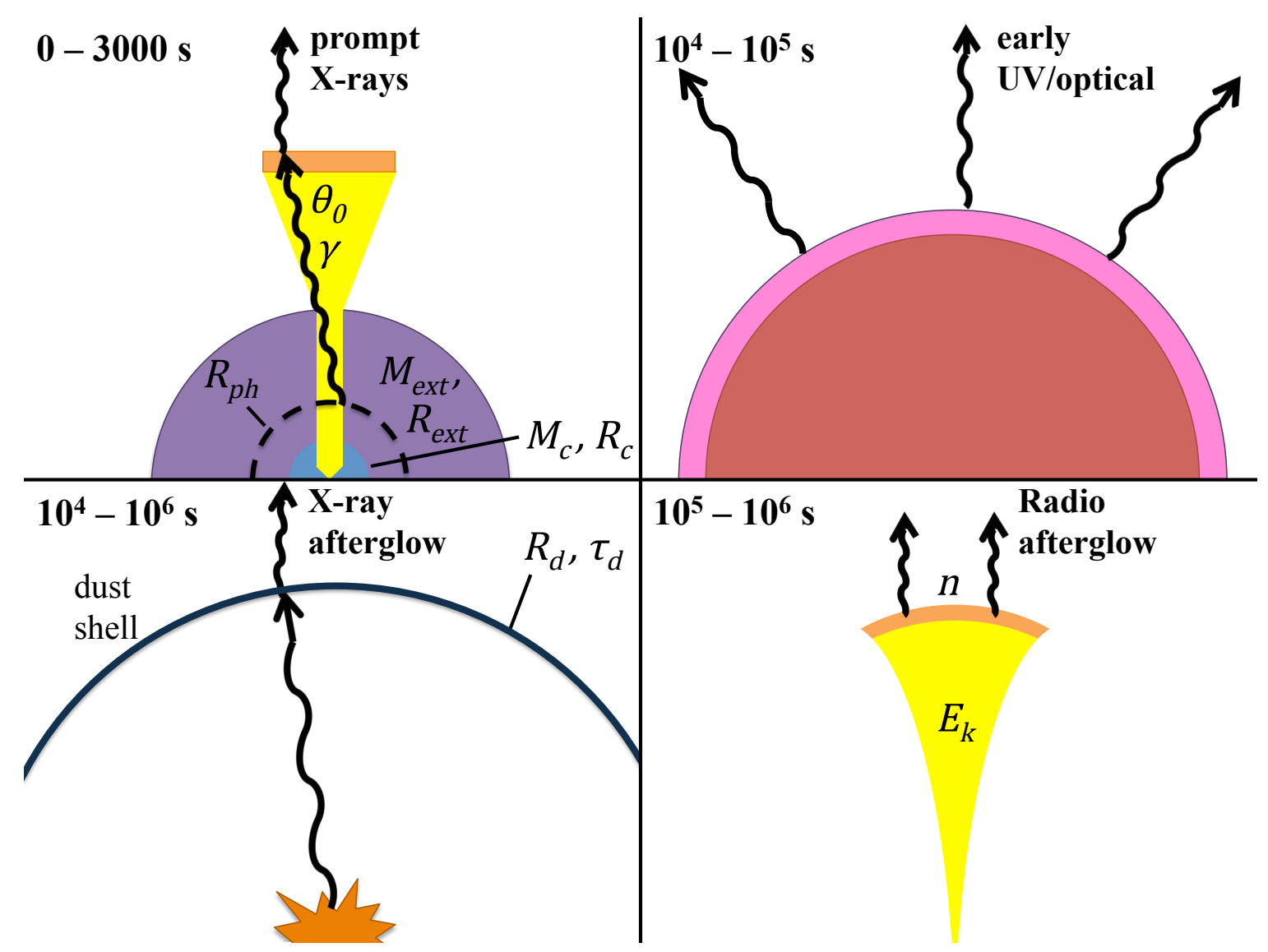

Fig. 2.1. - The origin of different components of the prompt and afterglow emission in our model. The figure is not to scale. The progenitor has a core-envelope structure. $M_{c} \sim 2 M_{\odot}$ is confined to a core of $R_{c} \sim 10^{11} \mathrm{~cm}$ (blue), while a mass $M_{\text {ext }} \ll M_{c}$ is contained mostly near the edge of an extended envelope with $R_{e x t} \gg R_{c}$ (purple). Upper left: A long-lived, dissipative jet tunnels through the progenitor system. Upon breakout, it emits blackbody radiation from radius $R_{p h}$. Some thermal photons IC scatter from external shocks (orange) or the jet interior (yellow) to create the Bandlike nonthermal component. The jet obtains terminal opening angle $\theta_{0}$ and Lorentz factor $\Gamma_{0}$ after breakout. Upper right: Fast SN ejecta shock the envelope, heating it. The slower bulk of SN ejecta (red) then lift the hot envelope (pink), which emits in the optical and UV as it expands and cools. Lower left: The prompt X-rays undergo scattering in a dusty region with inner radius $R_{d} \sim$ tens of pc and X-ray scattering optical depth $\tau_{d}$. The resulting light echo outshines the synchrotron afterglow, giving rise to a characteristic soft spectrum. Lower right: External shock synchrotron emission from the mildly relativistic phase of the jet generates the radio afterglow. 
of the spectral peak is narrower than is physically possible for synchrotron radiation. Although prompt thermal radiation is observationally indicated, the physical origin of this emission is yet unclear. One possible source of thermal X-rays is a jet-blown cocoon, although the flat early light curve of GRB 060218 and GRB 100316D is hard to explain in this case (Pe'er et al. 06; Starling et al. 2012; see also Ramirez-Ruiz et al. 2002). Another possibility is that the blackbody emission is produced at the transparency radius in a dissipative jet outflow, as discussed in the context of GRB 060218 by Ghisellini et al. (2007a) and Ghisellini et al. (2007b). Here we consider the latter scenario.

Paczynski (1990), who considered photospheric emission from super-Eddington neutron star winds, showed that the photospheric radius in a spherical mildly relativistic outflow is $R_{p h}=\left(\dot{M}_{i s o} \kappa / 4 \pi \tau_{p h} \beta c\right)(1-\beta)$, where $\dot{M}_{i s o}$ is the isotropic mass loss rate, $c$ is the speed of light, $\beta$ is the the wind velocity in units of $c, \kappa$ is the opacity, and $\tau_{p h} \approx 1$ is the optical depth at the photosphere. Let $L_{t h}$ and $T_{0}$ be the observerframe luminosity and temperature, and define the Lorentz factor $\gamma=\left(1-\beta^{2}\right)^{-1 / 2}$. Then the comoving luminosity and temperature are $\bar{L}_{t h}=\left(1+\beta^{2}\right)^{-1} \gamma^{-2} L_{t h}$ and $\bar{T}_{0}=\gamma^{-1} T_{0}$. (Here and below, a bar indicates that a quantity is measured in the frame comoving with the engine-driven outflow.) Substituting $\bar{L}_{t h}, \bar{T}_{0}$, and $R_{p h}$ into the Stefan-Boltzmann equation, one may derive $\dot{M}_{i s o}$ in terms of the observables $L_{t h}$ and $T_{0}$ :

$$
\dot{M}_{i s o}=\frac{c \tau_{p h}}{\kappa}\left(\frac{4 \pi L_{t h}}{\sigma_{B} T_{0}^{4}}\right)^{1 / 2} \frac{\beta \gamma}{(1-\beta)\left(1+\beta^{2}\right)^{1 / 2}}
$$

where $\sigma_{B}$ is the Stefan-Boltzmann constant.

Based on observations of the thermal component in GRB 060218, we take the luminosity to evolve as a power law, $L_{t h}=L_{0}\left(t / t_{L}\right)^{k}$, before some time $t_{L}$, and to decline exponentially as $L_{t h}=L_{0} e^{\left(t_{L}-t\right) / t_{\text {fold }}}$ after $t_{L}$. An empirical fit to the data 
of Campana et al. (2006) gives $L_{0} \approx 10^{46} \mathrm{erg} \mathrm{s}^{-1}, t_{L} \approx 2800 \mathrm{~s}$, and $t_{\text {fold }} \approx 1140 \mathrm{~s}$. Liang et al. (2006) found $k \approx 0.66$ by fitting the thermal component in the $0.3-2 \mathrm{keV}$ band. We set the temperature to a constant, $T_{0}$, and define $\xi=T_{0} /(0.17 \mathrm{keV})$. For simplicity, we assume the outflow is injected at a constant Lorentz factor; this is supported by the near constant observed temperature, as otherwise the comoving temperature would have to vary in such a way to precisely cancel the change in $\gamma$. Scaling $L_{0}$ to $10^{46} \mathrm{erg} \mathrm{s}^{-1}$ and $\kappa$ to $0.2 \mathrm{~cm}^{2} \mathrm{~g}^{-1}$, and setting $\tau_{p h}=1$, the mass loss and kinetic luminosity $L_{i s o}=(\gamma-1) \dot{M}_{i s o} c^{2}$ prior to $t_{L}$ are

$$
\dot{M}_{i s o}(t)=1 \times 10^{-9} \kappa_{0.2}^{-1} L_{0,46}^{1 / 2} \xi^{-2}\left(t / t_{L}\right)^{k / 2} \beta \gamma^{3} M_{\odot} \mathrm{s}^{-1}
$$

and

$$
L_{\text {iso }}(t)=2 \times 10^{45} \kappa_{0.2}^{-1} L_{0,46}^{1 / 2} \xi^{-2}\left(t / t_{L}\right)^{k / 2} \beta \gamma^{3}(\gamma-1) \operatorname{erg~s}^{-1}
$$

We have assumed the Lorentz factor $\gamma$ of the outflow is large enough that the approximation $(1-\beta)^{-1}\left(1+\beta^{2}\right)^{-1 / 2} \approx \sqrt{2} \gamma^{2}$ applies; at worst, this differs from the exact expression by a factor $2^{1 / 2}$ when $\beta \rightarrow 0$. The isotropic mass and energy of the jet are then

$$
\begin{aligned}
M_{i s o} & =\int_{0}^{\infty} M_{i s o} d t \\
& =4 \times 10^{-6} \kappa_{0.2}^{-1} L_{0,46}^{1 / 2} \xi^{-2}\left(\frac{t_{\text {eng }}}{3100 \mathrm{~s}}\right) \beta \gamma^{3} M_{\odot}
\end{aligned}
$$

and

$$
\begin{aligned}
E_{\text {iso }} & =\int_{0}^{\infty} L_{\text {iso }} d t \\
& =7 \times 10^{48} \kappa_{0.2}^{-1} L_{0,46}^{1 / 2} \xi^{-2}\left(\frac{t_{\text {eng }}}{3100 \mathrm{~s}}\right) \beta \gamma^{3}(\gamma-1) \mathrm{ergs},
\end{aligned}
$$

where $t_{\text {eng }} \equiv 2 t_{L} /(2+k)+t_{\text {fold }}$. If the outflow is beamed into opposing jets with a small opening angle $\theta_{0}$, the true mass and energy of the ejected material are $M_{j}=$ $\left(\theta_{0}^{2} / 2\right) M_{i s o}$ and $E_{j}=\left(\theta_{0}^{2} / 2\right) E_{i s o}$. For a mildly relativistic flow $(\gamma \sim$ a few $)$ with 
$\theta_{0} \sim 10^{\circ}$, we have $M_{j} \sim 10^{-6} M_{\odot}$ and $E_{j} \sim 10^{49}$ ergs. The photosphere at $R_{p h} \approx 7 \times$ $10^{11} L_{0,46}^{1 / 2} \xi^{-2}\left(t / t_{L}\right)^{k / 2} \gamma \mathrm{cm}$ expands subrelativistically with average speed $\sim R_{p h} / t_{L} \sim$ $0.01 \gamma c$. Note that the photosphere lies within the radius of the low-mass envelope, $R_{\text {ext }} \simeq 9 \times 10^{12} \mathrm{~cm}$, that we derive in Section 2.4 .3 below, suggesting that dissipation occurs within the envelope. The time $t_{L}$ corresponds to the time when the central engine shuts off.

In the above calculation, we have assumed for simplicity that the jet outflow is directed into an uncollimated cone. However, as we show in Section 2.4.6, the jet may be collimated within the envelope, and become uncollimated only after breaking out. The decollimation timescale can be estimated as the time for the jet's cocoon to expand and become dynamically unimportant after breakout, which is $\sim R_{e x t} / c_{s} \sim$ $3^{1 / 2} R_{\text {ext }} / c \sim 500 \mathrm{~s}$, where $c_{s}$ is the sound speed. This is short compared to the duration of prompt emission; therefore, outside of the envelope, the assumption of an uncollimated outflow is reasonable for most of the prompt phase. However, it appears that the photosphere is within the envelope. The decollimation timescale there might be longer because it will take the jet some time to excavate the walls of the narrow hole left by its passage. Collimation has the joint effect of decreasing the outflow's opening angle (due to the confining effect of the cocoon) and decreasing its Lorentz factor (due to more of the total jet energy going into internal versus kinetic energy). Both of these effects lead to a smaller $M_{j}$ and $L_{j}$, for the same observed thermal luminosity and temperature. Thus, by ignoring collimation we potentially overestimate these quantities; our derived mass loss rate and kinetic luminosity should really be viewed as upper limits. 


\subsubsection{Extinction and absorption}

The optical/UV extinction and the X-ray absorption to GRB 060218 are crucial for the interpretation of observations of the event, as well as giving information on gas and dust along the line of sight. The early optical/UV emission is strongly weighted to the ultraviolet, which is especially sensitive to absorption. The amount of Galactic absorption is not controversial; extinction maps of the Galaxy yield $E(B-V)=$ $0.14 \mathrm{mag}$, while the Galactic Na I D lines indicate $E(B-V)=0.13$ mag (Guenther et al. 2006; Sollerman et al. 2006). The reddening has been estimated from the narrow $\mathrm{Na} I \mathrm{D}$ lines in the host galaxy as being $E(B-V)=0.042 \mathrm{mag}$, or $A_{V}=$ $0.13 \pm 0.01 \mathrm{mag}$ (Guenther et al. 2006). As noted by Sollerman et al. (2006), a larger reddening is possible if there is ionization in the host galaxy. However, the properties of the host galaxy derived from fitting the spectral energy distribution and the observed Balmer line decrement point to a low extinction so Sollerman et al. (2006) advocate the low value obtained from the Na I D line. Our model for the late-time X-rays (see Section 2.4.4) also suggests a similar low extinction.

A higher host galaxy reddening, $E(B-V)=0.2 \mathrm{mag}$, was advocated by Campana et al. (2006) and Waxman et al. (2007) because the early ( $<1$ day) emission could be fitted by a Rayleigh-Jeans spectrum, consistent with high temperature emission. This suggestion allowed a shock breakout model for both the thermal X-ray emission and the early optical emission. This value of the reddening was also used by Nakar (2015), who noted that the implied blackbody temperature is $>50,000 \mathrm{~K}$. Nakar (2015) advocates the large reddening based on the slow color evolution leading up to the peak, which is expected in the Rayleigh-Jeans limit. However, his model could in principle accommodate a smaller extinction, if the model is consistent with a constant temperature leading to the peak. 
In view of the lack of direct evidence for the larger values of extinction in the host, we take the small value that is directly indicated. Thöne et al. (2011) had derived some of the observed parameters for GRB 060218 based on Galactic extinction only. As expected, the spectrum is then not well approximated by a Rayleigh-Jeans spectrum and a temperature in the range 30,000-35,000 K is deduced over the first half day. A blackbody fit gives the radius at the time of peak luminosity, $10^{14} \mathrm{~cm}$, which yields a luminosity of $5 \times 10^{43} \mathrm{erg} \mathrm{s}^{-1}$. This can be compared to the luminosity $>3 \times 10^{44} \mathrm{erg} \mathrm{s}^{-1}$ found by Nakar (2015) in his larger extinction model.

The X-ray absorption column density has been obtained by fitting the observed spectrum to a model with a power-law continuum, a blackbody thermal component and interstellar absorption; Kaneko et al. (2007) obtain an absorbing hydrogen column density of $N_{H}=6 \times 10^{21} \mathrm{~cm}^{-2}$ over 10 spectra covering the time of peak luminosity. Margutti et al. (2015) infer the same absorption column from fitting an absorbed power law to the afterglow spectra. There is no evidence for evolution of $N_{H}$. Using a standard conversion of $N_{H}$ to $A_{V}$ for the Galaxy, $N_{H}=2 \times 10^{21} A_{V} \mathrm{~cm}^{-2}$ (e.g., Güver \& Özel 2009), the corresponding value of $A_{V}$ is 3 . There is a significant difference between the extinction determined from the $\mathrm{Na}$ I line and that from the X-ray absorption.

One way to reconcile the difference is to have the dust be evaporated in the X-ray absorbing region. Waxman \& Draine (2000) have discussed evaporation of dust by the radiation from a GRB; optical/UV photons with energies $1-7 \mathrm{eV}$ are responsible for the evaporation. A normal burst with an optical/UV luminosity of $L_{\text {opt }}=1 \times 10^{49} \mathrm{erg} \mathrm{s}^{-1}$ can evaporate dust out to a radius of $R_{d} \simeq 10 \mathrm{pc}$ (Waxman $\&$ Draine 2000). Since $R_{d} \propto L_{\text {opt }}^{1 / 2}$ and the peak luminosity of GRB 060218 was about $1 \times 10^{43} \mathrm{erg} \mathrm{s}^{-1}$, we have $R_{d} \approx 0.01 \mathrm{pc}$ and the absorbing gas is likely to be 
circumstellar in origin.

\subsubsection{UV/optical emission}

Here we investigate the possibility that the optical emission is from shocked gas, but the X-ray emission is not. We take a supernova energy of $2 \times 10^{51} \mathrm{ergs}$ and a core mass of $2 M_{\odot}$, as determined from modeling the supernova emission (Mazzali et al. 2006b). The optical emission has a timescale of $\sim 1$ day, which is characteristic of supernovae thought to show the shock breakout phenomenon (see Fig. 10 in Modjaz et al. 2009), but the emission is brighter than that observed in more normal supernovae. As discussed in Section 2.3, there is increasing evidence that massive stars can undergo dense mass loss before a supernova. We thus consider the possibility that an extended, low-mass circumstellar medium is responsible for the high luminosity.

Nakar \& Piro (2014) have discussed how the shock breakout process is affected by the mass of an extended envelope. When most of the stellar mass is at the radius of the surrounding envelope, a standard shock breakout, as in Chevalier \& Fransson (2008), is expected. This case applies to SN 1987A (Chevalier 1992). However, when the envelope mass is much less than the core mass, the early emission is determined by the emission from the envelope that is heated by the expansion of the outer part of the core. One of the distinguishing features of the non-standard case is that the red luminosity can drop with time, which is not the case for standard shock breakout. Nakar \& Piro (2014) note in their Fig. 1 that the early emission from GRB 060218 shows a drop in the $\mathrm{V}$ emission that implies the non-standard, low-mass envelope case. Another difference is that in the standard case, the initially rising light curves turn over because the blackbody peak passes through the wavelength range of interest as the emission region cools (e.g., Chevalier \& Fransson 2008), while in the non-standard 
case the turnover is due to all the radiative energy in the envelope being radiated and the temperature remains steady (Nakar \& Piro 2014). The set of Swift-UVOT light curves in fact show approximately constant colors (and thus temperatures) through the luminosity peak at $\sim 3.5 \times 10^{4} \mathrm{~s}$ (Fig. 2 of Campana et al. 2006). The UVOT observations of GRB 060218 give the best set of observations of a supernova during this early non-standard phase.

Nakar (2015) has recently discussed the early emission from GRB 060218 in terms of interaction with a low mass envelope. The mass of the envelope was estimated at $0.01 M_{\odot}$ based on the timescale of the optical peak and an estimate of the shell velocity. However, the expansion of the envelope was attributed to an explosion driven by the deposition of energy from an internal jet. In this case, the event is essentially a very low mass supernova. In our model, the expansion is driven by the outer, high velocity gas of the supernova explosion, as in the non-standard expansion case of Nakar \& Piro (2014). The input parameters are a supernova explosion energy $E_{S N}=2 \times 10^{51} \mathrm{ergs}$ and core mass $M_{c}=2 M_{\odot}$ (Mazzali et al. 2006), a peak luminosity of $L_{p}=5 \times 10^{43} \mathrm{erg} \mathrm{s}^{-1}$ (Campana et al. 2006; Thöne et al. 2011), and a time of peak of $t_{p}=3.5 \times 10^{4} \mathrm{~s}$ (Campana et al. 2006). Since SN 2006aj was of Type Ic (no Helium or Hydrogen lines), we assumed an opacity $\kappa=0.2 \mathrm{~cm}^{2} \mathrm{~g}^{-1}$, appropriate for an ionized heavy element gas. These parameters can then be used to find the properties of the low mass extended envelope (subscript ext): $M_{\text {ext }} \approx 4 \times 10^{-3} M_{\odot}$, shell velocity $v_{\text {ext }} \approx 3 \times 10^{9} \mathrm{~cm} \mathrm{~s}^{-1}$, and energy $E_{\text {ext }} \approx 3 \times 10^{49}$ ergs. These results come from the dynamics of the outer supernova layers sweeping up and out the low mass envelope around the star, and the time of the peak luminosity (Nakar \& Piro 2014). The value of $R_{e x t} \approx 9 \times 10^{12} \mathrm{~cm}$ is proportional to luminosity, because of adiabatic expansion. The radius is related to the luminosity and thus the assumed absorption. These 
results are not sensitive to the density distribution in the extended envelope provided that most of the envelope mass is near $R_{\text {ext }}$. The mass in the envelope derived here is sufficient that the shock wave breaks out in the envelope, as assumed in the model. At the time of maximum luminosity, the radius of the shell is $R_{p} \approx v_{\text {ext }} t_{p}=1 \times 10^{14} \mathrm{~cm}$. As noted by Nakar \& Piro (2014), the minimum luminosity between the two peaks of the light curve can give an upper limit to the initial radius of the core. In the case of GRB 060218, the drop in the luminosity between the peaks is shallow so that only a weak limit on the core radius can be set, $R_{c} \lesssim 2 \times 10^{12} \mathrm{~cm}$.

These considerations show that the overall properties of the early optical/UV emission from GRB 060218 can be accounted for by a model in which there is shock breakout in a low mass, extended envelope. The model makes further predictions that can be tested in the case of GRB 060218. Approximating the observed temperature at the peak as the effective temperature leads to $T_{o b s} \approx 3.5 \times 10^{4} \mathrm{~K}$, which is consistent with the observed temperature of GRB 060218 at an age of 0.085-0.5 days (SI Fig. 17 of Thöne et al. 2011). The high temperature justifies the neglect of recombination in the model. Nakar \& Piro (2014) note that the optical depth of $M_{\text {ext }}$ becomes unity at $t \approx t_{p}\left(c / v_{e x t}\right)^{1 / 2}$, which is day 1.3 for GRB 060218; the photospheric velocity at this time gives an estimate for $v_{\text {ext }}$. The earliest spectrum of Pian et al. (2006) is on day 2.89 , when they estimate a photospheric velocity of $26,000 \mathrm{~km} \mathrm{~s}^{-1}$. The photospheric velocity is higher at earlier times, so there is rough agreement of the model with observations.

While the model reproduces the basic observational features, it is not clear whether it can reproduce the full evolution of the optical light curve and spectrum. This problem is non-trivial and hinges on details of the cooling envelope model, e.g. the mass-velocity distribution of ejecta, that are not yet well understood; we do not 
discuss this issue further.

\subsubsection{X-ray afterglow}

After a steep drop, the X-ray emission from GRB 060218 enters an apparent afterglow phase at an age of $0.1-10$ days. During this time, the flux spectrum is approximately a power law and the evolution is a power law in time: $F_{\nu} \propto \nu^{\beta_{X}} t^{-1.1}$ (Soderberg et al. 2006). Continuous spectral softening is observed, with $\beta_{X}$ decreasing from -2.2 at 0.1 day to $\sim-4.5$ at $\sim 3$ days. The time evolution is typical of a GRB afterglow, but the spectrum is unusually steep and the indices do not obey the standard "closure" relations for GRB afterglows (Fan et al. 2006). In view of this, other proposals have been made for this emission, e.g., late power from a central magnetar. Fan et al. (2006) considered a wide, accretion-powered outflow as the afterglow source, but the expected light curve in that case is $F_{\nu} \propto t^{-5 / 3}$, which seems too steep to explain the observations.

In standard GRB afterglow emission, there is one population of relativistic particles that gives rise to the emission, from radio to X-ray wavelengths. However, in GRB 060218, it is difficult to join the radio spectrum with the X-rays (see Fig. 1 in Soderberg et al. 2006); a flattening of the spectrum above radio frequencies would be necessary, as well as a sharp steepening at X-ray energies. In fact, some young supernova remnants such as RCW 86 show such spectra (Vink et al. 2006). The steepening would require some loss process for the high energy particles; however, Soderberg et al. (2006) find that synchrotron losses set in at a relatively low energy, so the observed spectrum cannot be reproduced. In addition, the X-ray evolution does not show a jet

break, as might be expected if the afterglow is produced in the external shocks of a collimated outflow. Barniol Duran et al. (2015) examined a shock breakout afterglow 
model for the late radio and X-ray emission. They were able to model the radio emission quite adequately, but the predicted X-ray emission was considerably below that observed, decayed too slowly in time, and had the incorrect spectral index. They concluded that the X-ray emission had some other source.

An alternative model for the emission was suggested by Shao et al. (2008), that it is a dust echo of emission close to maximum light. The light curve shape expected for an $\mathrm{X}$-ray echo is a plateau followed by evolution to a $t^{-2}$ time dependence. The observed light curve for GRB 060218 is between these cases, which specifies the distance of the scattering dust in front of the source, $\sim 50$ pc (Shao et al. 2008). Shao et al. (2008) applied the echo model widely to GRB light curves. However, Shen et al. (2009) noted two problems with this model for typical bursts. First, the required value of $A_{V}$ is typically $\sim 10$, substantially larger than that deduced by other means. Second, the evolution is generally accompanied by a strong softening of the spectrum that is not observed.

The case of GRB 060218 is different from the standard cases; it had a long initial burst and a large ratio of early flux to late flux. These properties are more favorable for echo emission. The early flux was $F_{p r} \approx 1 \times 10^{-8} \mathrm{erg} \mathrm{cm}^{-2} \mathrm{~s}^{-1}$ lasting for $t_{p r} \sim 2000 \mathrm{~s}$, while the late flux of $F_{\text {late }} \approx 1 \times 10^{-11} \mathrm{erg} \mathrm{cm}^{-2} \mathrm{~s}^{-1}$ lasted for $t_{\text {late }} \sim 20,000 \mathrm{~s}$. If the late emission is produced as an echo, the optical depth of the dust region is $\tau_{0}=F_{\text {late }} t_{\text {late }} / F_{p r} t_{p r}=0.01$ (Shen et al. 2009). The corresponding value of $A_{V}$ is $0.01-0.1$ (Shen et al. 2009). This value of $A_{V}$ is roughly consistent with that determined from the Na I D line, giving support to the echo interpretation.

To better understand the spectral softening and determine the dust properties, we numerically investigated the expected dust echo emission from a dust shell at radius $R_{d}$. We used the theory of Shao et al. (2008), with some modifications to specify to 
GRB 060218. While Shao et al. (2008) assumed a flat prompt spectrum in the range $0.3-10 \mathrm{keV}$ as is typical for cosmological GRBs, we instead used an empirical model including a blackbody as described in Section 2.4.1 and a Band function with flux and peak energy evolving according to Toma et al. (2007). In particular, the inclusion of the thermal component - which dominates at low energies - results in a steeper echo spectrum than predicted by Shao et al. (2008).

The parameters of the model are the dust radius $R_{d}$, the scattering optical depth at $1 \mathrm{keV} \tau_{\mathrm{keV}}$, the minimum and maximum grain sizes $a_{-}$and $a_{+}$, and the power-law indices $s$ and $q$ that set how the scattering optical depth per unit grain size scales with energy and grain radius, i.e. $\tau_{a} \propto \tau_{\mathrm{keV}} E^{-s} a^{4-q}$ with $2<s<3$ and $3<q<4$ typically. The echo flux is integrated over the range $0.3-10 \mathrm{keV}$, appropriate for the Swift XRT band. The parameter $a_{-} \approx 0.005 \mu \mathrm{m}$ is based on observations of Galactic dust grains (Mathis et al. 1977). The prompt photons are approximated as being injected instantaneously at $t=0$.

We find a reasonably good fit to the light curve with a reduced chi-squared of 1.6 when $\tau_{\mathrm{keV}} \approx 0.007, R_{d} \approx 28 \mathrm{pc}, a_{+}=0.25 \mu \mathrm{m}, s=2$ and $q=4.0$, as shown by the dashed black line in Fig. 2.2. The same model can satisfactorily reproduce the spectral evolution at late times, as depicted in Fig. 2.3. The optical depth is well-determined and robust to changes in the other parameters. There is a strong degeneracy between $R_{d}$ and $a_{+}$because the afterglow flux depends only on the combination $R_{d} a_{+}^{-2}$; however, $a_{+}=0.25 \mu \mathrm{m}$ is roughly consistent with Galactic observations (Mathis et al. 1977; Predehl \& Schmitt 1995). Varying $s$ does not greatly affect the light curve, but $s \approx 2$ is preferred to match the spectral index at late times.

Increasing $q$ while appropriately decreasing $R_{d}$ results in a light curve with the same initial flux, but a slower late decline. Therefore, a somewhat improved fit can 
be obtained if values of $q>4$ are considered. Taking the same value as above for $a_{+}$, we find the best overall fit with a reduced chi-squared of 1.0 when $\tau_{\mathrm{keV}} \approx 0.009$, $R_{d} \approx 6.3 \mathrm{pc}, s=2$ and $q=4.76$. Fig. 2.2 and Fig. 2.3 show that the light curve and spectral evolution in this higher- $q$ model do not differ greatly from the lower- $q$ model discussed above; as expected, the main difference is a better fit to the few data points at the latest times. Despite the improved fit, we prefer the lower- $q$ model, as $q=4$ is more consistent with typical dust measurements and previous dust echo afterglow models. An important takeaway is that, depending on the unknown dust properties $a_{+}$and $q, R_{d}$ can vary by an order of magnitude or more without significantly affecting the light curve. We stress that without a good handle on the characteristics of the circumburst dust, the true value of $R_{d}$ remains highly uncertain.

The scattering depth $\tau_{\text {sca }}$ at energy $0.8 \mathrm{keV} \lesssim E \lesssim 10 \mathrm{keV}$ can be converted to an optical extinction via the relation $\tau_{s c a} / A_{V} \approx 0.15(E / 1 \mathrm{keV})^{-1.8}$ (Draine \& Bond 2004). For $\tau_{\text {sca }}=0.007$ at $1 \mathrm{keV}, A_{V} \approx 0.05$, roughly in line with $\mathrm{Na} \mathrm{I} \mathrm{D}$ line observations and the simple estimate above. (We note that it is not necessary for these values to coincide: as the typical scattering angle is $\alpha_{\text {sca }} \sim 0.1^{\circ}-1^{\circ}$, the line of sight to the afterglow and the prompt source are separated by $\sim \alpha_{\text {sca }} R_{d} \sim 0.1-1 \mathrm{pc}$. It is possible that the ISM properties could vary on this scale.) We conclude that a moderate amount of dust located between a few parsecs and several tens of parsecs from the progenitor can plausibly explain the anomalous X-ray afterglow.

Due to the gap in observations from 1000-30000s, the late X-ray light curve in GRB 100316D is difficult to model in detail. nonetheless, some simple estimates can be made. The prompt X-ray emission has luminosity $L_{p r} \sim 3 \times 10^{46} \mathrm{erg} \mathrm{s}^{-1}$ and timescale $t_{p r} \sim 1000 \mathrm{~s}$ (Margutti et al. 2013). The X-ray afterglow has luminosity $L_{\text {late }} \sim 2 \times 10^{43} \mathrm{erg} \mathrm{s}^{-1}$ at $t_{\text {late }} \sim 3 \times 10^{4} \mathrm{~s}$ and decays as $t^{-0.87}$ (Margutti et al. 2013), 


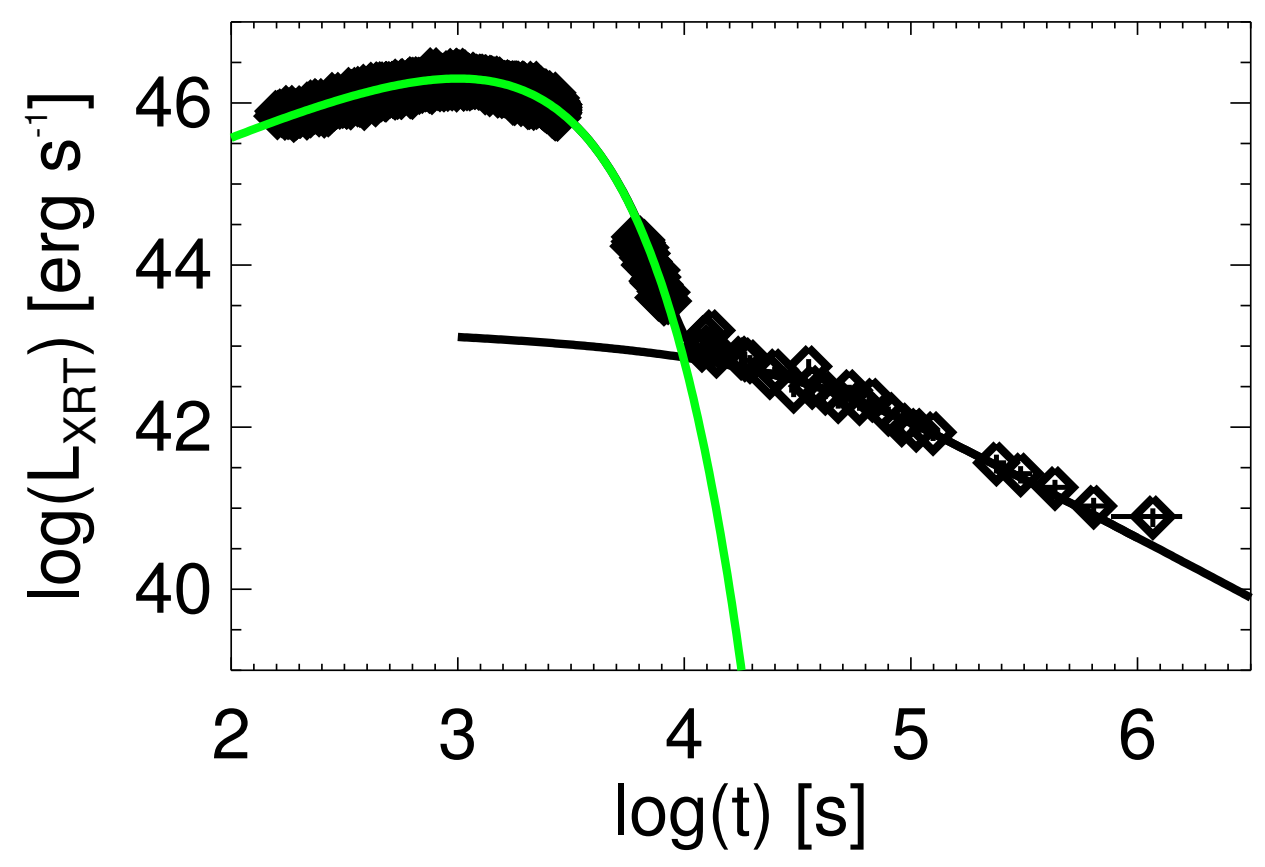

Fig. 2.2.- Prompt and afterglow light curves for the dust echo model. The prompt data points are fit with a simple exponentially cut-off power-law, shown by the green line. The solid and dashed black lines indicate the contribution from dust scattering at $R_{d}$. The model parameters are respectively $\tau_{\mathrm{keV}} \approx 0.009, R_{d} \approx 6.3 \mathrm{pc}$, and $q=4.76$ (solid line), and $\tau_{\mathrm{keV}} \approx 0.007, R_{d} \approx 28 \mathrm{pc}$, and $q=4.00$ (dashed line). Both echo models have $a_{+}=0.25 \mu \mathrm{m}, a_{-}=0.005 \mu \mathrm{m}$, and $s=2$.

so $L_{\text {late }} t_{\text {late }}$ gives a reasonable estimate of the reradiated energy. The above lead to a similar estimate for the optical depth as for GRB 060218, $\tau_{d} \sim 0.02$, or $A_{V} \sim 0.2$. One interesting difference between the two bursts is that the spectral index of the late afterglow, $\beta_{X}=-2.5$, is harder in GRB 100316D than in GRB 060218 where $\beta_{X}=-4.5$. (Notably, GRB 060218 is the only burst with such a steep afterglow spectrum; GRB 100316D is more typical, as other soft-afterglow bursts such as GRB 090417B and GRB 130925A also show $\beta_{X} \sim-2.5$ (Margutti et al. 2015).) In the echo interpretation, this discrepancy can be explained partially by a difference in 


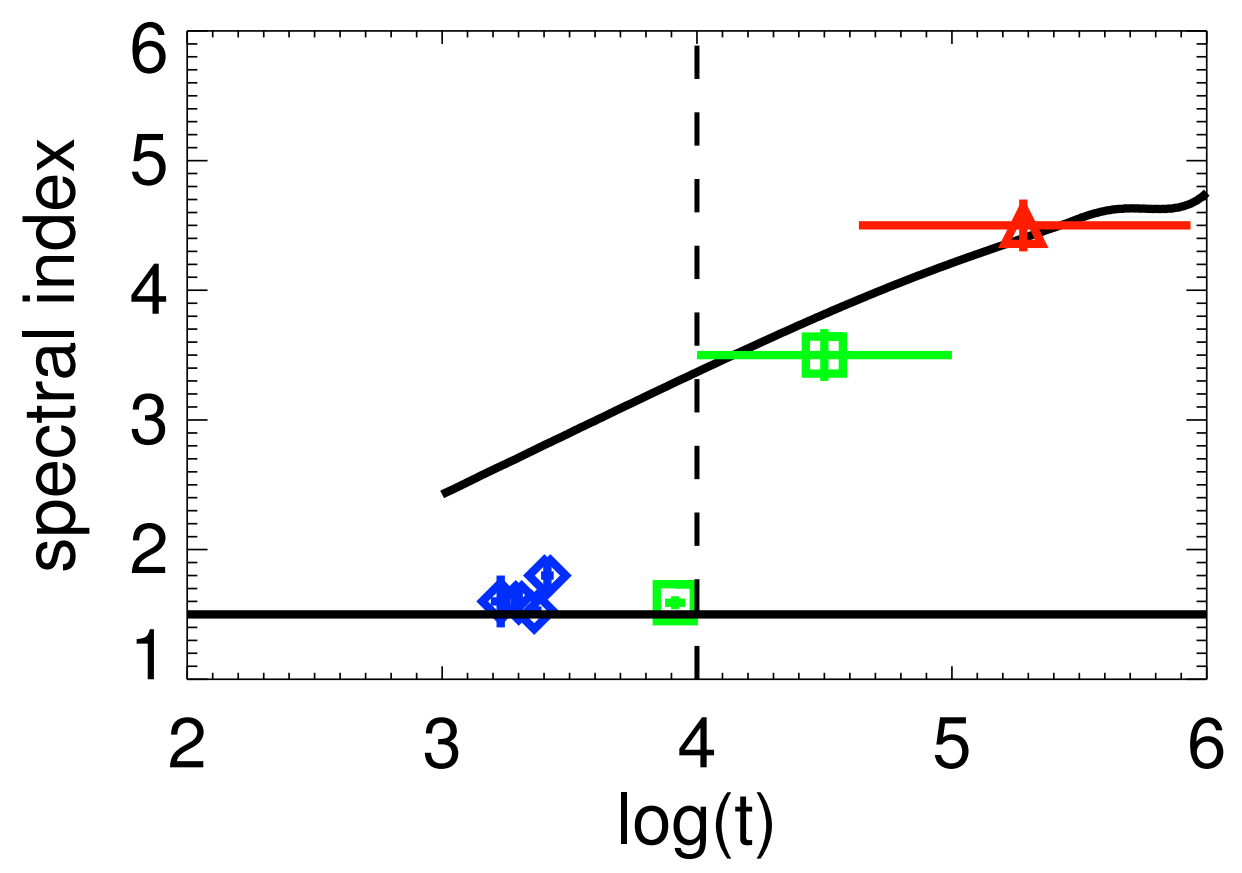

Fig. 2.3. - Spectral evolution in GRB 060218. The blue, green, and red points are taken from Table 1 in Toma et al. (2007), Figure 1 in Ghisellini et al. (2007a), and Margutti et al. (2015) respectively. The lower solid black line indicates the typical high-energy spectral index of the Band function, $F_{\nu} \propto \nu^{-1.5}$. The upper solid and dashed black lines shows the two-point XRT flux spectral index, $\log (F(10 \mathrm{keV}) / F(0.3 \mathrm{keV})) / \log (10 / 0.3)$, as a function of time for the same pair of models as in Fig. 2.2. The time when the echo flux first exceeds the prompt flux in our model is shown by the vertical dashed line.

the prompt spectrum. Due to the presence of a strong thermal component at low energies, the time-averaged prompt $0.3-10 \mathrm{keV}$ spectrum of GRB 060218 is steeper than in GRB 100316D, where the thermal component is weak and the spectrum is essentially flat at low energies. However, this effect alone is not sufficient, as it only produces a change in spectral index of $\sim 1$. $R_{d}$ and $a_{+}$also have a strong effect on $\beta_{X}$ because they change timescale for spectral steepening, as does the energy dependence of the scattering cross section. A larger $R_{d}$, smaller $a_{+}$, or lower value of $s$ (compared 
to our values for GRB 060218) may be necessary to obtain the correct $\beta_{X}$ in GRB 100316D. However, due to a lack of data regarding the time dependence of $\beta_{X}$ and an insufficient light curve, we cannot say which of these effects is the relevant one.

Margutti et al. (2015) have recently argued that four bursts, including GRB 060218 and GRB 100316D, belong to a distinct subclass of transient taking place in a complicated CSM. They base their claim on the unlikelihood of three unrelated properties - high absorption column, soft afterglow spectrum, and long duration - occurring together by chance. They invoke a wind-swept dusty shell to account for the high X-ray absorption and steep afterglow spectrum (through an echo of the prompt emission), and propose shock breakout in a complex local CSM to explain the long duration of prompt emission, preferring this interpretation to one in which the central engine duration is intrinsically long. Our findings support their suggestion that the very soft spectrum of GRB 060218 arises from a dust echo, but as the amount of dust in our model is not particularly high, an especially dense shell is not necessary; the dust could exist in an ISM of typical density and chemistry. We stress that the absorption column implied by dust extinction in our model is not consistent with the X-ray absorption column inferred from the prompt emission, as the latter is larger by a factor of $\sim 30$. For this reason, dust scattering and X-ray absorption are unlikely to be occurring in the same place in GRB 060218. Rather, the X-ray absorption is likely happening at small radii where dust has been evaporated. Also, while our results do indicate a dense envelope around the progenitor star, we also differ from the Margutti et al. (2015) picture by adopting an intrinsically long-lived central engine.

Our results can be compared to two other objects for which dust echo models have been proposed, GRB 130925A (Evans et al. 2014; Zhao \& Shao 2014) and GRB 090417B (Holland et al. 2010). The optical extinction inferred from modeling the 
afterglow as a dust echo is $A_{V}=7.7 \mathrm{mag}$ in GRB 130925A, (Evans et al. 2014), and in GRB 090417B it is $A_{V} \gtrsim 12 \mathrm{mag}$ (Holland et al. 2010). In each case, the amount of dust required to fit the X-ray afterglow via an echo is consistent with the absorbing hydrogen column needed to fit the X-ray spectrum (Evans et al. 2014; Holland et al. 2010). In GRB 090417B, the high extinction can also explain the lack of an optical detection (Holland et al. 2010). In contrast to GRB 060218 and GRB 100316D, GRB 130925A and GRB 090417B appear to have taken place in an unusually dusty environment, with the dust accounting for both the X-ray scattering afterglow and the large $N_{H}$.

Interestingly, these bursts also differ in their prompt emission. GRB 130925A appears typical of the ultra-long class of objects described by Levan et al. (2014), which also includes GRB 101225A, GRB 111209A, and GRB 121027A. Compared to GRB 060218 and GRB 100316D, these ultra-long bursts are more luminous and longer lived, and they show variability in their light curves on short timescales, reminiscent of typical GRBs (Levan et al. 2014). The light curve of GRB 090417B is qualitatively similar to GRB 130925A, and it likewise has a longer timescale, higher luminosity, and more variability compared to GRB 060218 (Holland et al. 2010). Thus, while Margutti et al. (2015) have made a strong case that GRB 060218, GRB 100316D, GRB 130925A, and GRB 090417B constitute a population distinct from cosmological LGRBs, upon closer inspection GRB 130925A and GRB 090417B differ strikingly from GRB 060218 and GRB 100316D. It seems, then, that three discrete subclasses are needed to explain their observations: 1) smooth light curve, very low-luminosity ultra-long bursts like GRB 060218/GRB 100316D, with echo-like afterglows implying a modest amount of dust; 2) spiky light curve, somewhat low-luminosity ultra-long bursts like GRB 130925A/GRB 090417B, with echo-like afterglows implying a large 
amount of dust; and 3) spiky-light curve bursts with typical timescale and luminosity, and synchrotron afterglows.

The underlying reason why the afterglow is dominated by dust-scattered prompt emission in some cases, and synchrotron emission from external shocks in others, is unclear. One possibility is that kinetic energy is efficiently converted to radiation during the prompt phase, resulting in a lower kinetic energy during the afterglow phase as discussed by Evans et al. (2014) in the context of GRB 130925A. A second possibility is that the external shocks do not effectively couple energy to postshock electrons and/or magnetic fields. We return to this question at the end of Section 2.4.7.

\subsubsection{Radio afterglow}

An essential feature of the radio afterglow in GRB 060218 is that it shows no evidence for a jet break, but instead decays as a shallow power law in time, with $F_{\nu} \propto t^{-0.85}$ at 22.5 GHz (Soderberg et al. 2006). This behavior runs contrary to analytical models of GRB radio afterglows (e.g., Rhoads 1999; Sari et al. 1999) which predict that, after a relatively flat decay during the Blandford-McKee phase, the on-axis light curve should break steeply to $t^{-p}$ after a critical time $t_{j}$. Here, $p$ is the power law index of accelerated postshock electrons, i.e. $N(E) \propto E^{-p}$, which typically takes on values $2<$ $p<3$. The steepening is due to a combination of two effects that reduce the observed flux: when the jet decelerates to $\Gamma \sim \theta_{0}$, the jet edge comes into view, and also the jet begins to expand laterally. The same general behavior of the radio light curve is also seen in numerical simulations (Zhang \& MacFadyen 2009; van Eerten \& MacFadyen 2013). The steep decay lasts until a time $t_{s}$, which is the timescale for the flow to become quasi-spherical if sideways expansion is fast, i.e. if the increase in radius during sideways expansion is negligible (Livio \& Waxman 2000). While detailed 
simulations have demonstrated that the transition to spherical outflow is much more gradual and that the flow remains collimated and transrelativistic at $t_{s}$ (Zhang \& MacFadyen 2009; van Eerten \& MacFadyen 2012), numerical light curves nonetheless confirm that analytical estimates of the radio flux that assume sphericity and nonrelativistic flow remain approximately valid for on-axis observers at $t>t_{s}$ (van Eerten \& MacFadyen 2012; Wygoda et al. 2011). After $t_{s}$, the light curve gradually flattens as the flow tends toward the Sedov-Taylor solution, eventually becoming fully nonrelativistic on a timescale $t_{N R}$. Therefore, the smooth and relatively flat light curve of GRB 060218 over the period 2-20 days suggests one of two possibilities: either we observed the relativistic phase of an initially wide outflow that took $t_{j} \gtrsim 20$ days to enter the steep decay phase, or we observed the late phase of an outflow that became transrelativistic in $t_{s} \lesssim 2$ days and that may have been beamed originally.

In either scenario, a light curve as shallow as $t^{-0.85}$ is not easily produced in the standard synchrotron afterglow model. One issue is that such a shallow decay suggests that the circumstellar density profile and postshock electron spectrum are both flatter than usual. Throughout the period of radio observations, the characteristic frequency $\nu_{m}$, the synchrotron self-absorption frequency $\nu_{a}$, and the cooling frequency $\nu_{c}$ are related by $\nu_{m}<\nu_{a}<\nu_{c}$ (Soderberg et al. 2006). As the $22.5 \mathrm{GHz}$ band lies between $\nu_{a}$ and $\nu_{c}$, the expected light curve slope in the relativistic case is $t^{3(1-p) / 4}$ for a constant density circumstellar medium, and $t^{(1-3 p) / 4}$ for a wind-like medium (Leventis et al. 2012; Fan et al. 2006). In the non-relativistic limit, the slopes are $t^{3(7-5 p) / 10}$ (constant density) and $t^{(5-7 p) / 6}$ (wind) (Leventis et al. 2012). In order to fit the observed slope $t^{-0.85}$, we require a constant density medium and $p=2.1$ (relativistic) or $p=2.0$ (non-relativistic). However, Panaitescu \& Kumar (2002) found that the afterglows of several typical GRBs were best explained with a constant density model, and a low 
$p$-value was indicated for a number of bursts in their sample. Hence, GRB 060218 does not seem so unusual in this regard.

A second point of tension with the shallow light curve is the observed Lorentz factor. Soderberg et al. (2006) inferred a mildly relativistic bulk Lorentz factor $\Gamma \simeq 2.4$ from an equipartition analysis. However, they based their analysis on the treatment of Kulkarni et al. (1998), which did not include the effects of relativistic expansion. A more accurate calculation that takes relativistic and geometrical effects into account was carried out by Barniol Duran et al. (2013). From Figure 2 in Soderberg et al. (2006), we estimate that, at day 5 , the spectral flux at peak was $F_{p} \sim 0.3 \mathrm{mJy}$ and the peak frequency was $\nu_{p}=\nu_{a} \sim 3 \mathrm{GHz}$. Applying equation (5) in Barniol Duran et al. (2013), we obtain a bulk Lorentz factor $\Gamma \approx 0.8$. On the other hand, using their equation (19) for the equipartition radius $R_{N}$ in the non-relativistic limit, we find $\beta \sim R_{N} / c t \approx 1.3$. These results indicate that the outflow is in the mildly relativistic $(\beta \Gamma \sim 1)$ limit, where neither the Blandford-McKee solution (which applies when $\Gamma \gg 2$ ) nor the Sedov-Taylor solution (which applies when $\beta \ll 1$ ) is strictly valid. As discussed above, one expects a relatively shallow light curve slope in these limits, but during the transrelativistic transitional regime the slope tends to be steeper. In spite of these caveats, we press on and compare the relativistic and non-relativistic limits of the standard synchrotron model.

The possibility of a wide, relativistic outflow was first considered by Soderberg et al. (2006). Their spherical relativistic blast wave model predicts an ejecta kinetic energy $E_{k} \sim 2 \times 10^{48} \mathrm{ergs}$ and a circumburst density $n \sim 100 \mathrm{~cm}^{-3}$, assuming fractions $\epsilon_{e} \sim 0.1$ and $\epsilon_{B} \sim 0.1$ of the postshock energy going into relativistic electrons and magnetic fields, respectively. In order to postpone the jet break, they presumed the initial outflow to be wide, with $\theta_{0} \gtrsim 1.4$ (Soderberg et al. 2006). Yet, as Toma et 
al. (2007) pointed out, given the isotropic equivalent $\gamma$-ray energy $6 \times 10^{49} \mathrm{ergs}$, the parameter set of Soderberg et al. (2006) predicts an unreasonably high $\gamma$-ray efficiency, $\eta_{\gamma} \approx 98 \%$. Fan et al. (2006) refined this analysis and showed that parameters $E_{k} \sim$ $10^{50} \mathrm{ergs}, n \sim 100 \mathrm{~cm}^{-3}, \epsilon_{e} \sim 10^{-2}$, and $\epsilon_{B} \sim 10^{-3}$ also fit the data while keeping the $\gamma$-ray efficiency within reason, but the origin of the spherical (or very wide) relativistic outflow is still unclear. One possibility is that the some fraction of the SN ejecta is accelerated to relativistic speeds. However, Tan et al. (2001) have found that, even for a large SN energy $\sim 10^{52}$ ergs, only a fraction $\sim 10^{-4}$ goes into relativistic ejecta. It therefore seems implausible that $\sim 5 \%$ of the SN energy $2 \times 10^{51}$ ergs could be coupled to relativistic material in GRB 060218. A choked jet in a low-mass envelope, as discussed by Nakar (2015), provides an alternative way to put significant energy into a quasi-spherical, relativistic flow.

Given the difficulties with the relativistic scenario, Toma et al. (2007) considered the possibility that the radio emission comes from the late spherical phase of an originally collimated outflow instead. With the same assumption of $\epsilon_{e}=\epsilon_{B}=0.1$, Toma et al. (2007) infer the same kinetic energy and circumstellar density as Soderberg et al. (2006). The advantage of their view is that it eliminates the efficiency problem, as the isotropic equivalent kinetic energy during the early beamed phase is larger by a factor $2 / \theta_{0}^{2}$.

Barniol Duran et al. (2015) also looked at a mildly relativistic synchrotron model in the context of $\mathrm{SN}$ shock breakout. In this case, the light curve decays more slowly since energy is continuously injected as the outer layers of the SN ejecta catch up to the shocked region. As a result, the radio light curve is better fit by a wind profile than a constant density in the breakout case (Barniol Duran et al. 2015). Their study adopts a fixed $\epsilon_{e}=0.2$, and a fixed energy and Lorentz factor for the fast shell 
dominating breakout emission, $E_{f}=2 \times 10^{50} \mathrm{ergs}$ and $\gamma_{f}=1.3$, which are derived from the relativistic breakout model of Nakar \& Sari (2012). They then vary $\epsilon_{B}$ and the wind density parameter $A_{*}$, concluding that $\epsilon_{B}=1.5 \times 10^{-4}$ and $A_{*}=10$ give the best fit. Due to degeneracy, however, other parameter sets with different energy and $\epsilon_{e}$ may fit the radio light curves as well.

Unfortunately, such degeneracies involving the unknown quantities $\epsilon_{e}$ and $\epsilon_{B}$ are an unavoidable limitation when deriving $E_{k}$ and $n$ in the standard synchrotron model. The available observations give only the specific flux $F_{\nu}$, the self-absorption frequency $\nu_{a}$, and an upper limit on the cooling frequency $\nu_{c}$, which is not sufficient to uniquely determine the four model parameters. In practice, this is typically addressed by fixing two of the parameters to obtain the other two. (For example, Soderberg et al. (2006) choose $\epsilon_{e}$ and $\epsilon_{B}$; Barniol Duran et al. (2015) fix $\epsilon_{e}$ and $E_{k}$.) We take a different approach. In this section and Section 2.4.6, we consider a number of constraints from dynamics, timescales, and direct radio, optical, and X-ray observations, assuming that the emission is from the late phase of an initially collimated jet. We apply these conditions to constrain the available $\left(E_{k}, n, \epsilon_{e}, \epsilon_{B}, \theta_{0}, \gamma\right)$ parameter space. We then consider whether any reasonable parameter set is consistent with a jet that could produce the observed thermal X-rays through dissipation at early times, as described in Section 2.4.1.

We begin with the constraints inferred directly from radio observations. We have $\nu_{a} \sim 4 \times 10^{9} \mathrm{~Hz}$ at 5 days, $F_{\nu}(22.5 \mathrm{GHz}) \sim 0.25 \mathrm{mJy}$ at 3 days, and $\nu_{c} \lesssim 5 \times$ $10^{15} \mathrm{~Hz}$ so that the synchrotron flux remains below the observed X-ray afterglow flux throughout observations (Soderberg et al. 2006). Lower limits on $E_{k}$ and $n$ can be deduced by assuming $\epsilon_{e}<1 / 3$ and $\epsilon_{B}<1 / 3$. For a relativistic blast wave with $p=2.1$, we have $\epsilon_{B,-2}^{0.34} \epsilon_{e,-1}^{0.36} C_{p}^{0.36} E_{k, 51}^{0.34} n^{0.33} \sim 0.44, \epsilon_{B,-2}^{0.78} \epsilon_{e,-1}^{1.1} C_{p}^{1.1} E_{k, 51}^{1.28} n^{0.5} \sim 0.0032$, and 
$\epsilon_{B,-2}^{-1.5} E_{k, 51}^{-0.5} n^{-1} \lesssim 0.43$ (Fan et al. 2006), where $C_{p}=13(p-2) / 3(p-1) \approx 0.39$. In this case, we find $E_{k} \gtrsim 7 \times 10^{47}$ ergs and $n \gtrsim 3 \mathrm{~cm}^{-3}$. Similarly, in the non-relativistic limit Toma et al. (2007) derived $\epsilon_{B,-2}^{1 / 3} \epsilon_{e,-1}^{1 / 3} E_{k, 51}^{1 / 3} n^{1 / 3} \sim 1, \epsilon_{B,-2}^{3 / 4} \epsilon_{e,-1} E_{k, 51}^{1.3} n^{0.45} \sim 0.003$, and $\epsilon_{B,-2}^{3 / 2} E_{k, 51}^{3 / 5} n^{0.9} \gtrsim 0.4$. This leads to the constraints $E_{k} \gtrsim 1 \times 10^{47}$ ergs and $n \gtrsim 60 \mathrm{~cm}^{-3}$.

The minimum synchrotron energy $E_{\min }$ provides a further constraint on burst energetics. In general, calculating $E_{\min }$ requires integrating the specific synchrotron luminosity $L_{\nu}$ over a range of frequencies $\nu_{\min }-\nu_{\max }$. However, when $p \simeq 2.5$, the dependence of $E_{\min }$ on $\nu_{\min }, \nu_{\max }$, and $p$ is weak (Longair 2011). In that case, if $L_{\nu}$ is measured at frequency $\nu$, one can obtain a rough estimate of $E_{\min }$ by setting $\nu=\nu_{\min }$ : with quantities given in cgs units, $E_{\text {min }} \simeq 8.0 \times 10^{13}(1+\eta)^{4 / 7} V^{3 / 7} \nu^{2 / 7} L_{\nu}^{4 / 7} \mathrm{ergs}$ (Longair 2011), where $\eta$ is the ratio of proton energy to electron energy, which is not known. Soderberg et al. (2006) estimated that the size of the radio-emitting region is $R=$ $3 \times 10^{16} \mathrm{~cm}$ at $t=5$ days, so the emitting volume at that time can be approximated by $V \sim \frac{4}{3} \pi R^{3} \sim 1.1 \times 10^{50} \mathrm{~cm}^{3}$. At the same time, the flux density at $\nu=4.86 \mathrm{GHz}$ was $S_{\nu}=300 \mu \mathrm{Jy}$, implying a specific luminosity $L_{\nu}=7.5 \times 10^{27} \mathrm{erg} \mathrm{s}^{-1} \mathrm{~Hz}^{-1}$ given the distance $D=145 \mathrm{Mpc}$. With these parameters, we find $E_{\min } \sim 1.2 \times 10^{47}(1+$ $\eta)^{4 / 7}$ ergs. Compared to the above estimate, this puts a stricter lower limit on the energy when $\eta$ is large.

A further condition comes from timescale considerations, since the steep $t^{-p}$ part of the light curve should fall outside of the observational period. For an on-axis observer, a numerically calibrated expression for the jet break time in the observer frame is $t_{j}=3.5 E_{i s o, 53}^{1 / 3} n^{-1 / 3}\left(\theta_{0} / 0.2\right)^{8 / 3}$ days (van Eerten et al. 2010). In the relativistic case, we need $t_{j} \gtrsim 20$ days, so $E_{k, 51}^{1 / 3} n^{-1 / 3}\left(\theta_{0} / 0.2\right)^{2} \gtrsim 5.7$. On the other hand, the time $t_{s}$ that roughly marks the end of the steep light curve phase is $t_{s} \simeq 365 E_{\text {iso }, 53}^{1 / 3} n^{-1 / 3}\left(\theta_{0} / 0.2\right)^{2 / 3}$ days (Livio \& Waxman 2000). Since $t_{s} \lesssim 2$ is needed 
for the non-relativistic model, we have $E_{k, 51}^{1 / 3} n^{-1 / 3} \lesssim 0.0055$. Note that $t_{j} \sim\left(\theta_{0}^{2} / 4\right) t_{s}$.

For typical burst energies and CSM densities, the relativistic scenario requires a very wide opening angle to make $t_{j}$ sufficiently large. For example, the parameters of Soderberg et al. (2006) require $\theta_{0} \gtrsim 80^{\circ}$. An equally large $\theta_{0}$ is inferred for GRB 100316D. In that object, the radio afterglow has a similar slow temporal decay, but the timescale of the $F_{\nu}$ peak at $8.5 \mathrm{GHz}$ was $\sim 10$ times longer, occurring at 30 days (Margutti et al. 2013) as compared to 3 days in GRB 060218 (Soderberg et al. 2006), and the radio luminosity is about 10 times higher at 20 days (Margutti et al. 2013). Assuming the same microphysics, this implies about the same burst energy, but a circumstellar density that is higher by a factor of 100-1000 (Margutti et al. 2013), even for a quasi-spherical outflow. It seems unusual that the progenitors of these similar bursts have such different circumstellar environments. In addition, the higher density leads to a smaller $t_{j}$ than in GRB 060218, while radio observations show a flat light curve over the period 20-70 days (Margutti et al. 2013) implying $t_{j} \gtrsim 70$ days, larger than GRB 060218. This problem is alleviated by considering a wind-like medium as in Margutti et al. (2013), but in that case the expected light curve is $\propto t^{-3 / 2}$ for $p=2$, which seems too steep to fit observations unless one adopts $p<2$.

One can consider the non-relativistic case instead, but due to the weak dependence on $E_{k}$ and $n$, the condition on $t_{s}$ is also hard to satisfy unless the burst energy is extremely low or the CSM is extremely dense. In addition, because the flow is still highly aspherical at $t \sim t_{s}$, the model light curve slope will be too steep if $t \gtrsim t_{s}$ only holds marginally, even if the flux is approximately correct. The slope does not settle to the limiting Sedov-Taylor value until the outflow sphericizes, which according to numerical simulations does not occur until $\sim 5 t_{N R} \simeq 4700 E_{k, 51}^{1 / 3} n^{-1 / 3}\left(\theta_{0} / 0.2\right)^{-2 / 3}$ days $\gg t_{s}$ 
(Zhang \& MacFadyen 2009; van Eerten \& MacFadyen 2012).

However, so far we have assumed that the CSM near the progenitor star is the same as the CSM at $\sim$ a few $10^{16} \mathrm{~cm}$ where the radio is emitted. It is possible that the circumstellar environment is more complicated, and in particular that the CSM density is higher closer to the progenitor star. In fact, there is some evidence that this is the case. The X-ray absorption column, $N_{H} \approx 6 \times 10^{21} \mathrm{~cm}^{-2}$, measured during the prompt phase is higher than one would expect for a constant density medium with $n \sim 100 \mathrm{~cm}^{-3}$, even if that medium extended to $\sim 10 \mathrm{pc}$ scales. Thus, we speculate that the shell emitting the radio could have undergone additional deceleration by sweeping up the material responsible for X-ray absorption at some time $t<2$ days. While the absorbing column through the expanding outflow is expected to change with time, the measured $N_{H}$ is constant during the prompt phase. Therefore, the bulk of the absorbing material would have to lie outside the jet throughout the first $10^{4} \mathrm{~s}$. Note that $N_{H}$ also stays constant throughout the X-ray afterglow from $10^{4}-10^{6} \mathrm{~s}$, but this does not provide any additional constraints on our model, since the afterglow in our picture is a light echo and thus inherits the absorption of the prompt component (see Section 2.4.4). Hence most of the absorbing mass must be confined to the radial range $R\left(10^{4} \mathrm{~s}\right)<R_{a b s}<R(2$ days $)$. Taking $R \sim 2 \Gamma^{2} c t$, where $\Gamma$ is the Lorentz factor of the forward shock, we find $0.002 \mathrm{pc} \lesssim R_{a b s}(\Gamma / 3)^{-2} \lesssim 0.03 \mathrm{pc}$. The total mass of absorbing material, assuming it is distributed isotropically, is $M_{a b s} \sim 4 \pi R_{a b s}^{2} N_{H} m_{p}$, so $0.002 M_{\odot} \lesssim M_{a b s}(\Gamma / 3)^{-4} \lesssim 0.5 M_{\odot}$. Note that less absorbing mass is necessary if it is located closer to the star, or if it is distributed preferentially along the poles. The jet will sweep a mass $M_{s w} \sim\left(\theta_{0}^{2} / 2\right) M_{a b s}$, which is sufficient to decelerate it to nonrelativistic speeds if $M_{s w} \gg M_{j}$. From equation $(2.4), M_{j} \sim 10^{-4}\left(\theta_{0}^{2} / 2\right)(\gamma / 3)^{3} M_{\odot}$ for 
the parameters of GRB 060218, thus we find

$$
7 \Gamma(\Gamma / \gamma)^{3} \lesssim M_{s w} / M_{j} \lesssim 2000 \Gamma(\Gamma / \gamma)^{3} .
$$

$M_{s w} / M_{j}>1$ is possible to satisfy for $\Gamma \gtrsim 0.1 \gamma^{4 / 3}$, and is always satisfied for $\Gamma \gtrsim 0.6 \gamma^{4 / 3}$. Therefore, for the mildly relativistic case we consider, it seems plausible that the mass responsible for the X-ray absorption could also be responsible for decelerating the jet.

The conditions $\epsilon_{e}<1 / 3, \epsilon_{B}<1 / 3, E_{k}>E_{\min }, \nu_{c}<5 \times 10^{15} \mathrm{~Hz}$, and the constraint on $t_{j}$ (or $t_{s}$ ) can be conveniently expressed in the $\epsilon_{e}-\epsilon_{B}$ plane. The result is shown in Figure 2.4. (We do not plot the line $E_{k}=E_{\min }$ as it is largely irrelevant as long as $\eta \sim 1$.) The first two panels show the standard, constant CSM density case, for a relativistic flow with $p=2.1$ and a non-relativistic flow with $p=2.0$, respectively. In the relativistic case, if the jet is very wide $\left(\theta_{0} \simeq 1.4\right)$, the $t_{j}$-condition can be marginally satisfied ( $t_{j} \gtrsim 10$ days) for $\epsilon_{B} \gtrsim \epsilon_{e}$ as depicted in the top panel. However, because $t_{j}$ is sensitive to $\theta_{0}$, the available parameter space rapidly shrinks when $\theta_{0}$ is reduced: the green region disappears from the plot when $\theta_{0} \lesssim 1.0$, and the yellow region when $\theta_{0} \lesssim 0.7$. Thus, the relativistic scenario disfavors a tightly collimated outflow for any sensible combination of $\epsilon_{e}$ and $\epsilon_{B}$. In the non-relativistic case, shown in the middle panel, we see that the constraints $t_{s}<2$ days and $\nu_{c}<5 \times 10^{15} \mathrm{~Hz}$ can not be jointly satisfied for any choice of parameters. At best, the $t_{s}$-condition can be met marginally ( $t_{s} \lesssim 4$ days) if $10^{-3} \lesssim \epsilon_{B} / \epsilon_{e} \lesssim 10^{-1}$. (Here, we also show the condition $t(\beta=1)<2$ days, which was used by Toma et al. (2007). However, as discussed above and in Wygoda et al. (2011), the radio flux still deviates considerably from the Sedov-Taylor prediction at this time because the outflow is semirelativistic.) The situation changes if some additional mass is swept up by the jet before the 
first radio observation, in which case the condition $E_{k}<M_{s w} c^{2}$ replaces the upper limit on $t_{s}$. This scenario is shown in the bottom panel, assuming $M_{s w}=10^{-5} M_{\odot}$ (corresponding to an isotropic mass $10^{-3} M_{\odot}$ for $\theta_{0}=0.2$ ). Compared to the standard cases, this case accommodates a larger set of possible parameters. The effect of increasing (decreasing) $M_{s w}$ is to increase (decrease) the size of the green region by moving the critical line $E_{k}=M_{s w} c^{2}$ towards the lower left (upper right).

\subsubsection{Jet propagation}

We now examine the evolution of the jet as it drills the star and breaks out into the surrounding medium. For our picture so far to be plausible, several conditions must be met. First, the initial kinetic energy of the outflow $E_{i s o}$ must exceed the prompt isotropic radiated energy $E_{\gamma, \text { iso }}=6 \times 10^{49} \mathrm{ergs}$, i.e. the radiative efficiency $\eta_{\gamma}=$ $E_{\gamma, i s o} / E_{i s o}<1$. Using equation 2.5, this implies $\gamma \gtrsim 2$. Note that $E_{i s o}=E_{\gamma_{i} s o}+E_{k, i s o}$, where $E_{k, i s o}=\left(2 / \theta_{0}^{2}\right) E_{k}$. Second, the total breakout time from the stellar core and extended envelope, $t_{b}=t_{b, *}+t_{b, e x t}$, should be shorter than the duration of prompt X-rays $t_{L}$. Third, the interaction with the extended envelope should be dominated by the supernova, and not by the jet or cocoon. In other words, the jet/cocoon system should not sweep up or destroy the envelope before the supernova has a chance to interact with it. Finally, we expect that the energy in relativistic ejecta will be less than the SN energy: $E_{j}<E_{S N}$.

In what follows, we scale the collimation-corrected jet luminosity $L_{j}$ to $10^{46} \mathrm{erg} \mathrm{s}^{-1}$, corresponding to a jet energy $E_{j} \sim L_{j} t_{L} \sim 3 \times 10^{49}$ ergs. We assume a constant jet luminosity for simplicity. (A time-varying luminosity does not affect our general conclusions, as long as the average value of $L_{j}$ remains the same.)

In order for such a low-luminosity jet to penetrate the progenitor star, Toma 

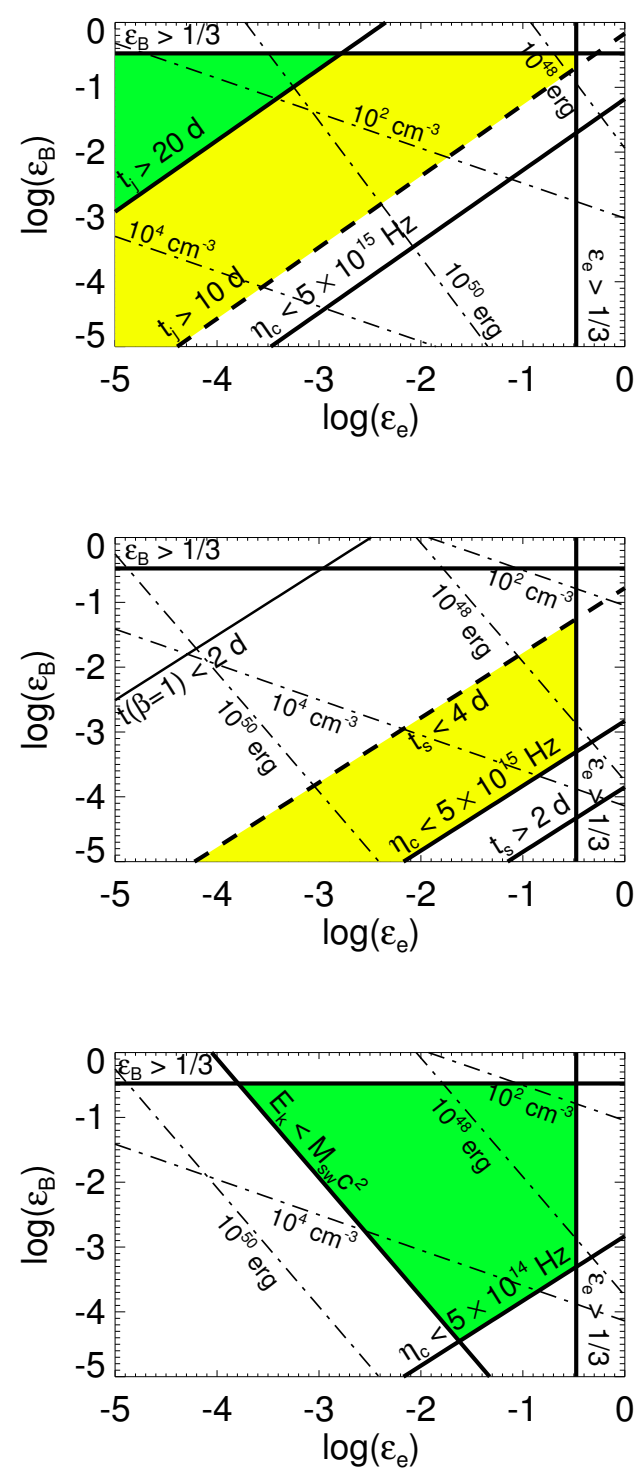

Fig. 2.4. - Constraints described in Section 2.4.5, depicted in the $\epsilon_{e}-\epsilon_{B}$ plane. In each plot, thin dash-dotted lines of constant $E_{k}\left(10^{48} \mathrm{ergs}\right.$ and $\left.10^{50} \mathrm{ergs}\right)$ and $n\left(10^{2} \mathrm{~cm}^{-3}\right.$ and $10^{4} \mathrm{~cm}^{-3}$ ) are drawn. The conditions $\epsilon_{B}=1 / 3, \epsilon_{e}=1 / 3$, and $\nu_{c}=5 \times 10^{15} \mathrm{~Hz}$ are shown as heavy solid lines, as labeled in the diagram. Regions where all conditions are met are shaded in green, while yellow regions indicate that the conditions are met if the timescale constraints are relaxed by a factor of 2. Top: The relativistic case with $p=2.1$. The condition $t_{j}=20$ days is shown, assuming a wide jet $\left(\theta_{0} \simeq 1.4\right)$. Middle: The non-relativistic case with $p=2.0$, assuming the same density for all $r>R_{\text {ext }}$, with the conditions $t_{s}=2$ days, $t_{s}=4$ days, and $t(\beta \simeq 1)=2$ days. Note that $t_{s} \lesssim 2$ days and $\nu_{c}<5 \times 10^{15} \mathrm{~Hz}$ cannot be jointly satisfied. Bottom: The nonrelativistic case, assuming some additional mass $M_{s w}=10^{-5} M_{\odot}$ is swept up prior to 2 days. See the text for discussion. 
et al. (2007) found that it must be hot and have a narrow opening angle $\theta_{j} \lesssim 0.03$, conditions that are satisfied by a collimated jet. The general theory of jet propagation in the collimated and uncollimated regimes was put forth by Bromberg et al. (2011). Their model is applicable when the jet is injected with a Lorentz factor $\gamma_{i n j}$ and opening angle $\theta_{i n j}$ that satisfy $\gamma_{i n j} \gtrsim \theta_{i n j}^{-1}$. They showed that the jet is collimated if $L_{j}<\pi r_{h}^{2} \rho_{a} c^{3} \theta_{i n j}^{2 / 3}$, where $r_{h}$ is the radius of jet's head and $\rho_{a}$ is the density of the ambient medium. For a typical WR star with mass $M_{*}=10 M_{\odot}$ and radius $R_{*}=10^{11} \mathrm{~cm}$, the jet is collimated for $L_{j} \lesssim 10^{52} \mathrm{erg} \mathrm{s}^{-1}$ (Bromberg et al. 2011), so we are well within this regime. While propagating in the star, collimation by the uniform-pressure cocoon keeps the jet cross section approximately constant, and the Lorentz factor below the jet head is $\gamma_{j} \sim \theta_{i n j}^{-1}$, independent of the injection Lorentz factor $\gamma_{i n j}$ (Bromberg et al. 2011). Later, once the jet breaks out into a low-density medium, it becomes uncollimated and its opening angle and Lorentz factor tend towards $\sim \theta_{i n j}$ and $\sim \gamma_{i n j}$, respectively (Bromberg et al. 2011). Therefore, the values $\theta_{0}$ and $\gamma$, which describe the jet post-breakout, provide an estimate of the injection conditions at much smaller radii, i.e. $\theta_{i n j} \sim \theta_{0}$ and $\gamma_{i n j} \sim \gamma$. As a result,

$$
\gamma \gtrsim \theta_{0}^{-1}
$$

will hold after adiabatic expansion.

In the strongly collimated limit the jet head moves non-relativistically with speed $\beta_{h} \simeq L_{j}^{1 / 5} \rho_{a}^{-1 / 5} t^{-2 / 5} \theta_{i n j}^{-4 / 5} c^{-1}$ (Bromberg et al. 2011). Let the stellar density profile be $\rho_{a}=\rho_{0}\left(r / R_{*}\right)^{-\delta_{*}}$, with $\rho_{0}=\frac{\left(3-\delta_{*}\right) M_{*}}{4 \pi R_{*}^{3}}$. Typically, $1.5 \lesssim \delta_{*} \lesssim 3$ for WR stars (e.g., Matzner \& McKee 1999). Equation (B-2) in Bromberg et al. (2011) gives the radius of the jet head as a function of time for the case of a non-relativistic head; 
substituting $r=R_{*}$ and $\rho_{a}=\rho_{0}$ into that expression, we find the breakout time

$$
t_{b, *} \simeq 90 C\left(\delta_{*}\right) L_{j, 46}^{-1 / 3} M_{c, 1}^{1 / 3} R_{c, 11}^{2 / 3}\left(\frac{\theta_{0}}{0.2}\right)^{4 / 3} \mathrm{~s} .
$$

The order-unity constant $C\left(\delta_{*}\right)=\left[\frac{3}{7} \frac{5-\delta_{*}}{3-\delta_{*}}\right]^{-2 / 3}$ scales the result to $\delta_{*}=1.5$, as this gives the most conservative estimate of the breakout time for the typical range of $\delta_{*}$.

Breakout from the low-mass envelope proceeds similarly to breakout from the stellar core, the main differences being that the jet head is faster and harder to collimate due to the lower ambient density. For an envelope density profile $\rho_{\text {ext }} \propto$ $r^{-\delta_{e x t}}, \rho_{e x t}\left(R_{e x t}\right)=\frac{\left(3-\delta_{e x t}\right) M_{e x t}}{4 \pi R_{e x t}^{3}}$. While $\delta_{e x t}$ is not known in general, requiring that the density decreases outwards and that most of the envelope mass is at large radii restricts its value to $0 \leq \delta_{\text {ext }} \leq 3$. The collimation condition at the edge of the envelope can be rewritten as

$$
L_{j, 46} \lesssim 50\left(3-\delta_{e x t}\right) M_{e x t,-3} R_{e x t, 13}^{-1}\left(\frac{\theta_{0}}{0.2}\right)^{2 / 3} \equiv L_{c o l l, 46}
$$

For our parameters, we find that the jet remains collimated throughout the envelope, though we note that the high-luminosity jet of a typical GRB would be uncollimated in the same envelope (as discussed by Nakar 2015). The parameter $\tilde{L}=\left(L_{j} / \rho_{\text {ext }} t^{2} \theta_{0}^{4} c^{5}\right)^{2 / 5}$ then determines if the jet head is relativistic $(\tilde{L}>1)$ or Newtonian $(\tilde{L}<1)$ (Bromberg et al. 2011). The condition for a non-relativistic head at breakout is

$$
L_{j, 46} \lesssim 7 \times 10^{-2}\left(3-\delta_{e x t}\right) M_{e x t,-3} R_{e x t, 13}^{-1}\left(\frac{\theta_{0}}{0.2}\right)^{4} .
$$

For the range of parameters we consider, the jet is usually relativistic at the time of breakout; however, for a low luminosity or a somewhat wide opening angle, it is 
possible that the jet head breaks out non-relativistically.

Since we are interested in a lower bound on the jet luminosity, we compute the breakout time in the non-relativistic limit. In this case, we can reuse equation (2.8) with $R_{*}$ and $M_{*}$ replaced by $R_{e x t}$ and $M_{e x t}$ to calculate the breakout time from the envelope. We have

$$
t_{b, e x t} \simeq 120 C\left(\delta_{e x t}\right) L_{j, 46}^{-1 / 3} M_{e x t,-3}^{1 / 3} R_{e x t, 13}^{2 / 3}\left(\frac{\theta_{0}}{0.2}\right)^{4 / 3} \mathrm{~s}
$$

where in this case we have scaled to $\delta_{e x t}=0$ via $C\left(\delta_{e x t}\right)=\left[\frac{3}{5} \frac{5-\delta_{e x t}}{3-\delta_{e x t}}\right]^{2 / 3}$, which maximizes $t_{b, e x t}$. Combining equations (2.8) and (2.11) with the parameters $M_{c} \approx$ $2 M_{\odot}, M_{e x t} \approx 4 \times 10^{-3} M_{\odot}$, and $R_{e x t} \approx 9 \times 10^{12} \mathrm{~cm}$ inferred in Section 2.4.3, and an assumed core radius $R_{c} \sim 10^{11} \mathrm{~cm}$, we find the total breakout time

$$
t_{b} \simeq 230 L_{j, 46}^{-1 / 3}\left(\frac{\theta_{0}}{0.2}\right)^{4 / 3} \mathrm{~s}
$$

The condition $t_{b}<t_{L}$ is satisfied as long as $L_{j, 46} \gtrsim 5 \times 10^{-4}\left(\theta_{0} / 0.2\right)^{4}$, which generally holds in our model so long as the jet is reasonably beamed.

To ensure that the interaction with the envelope is dominated by the supernova ejecta, the supernova energy should exceed the energy of the jet-blown cocoon, so that the former overtakes the latter. The energy deposited into the cocoon up to breakout is $E_{c} \sim L_{j}\left(t_{b}-R_{b} / c\right)$ (Lazzati et al. 2015), where $R_{b}$ is the breakout radius. There are two dynamically distinct cocoons that can potentially disturb the stellar envelope. First, while the jet is within the stellar core, material entering the jet head escapes sideways to form a cocoon of shocked stellar matter. When the jet breaks out of the stellar core and enters the surrounding envelope, this "stellar cocoon" also breaks out and begins to sweep the envelope as it expands outwards. Then, 
as the jet continues to propagate through the envelope, it blows a second cocoon containing shocked envelope material. This "envelope cocoon" expands laterally as the jet propagates, and then breaks out into the circumstellar medium once the jet reaches the envelope's edge. Here, we show that these cocoons have a negligible effect on the envelope dynamics compared to the supernova, because the stellar cocoon is too slow and the envelope cocoon is too narrow.

Consider first the stellar cocoon. While traversing the star, the jet head is nonrelativistic, so $t_{b} \gg R_{b} / c$ and essentially all of the energy goes into the cocoon, i.e. $E_{c, *} \sim L_{j} t_{b, *}$. From equation (2.8), we have

$$
E_{c, *} \sim 3 \times 10^{48} L_{j, 46}^{2 / 3} M_{c, 1}^{1 / 3} R_{c, 11}^{2 / 3}\left(\frac{\theta_{0}}{0.2}\right)^{4 / 3} \text { ergs. }
$$

Here and for the rest of this section, we ignore order-unity factors that depend on $\delta_{*}$ or $\delta_{\text {ext }}$. When $\beta_{h}<1$, the cocoon expands sideways with speed $\beta_{c} \approx \beta_{h} \theta_{0}$, resulting in a cocoon opening angle $\theta_{c} \sim \beta_{c} / \beta_{h} \sim \theta_{0}$ (Bromberg et al. 2011). The mass entrained in the cocoon at the time of breakout is therefore

$$
M_{c, *} \sim \frac{\theta_{0}^{2}}{2} M_{c} \sim 0.2 M_{c, 1}\left(\frac{\theta_{0}}{0.2}\right)^{2} M_{\odot}
$$

After breakout the cocoon material expands with typical speed $v_{c, *} \sim\left(2 E_{c, *} / M_{c, *}\right)^{1 / 2}$, so we have

$$
v_{c, *} \sim 10^{8} L_{j, 46}^{1 / 3} M_{c, 1}^{-1 / 3} R_{c, 11}^{1 / 3}\left(\frac{\theta_{0}}{0.2}\right)^{-1 / 3} \mathrm{~cm} \mathrm{~s}^{-1}
$$

This is generally much slower than $v_{\text {ext }} \approx 3 \times 10^{9} \mathrm{~cm} \mathrm{~s}^{-1}$ in our model, with $v_{c, *}<v_{\text {ext }}$ for $L_{j, 46} \lesssim 3 \times 10^{4} M_{c, 1} R_{c, 11}^{-1}\left(\theta_{0} / 0.2\right)$. Therefore, the fast supernova ejecta rapidly overtake the stellar cocoon.

As discussed above, the jet stays collimated in the envelope, but the jet head 
may become relativistic. In this limit, the lateral speed of the cocoon is $\beta_{c} \sim \tilde{L}^{1 / 2} \theta_{0}$ (Bromberg et al. 2011), and since $\beta_{h} \approx 1, \theta_{c} \sim \beta_{c}$. For a collimated jet, $\tilde{L} \lesssim \theta_{0}^{-4 / 3}$ (Bromberg et al. 2011), so we have $\theta_{c} \lesssim \theta^{1 / 3}<1$. As the pressure of the envelope cocoon rapidly drops after it breaks out from the envelope's edge and expands freely into the low-density circumstellar medium, little sideways expansion through the envelope is expected after breakout. Thus, as long as $\theta_{0}$ is small, the passage of the jet and envelope cocoon leaves the envelope relatively intact, and the SN-envelope interaction is quasi-spherical. Note that it is not strictly necessary for the jet to be collimated by the envelope in our model. In principle the jet may be uncollimated, with $\tilde{L}$ somewhat larger than $\theta_{0}^{-4 / 3}$, as long as $\theta_{c}$ remains small, but in practice this regime is not attained in GRB 060218.

Ideally, the jet head should break out of the stellar core before the SN shock. This guarantees that the jet will reach the edge of the envelope before the SN, so the jet will be seen first. Comparing the SN breakout time $t_{S N} \sim R_{*}\left(2 E_{S N} / M_{S N}\right)^{-1 / 2}$ to the breakout time in equation (2.8), one finds

$$
t_{b, *} / t_{S N} \sim 0.6 L_{j, 46}^{-1 / 3} R_{c, 11}^{-1 / 3}\left(\frac{M_{c}}{2 M_{\odot}}\right)^{-1 / 6}\left(\frac{E_{S N}}{2 \times 10^{51} \operatorname{erg~s}^{-1}}\right)^{1 / 2}\left(\theta_{0} / 0.2\right)^{4 / 3}
$$

This condition is satisfied for $L_{j, 46} \gtrsim 0.2\left(\frac{M_{c}}{2 M_{\odot}}\right)^{-1 / 2}\left(\frac{E_{S N}}{2 \times 10^{51} \mathrm{erg} \mathrm{s}^{-1}}\right)^{3 / 2}\left(\theta_{0} / 0.2\right)^{4}$, which is only sometimes met for the parameters considered here. However, even if the SN shock reaches the edge of the star first, the jet breaks out soon after. This is because, after the SN crosses the core, the core density drops as $\rho_{c} \propto\left(v_{S N} t\right)^{-3}$, and as $\beta_{h}$ depends inversely on $\rho_{c}$, the jet soon accelerates to $c \beta_{h}>v_{S N}$. Thus, it may be possible for this constraint to be violated, and we do not rule out models for which $v_{S N}>c \beta_{h}$ initially.

We can get a grasp on the allowed region of $\gamma-\theta_{0}$ parameter space by using equa- 
tions (2.3) and (2.5) to convert the conditions $\eta_{\gamma}<1, E_{j}<E_{S N}, E_{j} \gtrsim E_{\min }, \gamma \gtrsim \theta_{0}^{-1}$, $t_{b} \lesssim t_{L}, v_{c, *}<v_{e x t}$, and $c \beta_{h}<v_{S N}$ to relations between $\gamma$ and $\theta_{0}$. We show the result in Figure 2.5. We see that the available parameter space is bound chiefly by $E_{j}<E_{S N}$ from above, $\gamma \gtrsim \theta_{0}^{-1}$ from the left, $\theta_{0} \lesssim \pi / 2$ from the right, and $\eta_{\gamma} \lesssim 1$ from below. The other important conditions are always satisfied when these four constraints are met. Note that the conditions related to the jet and cocoon do not necessarily apply when $\theta_{0}$ is large, because in that limit the explosion is quasi-spherical instead of jet-like, but the conditions on the overall burst energetics are still relevant. The possible values of $E_{j}, \gamma$, and $\theta_{0}$ lie in the range $7 \lesssim E_{j, 48} \lesssim 2 \times 10^{3}, 2 \lesssim \gamma \lesssim 25$, and $0.04 \lesssim \theta_{0} \lesssim \pi / 2$

Three general classes of solution can satisfy all of the necessary conditions:

- Low kinetic energy, narrow beam, low Lorentz factor: For low jet energies, e.g. $E_{j} \simeq 7 \times 10^{48} \mathrm{erg} \mathrm{s}^{-1}$, the jet is confined to a narrow range around $\theta_{0} \simeq 0.5$ and $\gamma \simeq 2.1$. The isotropic jet energy in this case is $E_{i s o} \simeq$ $6 \times 10^{49} \mathrm{erg} \mathrm{s}^{-1}$, and the radiative efficiency is $\eta_{\gamma} \simeq 0.5$, implying a mildly hot jet. The kinetic energy during the afterglow phase is $E_{k} \simeq 3 \times 10^{48}$, which gives $-2.5 \lesssim \log \epsilon_{e} \lesssim-0.8,-3.6 \lesssim \log \epsilon_{B} \lesssim-0.5$, and $2.5 \lesssim \log n \lesssim 3.9$ using the bottom panel of Fig. 2.4. This solution is similar to that of Toma et al. (2007), who also inferred a mildly hot jet.

- High kinetic energy, narrow beam, high Lorentz factor: For higher kinetic energies, the model is less restrictive: for example, $E_{j}=2 \times 10^{50} \mathrm{ergs}$ gives $0.1 \lesssim \theta_{0} \lesssim 1.5$, allowing for either narrow or wide jets. In the narrow jet case of $\theta_{0} \simeq 0.1$, we have $E_{i s o} \simeq 4 \times 10^{52} \mathrm{erg} \mathrm{s}^{-1}$ and $\gamma \simeq 10$. The radiative efficiency in this case is low, $\eta_{\gamma} \simeq 2 \times 10^{-3}$, and therefore $E_{k} \approx E_{j}$. In order to accommodate the higher energy, this model requires lower-than-standard values 


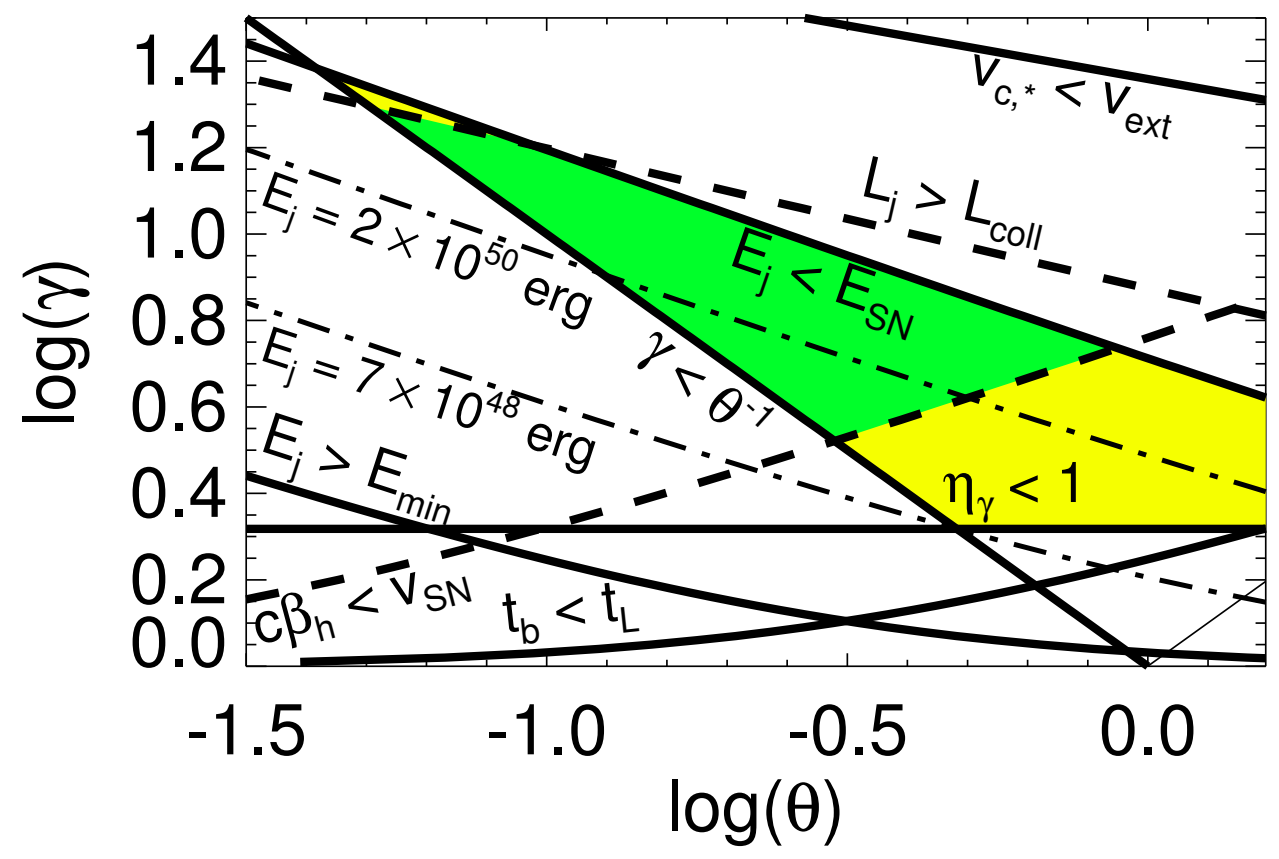

Fig. 2.5.- Constraints described in Section 2.4.6, depicted in the $\theta-\gamma$ plane. The conditions $E_{j}>E_{\text {min }}, E_{j}>E_{S N}, \eta_{\gamma}<1, \gamma \gtrsim \theta^{-1}, t_{b}<t_{L}$, and $v_{c, *}<v_{e x t}$ are drawn as heavy solid lines. Two other constraints that may be marginally violated $\left(L_{j}<L_{\text {coll }}\right.$ and $\left.c \beta_{h}>v_{S N}\right)$ are shown as dashed lines. The regions of parameter space that satisfy all the constraints, and only the strict constraints, are painted green and yellow, respectively. Curves of constant jet energy are shown as thin, dash-dotted lines. See the text for discussion.

for $\epsilon_{e}$ and/or $\epsilon_{B}:-5.3 \lesssim \log \epsilon_{e} \lesssim-2.6$ and $-5.4 \lesssim \log \epsilon_{B} \lesssim-0.5$. For this reason, this scenario has not been considered previously. The CSM density in this case is $3.5 \lesssim \log n \lesssim 5.7$.

- High kinetic energy, wide beam, high Lorentz factor: A high jet energy directed into a wide $\left(\theta_{0} \rightarrow \pi / 2\right)$ outflow is also allowed. In this case $E_{j} \approx E_{\text {iso }}$ . For $E_{j} \simeq 2 \times 10^{50} \mathrm{ergs}$, we find $\gamma \simeq 2.6$ and $\eta_{\gamma} \simeq 0.3$. Using the top panel of Fig. 2.4, we find $-3.9 \lesssim \log \epsilon_{e} \lesssim-2.7,-3.1 \lesssim \log \epsilon_{B} \lesssim-0.5$, and $1.7 \lesssim \log n \lesssim 3.0$ This model is similar to the model proposed by Fan et al. 
(2006), and also consistent with the picture in Nakar (2015), since in that case there is reason to expect a quasi-spherical explosion.

Our picture favors models with a narrow jet, because a wide jet would considerably disrupt the circumstellar envelope, which is problematic for our optical model discussed in Section 2.4.3. However, we do not have a strong reason to prefer a high jet energy versus a low energy one; each option offers some advantage. The low energy case uses typical values for $\epsilon_{e}$ and $\epsilon_{B}$, and more readily transitions to a non-relativistic outflow without the need for an extreme CSM density. On the other hand, the high energy case is compatible with a wider variety of jets with various $\theta_{0}$ and $\gamma$, and requires a less efficient dissipation mechanism since $\eta_{\gamma} \ll 1$.

\subsubsection{Prompt nonthermal emission}

Because GRB 060218 adheres to several well-known GRB correlations, it is worth considering whether this object has the same emission mechanism as standard GRBs. In this section, we attempt to glean as much as possible about the emission mechanism in GRB 060218, assuming that the source of the nonthermal X-rays is a mildly relativistic jet. To do so, we first lay out a simple empirical description for the prompt emission that preserves the essential features of the observed spectrum and light curve. We then consider a number of jet scenarios for the prompt emission.

\section{A simple empirical description of GRB 060218}

The nonthermal emission observed in the XRT and BAT bands appear to have the same origin (Liang et al. 2006), and throughout the prompt phase, the joint BATXRT spectrum is best fit by a Band function (Toma et al. 2007). Here, we aim to find the least complex model that fulfills these criteria while also fitting the light 
curves at various frequencies. Consider a simple Band function model for the prompt nonthermal spectrum, where the photon spectral shape is given by (Band et al. 1993)

$$
\begin{array}{ll}
F(E)=F_{0}\left(E / E_{c}\right)^{\beta_{1}} e^{-E / E_{0}}, & E \leq E_{c} \\
F(E)=F_{0}\left(E / E_{c}\right)^{\beta_{2}} e^{\beta_{2}-\beta_{1}}, & E>E_{c}
\end{array}
$$

The quantities $E_{0}$ and $E_{c}$ are related to the peak energy by $E_{0}=E_{p} /\left(1+\beta_{1}\right)$ and $E_{c}=\left(\beta_{1}-\beta_{2}\right) E_{0}$. Let the spectral indices be constant, and suppose the parameters $E_{p}$ and $F_{0}$ vary as power laws in time, i.e.

$$
E_{p}=E_{n} t^{\alpha_{p}}
$$

and

$$
F_{0}=F_{n} t^{\alpha_{0}}
$$

where $E_{n}$ and $F_{n}$ are normalization constants. In the low and high energy limits, we have respectively $F\left(E \ll E_{p}\right) \propto t^{\alpha_{0}-\alpha_{p} \beta_{1}}$ and $F\left(E \gg E_{p}\right) \propto t^{\alpha_{0}-\alpha_{p} \beta_{2}}$. Joint $\mathrm{XRT}$ and BAT observations fit with a Band model give $\alpha_{p}=-1.6, \beta_{1} \approx-0.13$, and $F_{B A T} \sim F\left(E \gg E_{p}\right) \propto t^{-2.0}$ (Toma et al. 2007). There is considerable uncertainty in $\beta_{2}$, so we do not fix its value, but rather try several values in the observed range. Choosing $\beta_{2}=-1.9$ and $\alpha_{0}=1$, we have $F\left(E \ll E_{p}\right) \propto t^{0.8}$, giving a model that roughly reproduces the time behavior and relative flux in the XRT and BAT bands (see Figure 2.6). These choices are consistent with observations of the early XRT light curve, which show $F_{X R T} \propto t^{0.7}$. 


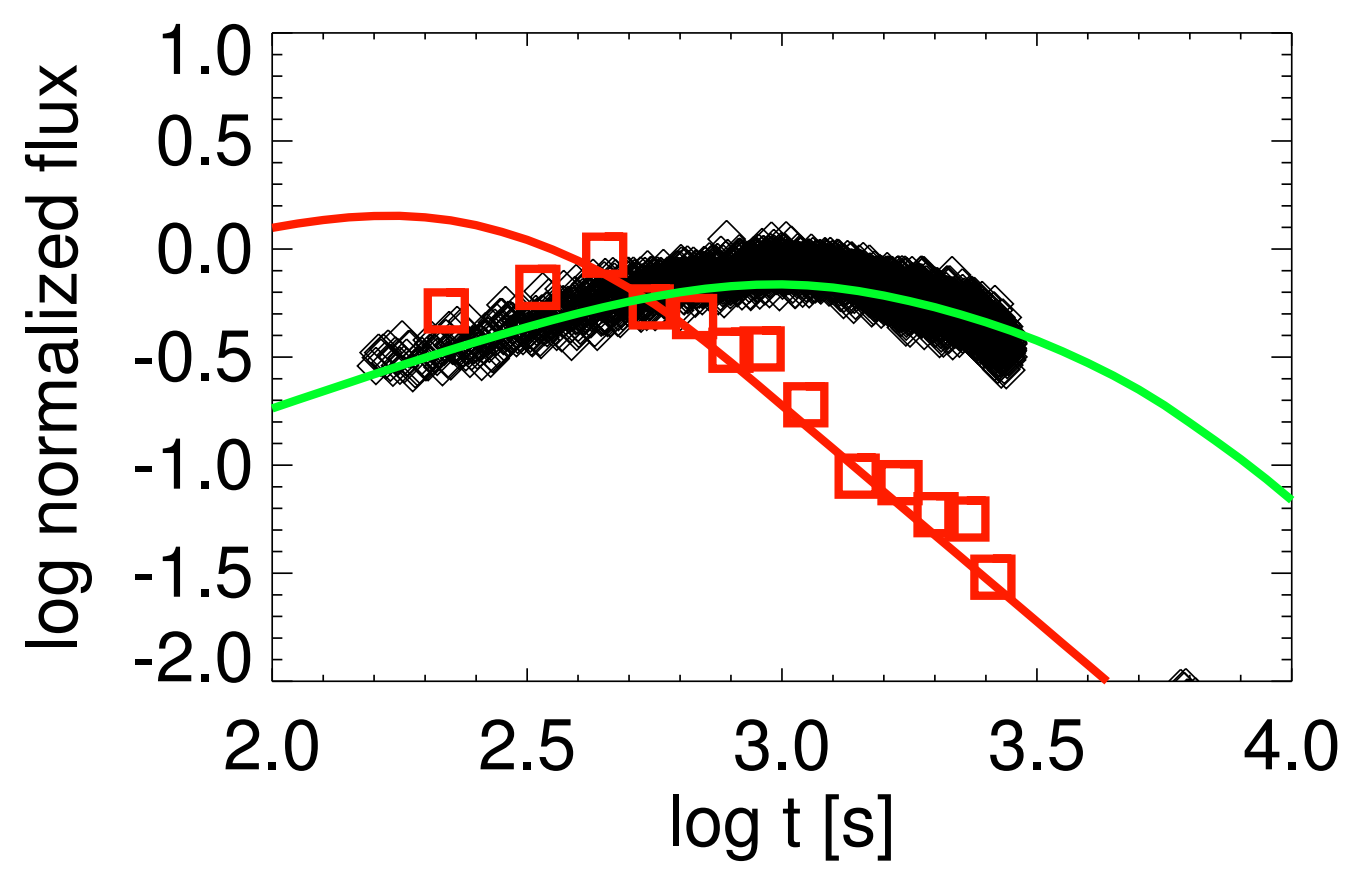

Fig. 2.6. - Light curves obtained using a Band spectrum with time-varying parameters $E_{p} \propto t^{-1.6}$ and $F_{0} \propto t^{1.0}$, and constant spectral indices $\beta_{1}=-0.13$ and $\beta_{2}=-1.9$. Model light curves in the $0.3-10 \mathrm{keV}$ band (green) and the $15-150 \mathrm{keV}$ band (red) are compared with XRT data from Campana et al. (2006) (black diamonds) and BAT data from Toma et al. (2007) (red squares). Both light curves are fit well after $400 \mathrm{~s}$, although the $15-150 \mathrm{keV}$ flux is too high at early times.

\section{Towards an inverse Compton model}

The presence of a thermal component in the XRT band with a timescale and luminosity comparable to the nonthermal component motivates consideration of an inverse Compton (IC) mechanism for the prompt emission. Before investigating physical situations that might lead to strong IC upscattering, we first discuss some features generic to IC models for GRB 060218. For now, we assume only that some hot electrons are present in the burst environment, but place no condition on the location of these electrons or the mechanism of their acceleration. 
First, if the primary photon source is approximately monochromatic (as is the case for the observed constant-temperature thermal component), then the quickly decaying peak energy implies that the scattering electrons are rapidly decelerating in the comoving frame. If the source frequency is $\nu_{0} \sim k_{B} T_{0}$ in the observer frame, and the electrons dominating the radiation have comoving Lorentz factor $\gamma_{p}$, then the IC component will peak at $E_{p} \sim \gamma_{p}^{2} k T_{0}$. This implies $\gamma_{p} \propto t^{-0.8}$, since $E_{p} \propto t^{-1.6}$ (Toma et al. 2007) and $T_{0}$ is approximately constant (Campana et al. 2006). Suppose the electrons are accelerated to characteristic Lorentz factor $\gamma_{m}$, and can cool to Lorentz factor $\gamma_{c}$ in time $t$. If cooling is slow (i.e., $\gamma_{m}>\gamma_{c}$ ), then $\gamma_{p} \sim \gamma_{m}$. On the other hand, if cooling is rapid $\left(\gamma_{c}>\gamma_{m}\right)$, then $\gamma_{p} \sim \gamma_{c}$ when $p<3$, or $\gamma_{p} \sim \gamma_{m}$ when $p>3$, because the $\nu F_{\nu}$ spectrum goes as $\nu^{(3-p) / 2}$ between $\nu_{m} \equiv \gamma_{m}^{2} k T_{0}$ and $\nu_{c} \equiv \gamma_{c}^{2} k T_{0}$ (Sari et al. 1998). $E_{p}$ evolves from $\sim 40 \mathrm{keV}$ at first detection to $\sim 1 \mathrm{keV}$ at $2000 \mathrm{~s}$ (Kaneko et al. 2007), and $k_{B} T_{0} \simeq 0.17 \mathrm{keV}$ for most times, implying that $\gamma_{p}$ varies from $\sim 15$ to 2 throughout the prompt phase.

Second, the scattering medium is at most moderately optically thick, i.e. the electron scattering optical depth is $\tau_{e} \lesssim 1$. This follows from the observation of distinct thermal and nonthermal components, since when $\tau_{e} \gg 1$, essentially all of the photons undergo multiple scatterings, resulting in a single nonthermal peak. A rough estimate of $\tau_{e}$ can be made by comparing the number of thermal and nonthermal photons. The thermal component, with peak luminosity $L_{t h} \sim 1 \times 10^{46} \mathrm{erg} \mathrm{s}^{-1}$ and timescale $t_{t h} \sim 3000 \mathrm{~s}$, carries $N_{t h} \sim L_{t h} t_{t h} / k T_{0} \sim 1 \times 10^{59}$ photons. The nonthermal component has $L_{n t} \sim 3 \times 10^{46} \mathrm{erg} \mathrm{s}^{-1}$ at peak, duration $t_{n t} \sim 1000 \mathrm{~s}$, and peak energy $E_{p} \sim 1 \mathrm{keV}$ near maximum light, and therefore contains $N_{n t} \sim 2 \times 10^{58}$ photons. This implies $\sim 1 / 6$ of thermal photons are scattered by electrons with $\gamma_{e} \simeq \gamma_{p}$, i.e. $\tau_{e} \sim 0.2$. Note that $N_{t h} \propto L_{t h} t \propto t^{1.8}$ according to our model for thermal emission in Section 
2.4.1, while $N_{n t} \propto F_{0} t \propto t^{2}$ according to the Band function model described earlier in this section. Hence $\tau_{e} \propto t^{0.2}$ is approximately constant in time in the simplest description.

Third, the nonthermal luminosity exceeds the thermal luminosity throughout most of the evolution. This implies that the Compton parameter $y \sim \tau_{e}\left\langle\gamma_{e}^{2}\right\rangle \gtrsim 1$. $\left\langle\gamma_{e}^{2}\right\rangle$ represents the average gain per scattering. Because $\tau_{e} \lesssim 1$, we require $\left\langle\gamma_{e}^{2}\right\rangle \gtrsim \tau_{e}^{-1} \gtrsim$ 1 to get $y \gtrsim 1$, suggesting at least mildly relativistic electrons. Since the total nonthermal luminosity is $L_{n t} \sim \tau_{e}\left\langle\gamma_{e}^{2}\right\rangle L_{t h}$, we can write $y \sim L_{n t} / L_{t h}$. Near maximum light, when the nonthermal component peaks in the XRT band, we can estimate $y$ directly: at $1000 \mathrm{~s}$ we have $L_{n t} / L_{t h} \sim y \sim 6$, and at $3000 \mathrm{~s}$ we have $L_{n t} \approx L_{t h}$ and $y \sim 1$. In our simple Band function description, $y \propto E_{p} F_{0} / L_{t h} \propto t^{-1.4}$, consistent with the values above.

Finally, the prompt spectrum holds information about the distribution of scattering electrons. For electrons distributed as a power law in energy with index $p$, the spectral slope above $\nu_{c}$ and $\nu_{m}$ is given by $F_{\nu} \propto \nu^{-p / 2}$. The high energy spectrum in GRB 060218 has typical index $\beta_{2} \approx-1.5$, but $\beta_{2}$ varies from -3 to -1 , implying $p$ is in the range $2-6$ with typical value $p \approx 3$. While most GRBs have $p$ values closer to $2, p=3$ is not outside of the observed spread in $p$ values (Panaitescu \& Kumar 2002).

In summary, any IC model for GRB 060218 should be in the limit of moderately small scattering optical depth but appreciable energy gain, so that the thermal component carries most of the photons (i.e., $N_{n t} / N_{t h} \sim \tau_{e} \lesssim 1$ ), but the nonthermal component carries most of the energy (i.e., $E_{n t} / E_{t h} \sim y \gtrsim 1$ ). $\tau_{e}$ most likely varies slowly in time, while $y$ and $\gamma_{p}$ decrease rapidly. We now look at how well three different IC models satisfy these criteria. 


\section{The photospheric model}

In the past several years, prompt thermal X-rays have been inferred from spectral fits in a number of GRBs, prompting the investigation of Comptonized photospheric models for the primary radiation (for a recent review, see Pe'er 2015, and references therein). In this picture, some of the bulk kinetic energy is dissipated into leptons within the jet, perhaps by internal shocks or magnetic effects. Depending on the optical depth at which the dissipation occurs, different emergent spectra are possible. Numerical simulations by Chhotray \& Lazzati (2015) have shown that, for leptons initially distributed as a power law $N(E) \propto E^{-p}$ (as typically assumed for shock heating), the observed spectrum takes the form of a thermal component with a high-energy power-law tail for relatively low dissipation optical depth $\tau_{\text {diss }} \sim 0.01-0.1$. This type of spectrum is qualitatively similar to the observed spectrum of GRB 060218. If the dissipation optical depth is decreasing with time, then one expects the peak energy to continuously decrease and the nonthermal component to become relatively weaker until only the thermal component remains. This, too, is qualitatively consistent with GRB 060218, where the nonthermal component gradually fades, leaving a blackbodydominated spectrum by $\sim 7000 \mathrm{~s}$.

However, the photospheric view is not without its problems. For one, it is unclear how quickly $E_{p}$ decays. In cosmological GRBs, the peak of the nonthermal component and the temperature of the thermal component are observed to be correlated, i.e. $E_{p} \propto T_{0}^{\alpha_{T}}$ with $\alpha_{T}$ typically $1-2$, and $T_{0}$ typically evolves with time (Burgess et al. 2014). GRB 060218 does not obey this correlation, nor does it show evidence for an evolving temperature. Nevertheless, the photospheric model provides a reasonable framework to interpret the early spectrum in GRB 060218, and is deserving of further attention. More work is needed to understand subphotospheric emission, particularly 
in the low-luminosity and small Lorentz factor limit, and in the case where a dense envelope surrounds the progenitor star, but detailed photospheric modeling is beyond the scope of the the current paper.

\section{IC emission from external shocks in a long-lived jet}

A long-lived central engine not only serves as a possible source of strong photospheric emission, but also influences ejecta dynamics by driving shocks into the surrounding medium. Here, we investigate IC scattering of prompt thermal photons from relativistic electrons in an engine-sustained reverse shock as a source for the observed prompt nonthermal emission.

First, we argue against the forward shock (FS) as the predominant IC emission site, because in this case obtaining a rapid decline of both the peak energy and the high energy light curve is difficult. Let the density of circumstellar material (CSM) as a function of radius be $\rho \propto r^{-\alpha}$. A steep $E_{p}$ decline implies a rapid deceleration, which in turn suggests a flat CSM density profile so that the FS sweeps up mass more quickly. Yet, the optical depth through the shocked region $\tau \sim \kappa \rho r \propto r^{1-\alpha}$ actually increases with time when $\alpha<1$. Compounding this with the rising thermal luminosity $L_{t h} \propto t^{k}$, the peak spectral luminosity $\left(L_{\nu, \max } \propto L_{t h} \tau \propto t^{k} r^{1-\alpha}\right.$ in the optically thin case) rises sharply with time in a flat density distribution. Since the BAT band lies above the peak energy, the luminosity there scales as $L_{B A T} \propto L_{\nu, \max } \nu_{c}^{1 / 2} \nu_{m}^{(p-1) / 2}$ (Sari et al. 1998). Whether the peak is due to $\nu_{c}$ or $\nu_{m}$, the rising $L_{\nu, \max }$ makes it difficult to ever obtain a BAT flux that declines faster than the peak energy in the case where the FS dominates emission.

This problem is alleviated by considering the reverse shock (RS) as the emission site instead. An immediate question is how to attain a declining peak energy at 
the RS, since generally this shock would go to higher Lorentz factor (in the fluid frame) as the flow is decelerated. As it turns out, a long-lived central engine can help in this regard. In the limit where the engine deposits mass into the RS more quickly than the FS sweeps mass from the CSM, the dynamics differ markedly from the typical GRB case. To illustrate this, consider ejecta with mass $M_{e j}$ and Lorentz factor $\gamma_{e j}$ interacting with a cold CSM. Previous authors (e.g. Sari \& Piran 1995) have shown that the outflow undergoes a dynamical transition when the mass swept by the forward shock, $M_{s w}$, becomes equal to $\sim M_{e j} / \gamma_{e j}$. When $M_{s w} \ll M_{e j} / \gamma_{e j}$ the shocked shell at the ejecta-CSM interface coasts with a constant Lorentz factor $\gamma_{s h} \approx \gamma_{e j}$, and when $M_{s w} \gg M_{e j} / \gamma_{e j}, \gamma_{s h}$ evolves with time. In the typical GRB case, where there is no continued mass or energy input from the central engine, $M_{e j}$ is constant while $M_{s w}$ grows over time; the system begins in the coasting state, and the shell starts to decelerate once $M_{s w}$ becomes sufficiently large. However, for continuous mass ejection, $M_{e j} \propto \int \dot{M}_{j} d t$ also increases with time. The dynamics will be altered if $M_{e j}$ grows faster than $M_{s w}$, which is possible for a steep CSM density gradient. In that limit $M_{s w}>M_{e j} / \gamma_{e j}$ initially, and the shell accelerates, eventually reaching a terminal Lorentz factor $\gamma_{s h} \approx \gamma_{e j}$ once $M_{s w} \ll M_{e j} / \gamma_{e j}$. In this scenario, the RS decelerates steadily in the contact discontinuity frame. Therefore, $E_{p} \propto \nu_{m}$ falls off quickly if the emission comes from a rapid-cooling reverse shock. In a steep density gradient, $\tau \propto r^{1-\alpha}$ also decreases with time, making it possible for $L_{B A T}$ to decline faster than $E_{p}$ if the emission comes from the RS.

While this rough example serves to illustrate the benefit of prolonged engine activity, calculating the XRT and BAT light curves requires a more thorough model. For an engine-driven outflow the resulting forward-reverse shock system can be divided into four regions - the cold CSM, the shocked CSM, the shocked outflow, and the 
unchecked outflow - which we label with subscripts 1 to 4, respectively. Regions 2 and 3 are separated by a contact discontinuity. Taking a constant adiabatic index of $4 / 3$, and assuming that the bulk Lorentz factor and internal energy in regions 2 and 3 do not change much between the shock and contact discontinuity, the dynamics of this system are governed by the equations (Sari \& Piran 1995):

$$
\begin{gathered}
f \equiv \bar{\rho}_{4}\left(R_{r s}\right) / \rho_{1}\left(R_{f s}\right), \\
f=\frac{\left(4 \gamma_{2}+3\right)\left(\gamma_{2}-1\right)}{\left(4 \bar{\gamma}_{3}+3\right)\left(\bar{\gamma}_{3}-1\right)}, \\
\bar{\gamma}_{3}=\gamma \gamma_{2}\left(1-\beta \beta_{2}\right),
\end{gathered}
$$

and

$$
R_{f s} \approx R_{r s} \approx \frac{\beta_{2} c t}{1-\beta_{2}},
$$

where $R_{f s}$ is the radius of the FS and $R_{r s}$ is the radius of the RS. These equations are valid in both the ultra-relativistic and mildly relativistic limit, but break down as the forward shock becomes non-relativistic since in that case $R_{r s} \ll R_{f s}$.

To close the system of equations (2.20)-(2.23), expressions for the densities $\rho_{1}$ and $\bar{\rho}_{4}$ as functions of radius $r$ are needed. The outer wind density we parameterize by

$$
\rho_{1}=5 \times 10^{11} A_{*} r^{-2}\left(r / R_{e x t}\right)^{2-\alpha} \mathrm{g} \mathrm{cm}^{-3} .
$$

Here, $A_{*}$ is a parameter scaled to a pre-explosion mass-loss rate of $\dot{M}_{\text {wind }}=10^{-5} M_{\odot} \mathrm{yr}^{-1}$ and velocity $v_{\text {wind }}=1000 \mathrm{~km} \mathrm{~s}^{-1}$. While $A_{*}=1$ is typical for Wolf-Rayet progenitors, a star with an extended envelope could have a different mass loss history. $\alpha$ determines the slope of the CSM density profile, with $\alpha=2$ corresponding to the usual wind profile. For a given $A_{*}$, winds with different $\alpha$ are scaled to the same density at 
$R_{\text {ext }}$. The density of the inner wind is given by

$$
\bar{\rho}_{4}=\frac{1}{4 \pi r^{2} \beta \gamma} \dot{M}_{i s o}\left(t_{e m i t}\right)
$$

with $\dot{M}_{\text {iso }}$ given by equation (2.1). The time $t_{\text {emit }}=\left(\beta-\beta_{2}\right) t / \beta\left(1-\beta_{2}\right)$ takes into account the delay between the arrival of photons and matter from the central engine at the reverse shock. Note that our model is only valid when $\alpha<3$ (i.e., when the swept CSM mass is not negligible) and $k>-2$ (i.e., when the energy input from the central engine is not negligible).

We solved numerically the system of equations (2.20)-(2.25) to determine the dynamical variables. For reference, we also present analytical solutions of these equations in the limit $\gamma \gg 1$ in Appendix A. Once $\gamma_{2}, \bar{\gamma}_{3}$ (or $\bar{\beta}_{3}$ ), and $\mathrm{R}$ are known, the spectral parameters $\nu_{m}, \nu_{c}$, and $L_{\nu, \max }$ for the forward- and reverse-shocked regions can be determined using the standard theory, as outlined in Appendix B, where we give analytical expressions for the maximum spectral power $L_{\nu, \max }$ and break frequencies $\nu_{c}$ and $\nu_{m}$. In calculating the spectrum, we approximate the thermal photons as monochromatic with frequency $\sim 3 k T_{0}$. We take the blackbody temperature to be $0.14 \mathrm{keV}$ as in Kaneko et al. (2007), as we find this gives a better fit than the higher value inferred by Campana et al. (2006). The nonthermal spectrum is taken to have a power-law form with breaks at $\nu_{m}$ and $\nu_{c}$, and the BAT and XRT light curves are determined by integrating the spectrum over $15-150 \mathrm{keV}$ and $0.3-10 \mathrm{keV}$, respectively. Our best fitting models have $p>3$, so in all cases discussed here $E_{p} \simeq h \nu_{m}$. The free parameters of our models are $\alpha, p, A_{*}, \gamma$, and the fractions of postshock energy $\epsilon_{e 2}$ and $\epsilon_{e 3}$ going into relativistic electrons in the FS and RS respectively. We fix $k$, $L_{0}$, and $\xi$ to the values inferred from the observed thermal component, as in Section 2.4.1. 
We calculated $L_{B A T}, L_{X R T}$, and $E_{p}$ over a range of parameter space. In order to match the observed slope of $E_{p}$ and $L_{B A T}$, we fixed $\alpha$ to 2.7; for other values of $\alpha$, the fit for these observables is typically worse, although the fit to $L_{X R T}$ is sometimes improved. We varied $p$ from $3.0-4.0$ in steps of $0.1, \log A_{*}$ from $2.0-5.0$ in steps of $0.0125, \gamma_{e}$ from $6.0-12.0$ in steps of 0.025 , and $\log \epsilon_{e 3}$ from -3.5 to -1.5 in steps of 0.025. In no case were we able to fit the spectrum reasonably with FS emission, so rather than vary $\epsilon_{e 2}$, we fit the light curves with the sum of thermal emission and nonthermal RS emission, and then calculate an upper limit on $\epsilon_{e 2}$ by assuming the FS contributes less than $30 \%$ of the flux in XRT and BAT. In addition, we place an upper limit on $\epsilon_{B}$ by demanding that the comoving energy density $\left(u_{\text {rad }}\right)$ of thermal radiation at the $\mathrm{RS}$ be higher than the comoving energy density in magnetic fields $\left(u_{B}\right)$. For each model, we calculated the reduced chi-squared $\chi^{2}$ via a joint fit to the observed XRT luminosity from Campana et al. (2006) and the BAT luminosity and peak energy measured by Toma et al. (2007). We do not include the XRT data after $4000 \mathrm{~s}$ in the fit.

In some cases - particularly when $A_{*}$ is rather large - the optical depth of the shocked regions exceeds unity. Our model, which assumes single scattering, is not valid in that case. Thus, we discard models with high optical depth, keeping only models that become optically thin prior to $300 \mathrm{~s}$. After that cut is applied, our bestfitting model has $p=3.8, A_{*}=4900, \gamma=10.8$, and $\epsilon_{e 3}=6.0 \times 10^{-3}$. This model is shown in Figure 2.7. We find that the FS does not contribute substantially to the emission when $\epsilon_{e 2} \lesssim 0.05 \epsilon_{e 3} . u_{\text {rad }} / u_{B}>1$ at all times in this model if $\epsilon_{B}<2 \times 10^{-5}$. If we relax the condition to $u_{\text {rad }} / u_{B}>1$ only after $300 \mathrm{~s}$, then $\epsilon_{B} \lesssim 10^{-4}$. While the low upper limit on $\epsilon_{B}$ is somewhat troubling, we note that other authors (e.g., Fan et al. 2006; Barniol Duran et al. 2015) have also found a low value of $\epsilon_{B}$ compared to 
$\epsilon_{e}$ in GRB 060218.
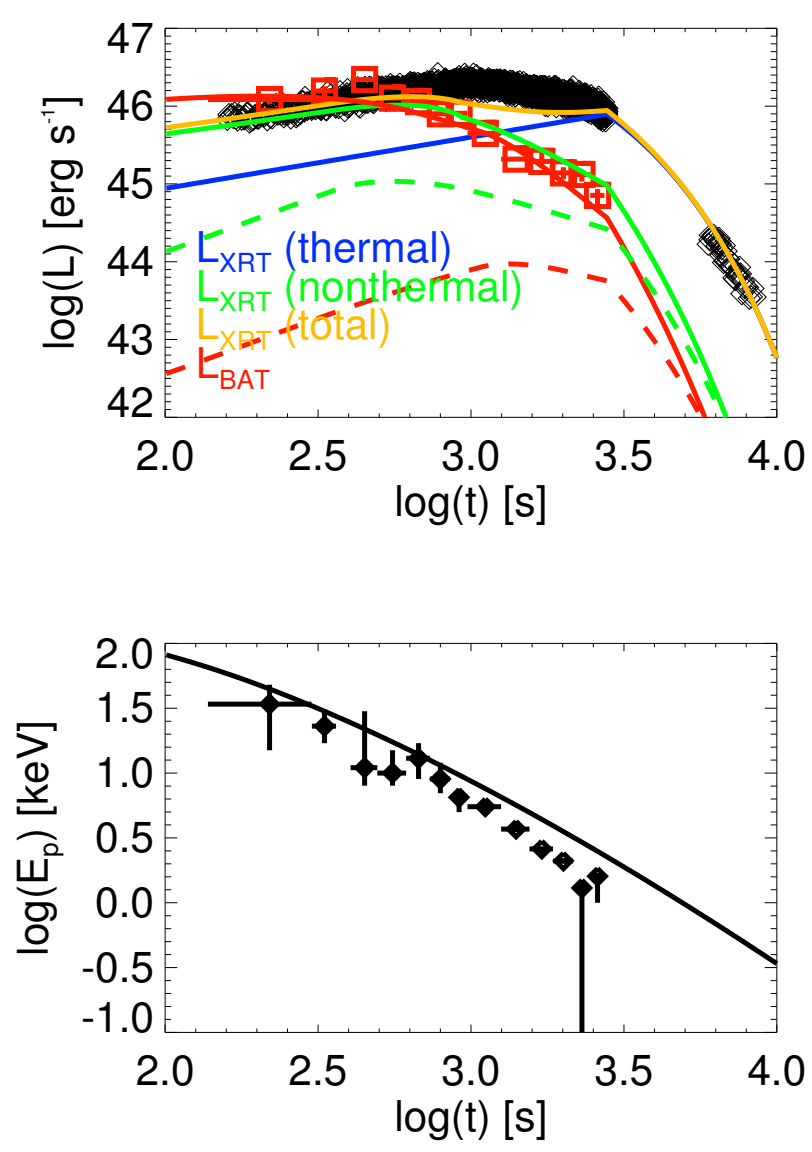

Fig. 2.7.- Top: XRT (0.3-10 keV) and BAT (15-150 keV) light curves for our reverse shock IC model. When fixing $k=0.66, \xi=1, L_{0,46}=1$, and $\alpha=2.7$, the best-fitting parameters are $\epsilon_{e 3}=6.0 \times 10^{-3}, \gamma=10.8, A_{*}=4900$, and $p=3.8$ The thermal, nonthermal, and total XRT luminosities are shown in blue, green, and orange respectively, while the BAT luminosity is drawn in red. Dashed curves show the contribution of the forward shock to the BAT (red) and XRT (green) light curves, assuming $\epsilon_{e 2} / \epsilon_{e 3}=0.05$. The black diamonds are XRT data from Campana et al. (2006), and the red squares are BAT data from Toma et al. (2007). Bottom: The peak energy in our model, as compared to data from Toma et al. (2007).

While this model can plausibly fit the light curves, it cannot explain the lowfrequency spectral shape: for $\nu_{c}<\nu<\nu_{m}$, we have $F_{\nu} \propto \nu^{-1 / 2}$, steeper than the observed spectrum $F_{\nu} \propto \nu^{-0.1}$. However, we note that Toma et al. (2007) found a 
different spectral shape at low energies, $F_{\nu} \propto \nu^{-0.4}$, when using a cut-off power-law to fit the data instead of a Band function, so the observed $\beta_{1}$ seems to depend in part on the assumed spectral model. We also find a high value of $p \simeq 3.8$ that, while roughly consistent with the observed value of Toma et al. (2007), is large compared to the value in typical GRBs (Panaitescu \& Kumar 2002). An additional issue with our model is that it slightly underpredicts the XRT flux near peak by up to a factor of 2 , and slightly overestimates the peak energy. Furthermore, our model is only onedimensional, and it does not take into any effect of collimation or sideways expansion of the jet.

Despite these issues and the crudeness of the model, the reverse shock IC interpretation does a reasonable job of capturing the basic behavior of the light curves, and it has some attractive features. Notably, of all the models we consider, this model is the only one that provides a natural explanation for the steep decline of $E_{p}$ and $L_{B A T}$. Additionally, this type of emission is expected when a long-lived, dissipative jet is present, and should therefore contribute to the emission on some level (although, IC emission from the RS only dominates the contribution from external shocks under certain circumstances, as described above). However, if other lepton populations (e.g., those excited by internal shocks or other dissipation in the jet interior) also strongly contribute to the emission, the external shock emission may not be observed.

Having an independent estimate for the outflow Lorentz factor allows us to break the degeneracy of our radio model discussed in Sections 2.4.5 and 2.4.6, by calculating $E_{\text {iso }}$ directly. Assuming $t_{L}$ corresponds to the turn-off time of the engine, we find $M_{\text {iso }} \simeq 5 \times 10^{-3} M_{\odot}$ and $E_{\text {iso }} \simeq 9 \times 10^{52} \mathrm{ergs}$ via equations (2.4) and (2.5). Thus, the high energy, high Lorentz factor radio model is preferred. The low upper limits on $\epsilon_{B}$ and $\epsilon_{e}$ in this section are consistent with the ranges inferred from the radio (see 
discussion at the end of Section 2.4.6). The jet is cold and radiates inefficiently, with $\eta_{\gamma} \simeq 7 \times 10^{-4}$. Applying $\theta_{0} \gtrsim \gamma^{-1}$, we find that the true jet energy is $E_{j} \gtrsim 4 \times 10^{50}$ ergs. These results have interesting implications when compared to standard GRBs. $E_{i s o}$ and $E_{j}$ fall within the range typical for cosmological bursts (Piran 2004), suggesting that the total kinetic energy released in GRB 060218 is not unusual, although it is released over a longer time. The main factor that distinguishes GRB 060218 from the bulk of observed GRBs is therefore its radiative efficiency: whereas most bursts have $E_{\gamma, \text { iso }} \approx E_{\text {iso }}$ (Piran 2004), our model for GRB 060218 predicts $E_{\gamma, \text { iso }} \ll E_{\text {iso }}$. This fact is closely linked to the low values we deduced for the $\epsilon$ parameters, which may perhaps be related to the lower bulk Lorentz factor or the long engine lifetime. For now, this is only speculation, but the possibility that standard GRBs are a corner case where the radiative efficiency is high (due, perhaps, to a higher Lorentz factor or a more abrupt deposition of kinetic energy), while most collapsar events go unobserved because of a much lower radiative efficiency, is intriguing.

In addition, we can deduce some properties of the CSM near the progenitor from the inferred value of $A_{*}$. If it extends in to $R_{e x t}$, the wind is optically thick to electron scattering, with total optical depth $\tau_{w}=\int_{R_{e x t}}^{\infty} \rho_{1} \kappa d r \approx 30$. However, because of the steep density gradient, the high optical depth is due mostly to material very close to $R_{\text {ext }}$. In fact, the shock radius in our model is $R \approx 5 \times 10^{13} \mathrm{~cm}$ at the time of first observation; our model does not constrain the wind density at radii less than this. The optical depth of the wind is small compared to the envelope optical depth, so the addition of such a wind does not affect the optical model discussed in Section 2.4.3. This wind cannot be the origin of the high value of $N_{H}$, however, as the absorbing column through the wind changes as the shock propagates outward, while the observed $N_{H}$ is constant. 
The extent of the wind is not known, but the equipartition radius $R_{N}=1.3 \times$ $10^{16} \mathrm{~cm}$ at day 5 gives an upper limit, since as discussed in Section 2.4.5 the radio observations imply a constant density CSM. The total mass of the wind is therefore $M_{w}<\int_{R_{e x t}}^{R_{N}} 4 \pi r^{2} \rho_{1} d r \simeq 4 \times 10^{-3} M_{\odot}$. This is comparable to the isotropic mass of the jet, so it is possible that the jet undergoes some deceleration while sweeping the outer layers of the envelope. In addition, even though the terminal Lorentz factor is $\gamma_{2} \approx \gamma=10.8$ in our model, the transition to the coasting state is quite gradual: we find $\gamma_{2}$ only reaches $\approx 5$ by the end of the prompt phase at $t_{L}$. It therefore seems plausible that the jet could decelerate to $\beta \gamma \sim 1$ by day 5 , as implied by radio observations. We stress, however, that there is still some tension in producing the flat radio light curve with a mildly relativistic outflow.

After the source of thermal photons fades away, the emission from external shocks will be dominated by synchrotron radiation. Since the overall SED appears incompatible with a single synchrotron spectrum, this component should not overwhelm the optical emission and dust echo afterglow emission observed at the same time. Since the jet begins to decelerate shortly after $t_{L}$ in our model, the synchrotron emission peaks near $t_{L}$. At that time we find that the critical synchrotron frequencies are $\nu_{m} \simeq 6 \times 10^{-9} \mathrm{~Hz}$ and $\nu_{c} \simeq 4 \times 10^{12} \mathrm{~Hz}$; both are far below the optical band. We calculate a peak synchrotron $\nu F_{\nu}$ flux of $5 \times 10^{-15} \mathrm{erg} \mathrm{cm}^{-2} \mathrm{~s}^{-1} 2 \times 10^{-17} \mathrm{erg} \mathrm{cm}^{-2} \mathrm{~s}^{-1}$, respectively at $5 \times 10^{14} \mathrm{~Hz}$ and $1 \mathrm{keV}$. This is far below the observed $\nu F_{\nu}$, which is $\sim 10^{-11} \mathrm{erg} \mathrm{cm}^{-2} \mathrm{~s}^{-1}$ in both the X-ray and optical bands Toma et al. (2007). The main reason synchrotron emission from the jet is so weak compared to cooling envelope emission and dust scattering of the prompt light is the low values of $\epsilon_{e}$ and $\epsilon_{B}$. This answers the question posed at the end of Section 2.4.4, suggesting that the primary reason a dust echo is observed in GRB 060218 is because of low values of the 
microphysical parameters.

\section{Other models for jet emission}

Here we briefly consider some other possibilities for the prompt emission, but each is problematic. Thus, we prefer an inverse Compton interpretation for the prompt emission in GRB 060218.

Synchrotron emission from external and/or internal shocks is also expected for relativistic jets. The standard FS synchrotron model, with constant external density and no continuous energy injection (i.e., $\alpha=0$ and $k=-2$ ), gives $E_{p} \propto \nu_{m} \propto t^{-3 / 2}$ and $F\left(E \gg E_{p}\right) \propto t^{(2-3 p) / 4}$ in the rapid cooling limit (Sari et al. 1998). When $p \simeq 3$, this gives a time behavior similar to the observed one. However, the peak energy in this case is much too low to explain observations, even for unphysically high explosion energies.

Internal shock models, in which ejecta shocked by the collision of successive enginelaunched shells radiate via synchrotron, remain a prominent model for the prompt radiation in cosmological GRBs (Piran 2004). This picture provides a natural interpretation of the high degree of variability in GRB light curves, as the many shell collisions give rise to multiple peaks. GRB 060218, with its smooth, single-peaked light curve, may therefore be hard to explain in an internal shock context, unless an additional mechanism acts to smooth out the light curve. It is unclear, as well, how the presence of an extended envelope could affect the internal shock signature.

In some cases, steep decays in the prompt GRB light curve have been attributed to a kinematical effect, wherein the observer continues to see emission from highlatitude parts of the curved emission region after the prompt emission process ends. This phenomenon, known as the curvature effect, leads to fainter and softer emission 
over time because of relativistic beaming. Toma et al. (2007) already investigated curvature effects for GRB 060218, and showed that the simultaneous steep decay of the peak energy and high energy light curve is inconsistent with this interpretation.

Finally, we compare the expected shock breakout signal in our model with the observed prompt emission. The shock breakout has two components: one associated with the breakout of the jet from the envelope, and one with the breakout of the SN shock. The breakout signal can be characterized by an breakout mass $M_{b r}$ and velocity $v_{b r}$ (or Lorentz factor $\gamma_{b r}$ ), and the envelope radius $R_{e x t}$ and opacity $\kappa_{e x t}$. The breakout occurs at an optical depth $\tau_{b r} \sim c / v_{b r} \sim \kappa_{e x t} M_{b r} / R_{e x t}^{2}$. Assuming order-unity radiative efficiency, the breakout luminosity can be estimated as $L_{b r} \sim$ $E_{b r} / t_{b r}$, where $E_{b r}$ and $t_{b r}$ are respectively the breakout energy and timescale. In the relativistic (jet) breakout case, we have $E_{b r} \sim \gamma_{b r} M_{b r} c^{2}, t_{b r} \sim R_{e x t} / 2 \gamma_{b r}^{2} c$, and $\tau_{b r} \sim$ $c / v_{b r} \sim 1$ so that $M_{b r} \sim R_{e x t}^{2} / \kappa_{e x t}$. The resulting luminosity is $L_{b r} \sim 2 c^{3} R_{e x t} \gamma_{b r}^{3} / \kappa_{e x t}$, or $L_{b r} \sim 3 \times 10^{45} \mathrm{erg} \mathrm{s}^{-1} R_{e x t, 13} \kappa_{e x t, 0.2}^{-1} \gamma_{b r}^{3}$, where $R_{e x t}$ has been scaled to $10^{13} \mathrm{~cm}$ and $\kappa_{\text {ext }}$ to $0.2 \mathrm{~cm}^{2} \mathrm{~g}^{-1}$. If $\gamma_{b r}$ is large, it is possible that the jet breakout signal could be brighter than the observed luminosity, which is $\sim 10^{46} \mathrm{erg} \mathrm{s}^{-1}$ at early times; however, this signal would also be short $(\sim 1-10 \mathrm{~s})$ and could have easily been missed in GRB 060218. The interaction of the cocoon with circumstellar gas may also produce a transient; however, because the cocoon's energy and velocity post-breakout are small compared to the energy and velocity of the fast SN ejecta (see discussion in Section 2.4.6), any signal from circumstellar interaction should be dominated by the SN.

In the non-relativistic case appropriate for SN shock breakout, we have instead $E_{b r} \sim M_{b r} v_{b r}^{2} / 2, t_{b r} \sim R_{e x t} / c$, and $\tau_{b r} \sim c / v_{b r}$ so that $M_{b r} \sim R_{e x t}^{2} c / \kappa_{e x t} v_{b r}$. The luminosity in this case is $L_{b r} \sim c^{2} v_{b r} R_{e x t} / 2 \kappa_{e x t}$, or $L_{b r} \sim 7 \times 10^{44} \mathrm{erg} \mathrm{s}^{-1} R_{e x t, 13} \kappa_{e x t, 0.2}^{-1} \beta_{b r}$, with $\beta_{b r}=v_{b r} / c$. Even if $\beta_{b r} \approx 1$, the SN breakout luminosity in our model is modest 
compared to the observed X-ray signal.

\subsection{Discussion}

We have presented a model for the peculiar GRB 060218 in which the prompt X-ray emission arises from a low-power jet and the early optical emission is powered by fast SN ejecta interacting with a low-mass circumstellar envelope. Our picture has some features in common with the recent model of Nakar (2015), where the prompt X-ray and optical emission is produced by a choked jet interacting with a circumstellar envelope. In both cases, a jet is needed to decouple the mildly relativistic outflow from the SN, and an extended envelope of similar mass $\left(\sim 0.01 M_{\odot}\right)$ and radius $\left(\sim 100 R_{\odot}\right)$ is inferred. Both models provide a reasonable explanation for the radio afterglow flux, although Nakar's model has an advantage in explaining the shallow slope of the light curve. Neither model can account for the X-ray afterglow through external shock synchrotron radiation alone. There are several key differences between the models, however. We differ on the jet properties (we suggest a low-power, longduration jet, whereas Nakar uses a more typical GRB jet), the origin of the prompt X-rays (we prefer a dissipative jet and some Compton scattering process, whereas Nakar posits shock breakout), and the power source for the cooling envelope emission seen in the optical band (we suggest that it is driven by the underlying SN, whereas Nakar proposes a smothered jet explosion). A detailed discussion of the strengths and weaknesses of each model is therefore warranted.

An advantage of Nakar's model is that the luminosity and timescale of the jet take on typical GRB values. In addition, shock interaction naturally produces a smooth, single peaked light curve in X-rays, as observed (Nakar \& Sari 2012). This model also helps to explain the lack of a jet break in the radio, since the jet outflow becomes 
quasi-spherical before leaving the envelope. A wind-like CSM profile is also inferred for afterglows powered by shock breakout (Barniol Duran et al. 2015), which is expected for a WR star progenitor. On the other hand, the high value $(\sim 50 \mathrm{keV})$ and slow decay $\left(\propto t^{-(0.5-1)}\right)$ of the prompt peak energy that one expects in the relativistic shock breakout scenario of Nakar \& Sari (2012) seem hard to reconcile with direct measurements of the peak energy that show it declines steeply as $t^{-1.6}$ and with a value of $\sim$ a few keV throughout most of the prompt phase (Toma et al. 2007). (However, as discussed in Section 2.3, mildly relativistic effects and deviation from spherical symmetry may rectify this issue.) The fact that the prompt optical and X-ray emission are observed simultaneously, and that they each evolve smoothly from the earliest observation, also seems hard to interpret in a shock interaction model where the observed radiation evolves from a nonequilibrium state toward thermal equilibrium. A better understanding of the expected X-ray signal from mildly relativistic shocks in low-mass envelopes is needed to determine whether these problems can be resolved. The origin of the prompt thermal X-ray component is also unclear in the choked jet model, since the inferred photospheric radius is considerably smaller than the envelope radius.

Our low-luminosity jet model comes with its own merits and drawbacks. A jet origin for the prompt X-rays and $\gamma$-rays places GRB 060218 at the low-luminosity, long-duration end of a continuum of GRB processes. In this unified picture, similarities to cosmological bursts (such as satisfying the Amati and lag-luminosity correlations) are perhaps not surprising, although as in typical GRBs the physical origin of these correlations is not well understood. nonetheless, these coincidences are not easily accounted for in the shock interaction view. Furthermore, the presence of a thermal component is expected for a dissipative jet, and having a separate origin 
for the prompt X-ray and optical emission removes problems with the X-ray to optical evolution. However, the low-power jet interpretation inherits one usual problem with jet models, namely that the prompt emission mechanism in relativistic jets is still not well understood. Also, since a low-luminosity jet stays collimated while it drills through the circumstellar envelope, our model requires the jet to become nonrelativistic in the CSM, which may be difficult unless some additional mass close to the star helps to decelerate the jet. While we infer a wind-like CSM at small radii where the prompt X-rays are emitted, we find that a uniform circumburst density is needed beyond $10^{16} \mathrm{~cm}$ where the radio is emitted. This is contrary to usual expectations for a WR progenitor. Finally, we require an unusually long-lived, low-power central engine, the origin of which is unclear.

This last point deserves more discussion. A shortcoming of our model, as with many engine driven models, is the need to prescribe unknown properties of the central engine. A simple parametrization glosses over many of the finer details of compact object formation and jet launching, the physics of which are not yet fully understood. In particular, producing a long-duration, low-luminosity engine from a nascent black hole presents problems: Aloy et al. (2005) have shown in their black hole simulations that a minimum jet luminosity of $\sim 10^{49} \mathrm{erg} \mathrm{s}^{-1}$ is needed to overcome the ram pressure of accreting material, and black hole-driven engines tend to operate on timescales much shorter than $\sim 10^{3}$ seconds. However, the SN might clear away infalling material, thus allowing a lower luminosity jet to propagate. It is unclear whether the formation of a neutron star (or magnetar) could drive the type of mildly relativistic outflow we require, but the longer timescales involved are more consistent with the long-lasting prompt emission observed (for further discussion, see Toma et al. 2007). Magnetar-powered scenarios are particularly intriguing in light of the recent result 
of Greiner et al. (2015), who claim the detection of magnetar-driven superluminous SN associated with the ultra-long GRB 111209A. Here, we only aim to show that a low-luminosity outflow, if present, can explain many features of the prompt thermal and nonthermal emission. Note that several other bursts, such as GRB 130925A (Evans et al. 2014) and the ultra-long bursts discussed by Levan et al. (2014), may also require long-lived central engines, so this problem is not unique to GRB 060218.

The lack of variability in the light curves of GRB 060218 and GRB 100316D merits further investigation, as well. If the typical GRB variability originates from relativistic turbulence, then the smooth light curves observed in some LLGRBs could be ascribed to the lack of highly relativistic material (Narayan \& Kumar 2009). Even in the absence of relativistic effects, a light jet lifting heavier external material would give rise to Rayleigh-Taylor instabilities that may induce light curve fluctuations. This could be circumvented by, e.g., a Poynting-flux dominated jet, but a matter-dominated jet is required to produce the prompt nonthermal X-rays through IC processes. The smooth light curve also constrains the degree of clumpiness which can be present in the CSM. Detailed numerical simulations will be needed to characterize the amount of variability expected from a mildly relativistic jet as it penetrates the star and envelope, breaks out from the envelope's edge, and sweeps the surrounding CSM.

We note that, because our model involves an on-axis jet, we cannot appeal to geometric effects to increase the rate of 060218-like events. However, our model does imply a unique, non-standard progenitor different from the usual high-mass WR stars thought to give rise to most cosmological LGRBs. Thus, our explanation for the high rate of LLGRBs is simply that LLGRB progenitors are intrinsically 10-100 times more common. Assuming that the presence of a long-lived, low-luminosity jet is also somehow tied to the progenitor structure, such jets might also be more common than 
ultra-relativistic, short-lived ones, but we are biased against observing them due to their low power. In the model of Nakar (2015), the progenitor is again different from the standard one, but the prompt emission is roughly isotropic as well, so that the higher event rate is due to some combination of geometric and intrinsic effects.

An essential constraint on our model is that the beaming-corrected rate of GRB 060218-like events should not exceed the rate of broad-lined Type Ibc SNe. Guetta \& Della Valle (2007) estimate a SN Ibc rate of $2 \times 10^{4} \mathrm{Gpc}^{-3} \mathrm{yr}^{-1}$, with the rate of broadlined events being $\sim 7 \%$ of this, or $\sim 10^{3} \mathrm{Gpc}^{-3} \mathrm{yr}^{-1}$. The rate of events like GRB 060218, 230 $-190 \mathrm{Gpc}^{-3} \mathrm{yr}^{-1}$ (Soderberg et al. 2006), is still uncertain, but could be as low as $40 \mathrm{Gpc}^{-3} \mathrm{yr}^{-1}$. The rate estimate of Soderberg et al. (2006) included 1998bw (which likely has a different origin), and only took into account one year of Swift observations. These factors could combine to lower the rate by perhaps a factor of a few, but this reduction in rate is offset somewhat by including the similar burst GRB 100316D. Our theory, which includes a wide jet with beaming factor $f_{b} \sim 10-100$, is marginally compatible with the lower end of the range of rates given in Soderberg et al. (2006). If subsequent observations determine a higher event rate (indicating that GRB 060218-like events are nearly isotropic), it would pose a severe problem to our model.

It may be possible to construct a "hybrid" model that retains some of the best features of both our model and Nakar's. This speculative model is depicted in Figure 2.8. Suppose that the central engine switches off while the jet is traversing the envelope (as in Nakar's model), but let the envelope mass be smaller (as in our model) so that the outflow decelerates significantly and expands sideways somewhat, but does not have time to sphericize before breaking out. The explosion then breaks out aspherically, with shock breakout emission expected from near the poles. If dissipation continues 
to occur after the cessation of engine activity, a thermal X-ray component might also be observable once the ejecta clear out. After breakout, the ejecta expand into the low-density CSM, eventually producing the radio synchrotron emission as electrons are accelerated by the external shocks. Since the outflow expands preferentially into the CSM post-breakout, a quasi-toroidal envelope remnant is left behind, which is shocked by the fast SN ejecta and then emits the prompt optical emission as it cools. The X-ray afterglow is produced by dust scattering, as in Section 2.4.4. As with our model, this scenario gives a possible explanation for the thermal emission, and since the optical and X-ray are decoupled there are no concerns with the X-ray to optical spectral evolution. Yet, as with Nakar's model, this case generates a smooth prompt X-ray light curve via shock breakout, and more easily explains the radio because the initial outflow is wider than a jet. However, the expected signal from an aspherical shock breakout in the relativistic limit has not been calculated in detail, which is an important caveat.

Moving past the prompt emission, we have also shown that a dust echo model gives a reasonable fit to the X-ray afterglow light curve and spectral index evolution. The dust echo model used only an empirical fit to the prompt light curve and spectrum, and therefore is insensitive to the mechanism of prompt emission. Moreover, the scattering angle from the dust grains, $\theta_{d} \approx\left(2 c t / R_{d}\right)^{1 / 2} \sim 1^{\circ}\left(R_{d} / 30 \mathrm{pc}\right)^{-1 / 2}(t / 10 \text { days })^{1 / 2}$, is small, so the echo emission depends only on the prompt radiation roughly along the observer's line of sight. Thus, the dust echo interpretation applies equally well whether the prompt emission originates from a low-luminosity jet or from shocked gas. If the reason for the small radiated energy in bursts like GRB 060218 is small values of $\epsilon_{e}$ and $\epsilon_{B}$, as our model suggests, then dust echo type afterglows should commonly accompany this class of bursts, because the synchrotron emission from ex- 


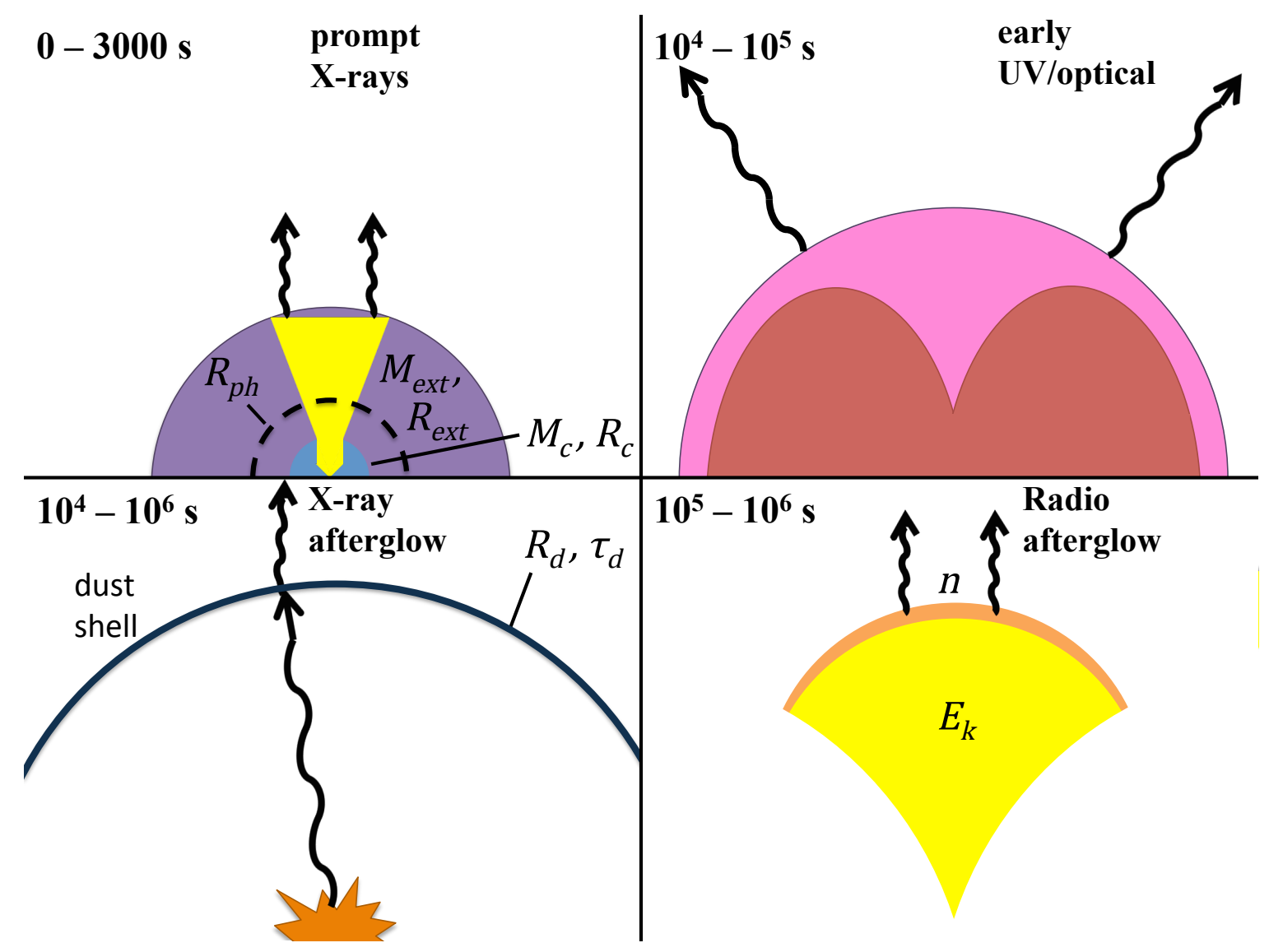

Fig. 2.8. - A hybrid model for the prompt emission. Upper left: A jet is launched with short timescale compared to the envelope breakout time, as in Nakar (2015). However, the jet does not have time to become quasi-spherical before breaking out; it undergoes significant deceleration, and possibly a small degree of lateral spreading, but the explosion breaks out primarily in the forward direction, leaving the envelope mostly intact. Thermal emission could be observed, e.g. from the walls of the jet cavity, once material clears out along the line of sight. Upper right: As in our model, the fast SN ejecta heat the remaining envelope, which cools through optical radiation. Lower left: The X-ray afterglow is produced from dust scattering, as described in Section 2.4.4. Lower right: The radio afterglow comes from a non-relativistic, quasispherical blast wave. Because the ejecta are already decelerated to $\beta \gamma \sim 1$ by the envelope, a spherical flow is more readily achieved than in our jet breakout model.

ternal shocks will be weak. So far, this is borne out by observations, as the afterglow GRB 100316D is also consistent with a dust echo. Why the synchrotron efficiency is poor, and whether this is related somehow to the long burst duration, remains to be 
worked out.

If our picture for GRB 060218 is correct, one would expect to observe broad-lined Type Ic SNe with accompanying mildly relativistic radio afterglows, but without a prompt X-ray component, when viewing GRB 060218-like events off-axis. The global rate of such events would be some 10-100 times greater than the on-axis rate, assuming wide opening angles in the range of $\sim 10-30$ degrees. Such events might be uncovered by radio follow-up of Type Ic SNe. Future survey projects such as the Large Synoptic Survey Telescope should detect more Type Ic SNe with a doublepeaked signature of cooling envelope emission, expanding the number of potential interesting targets for radio follow-up.

Whether or not the long prompt emission is tied to the presence of a circumstellar envelope is an interesting open question. Clearly, this is so for the model of Nakar (2015). For our model, though, the prompt X-ray and optical emission have different origins, so it may be possible to observe an X-ray signal akin to that of GRB 060218 with no prompt optical counterpart. (On the other hand, if the envelope plays a crucial role in jet dissipation, it may still be needed.) The high- $T_{90}$, high-variability light curves of ultra-long GRBs do seem to suggest the possibility of intrinsically long-lasting jet emission. Interestingly, several ultra-long bursts (e.g., GRB 101225A, GRB 111209A, and GRB 121027A) also show an early optical peak that may be consistent with shock cooling (Levan et al. 2014). In other ultra-long GRBs (e.g., GRB 130925A and GRB 090417B), no optical light was detected, but the presence of early optical emission cannot be ruled out due to the high extinction to those events (Holland et al. 2010; Evans et al. 2014). Overall, prompt optical emission is observed more often than not in very long bursts, hinting at one of two possibilities: either the engine duration is long because a circumstellar envelope is present, or a 
circumstellar envelope is present because the progenitors of long-duration engines also tend to have circumstellar envelopes. We note that, because the jet presumably breaks out successfully in ultra-long GRBs, any optical emission due to extended envelope interaction in ultra-long bursts must be powered by the supernova, and not by a choked jet. This topic is of considerable theoretical interest going forward.

Regardless of whether objects like GRB 060218 are powered by a jet or a shocked envelope, circumstellar interaction clearly has a role to play in explaining these unusual LLGRBs. Both our model and the Nakar (2015) model can be taken as further indirect evidence for the existence of a dense environment immediately surrounding the progenitor star, indicative of strong pre-explosion mass loss or binary evolution. The mechanism driving this mass loss is unclear, but possibilities include late unstable nuclear burning (Smith \& Arnett 2014), gravity wave-driven mass loss (Quataert \& Shiode 2012), or common envelope evolution (Thöne et al. 2011; Chevalier 2012). Alternately, the circumstellar envelope could arise from a stripped binary scenario as in Type IIb SNe. We emphasize that the progenitor's pre-explosion history is a crucial factor in determining the observed radiation's characteristics, and that this theme applies broadly to many transients including Type Ia-CSM, Type IIn, and Type IIb SNe. Understanding the late phases of intermediate- to high-mass stellar evolution will play a critical role as our ability to detect transient phenomena continues to evolve.

\subsection{Conclusions}

We have presented a comprehensive model for the unique LLGRB GRB 060218 that provides reasonable explanations for each of its features. The model includes a peculiar engine-driven jet with a low luminosity $\left(L_{i s o} \sim 3 \times 10^{49} \mathrm{erg} \mathrm{s}^{-1}\right)$ and a long 
duration $\left(t_{j} \sim 3000 \mathrm{~s}\right)$, properties that we suggest are related to a non-standard progenitor. We have shown that, if the jet dissipates some modest fraction of its kinetic energy into thermal radiation, Comptonization of seed thermal photons by hot electrons can explain features of the prompt spectrum, light curve, and peak energy evolution. We investigated different emission sites for the IC process, and found that scattering from electrons in the reverse-shocked gas can roughly account for the prompt X-ray light curve and peak energy decay, if the fraction of energy put into magnetic fields and into electrons in the forward-shocked gas is small. Scattering from a nonthermal electron population within a dissipative jet outflow also remains a possibility for the prompt emission. Scattering from forward shock electrons can be ruled out, as the light curves and peak energy cannot be reproduced in this case. We also argued against a synchrotron origin for the prompt emission.

We analyzed constraints on the jet properties from the prompt thermal emission, the radio afterglow, and dynamical considerations. There exists a region of parameter space that can fit both the radio afterglow and the prompt thermal emission without violating other constraints, although there is considerable degeneracy that prevents precise determination of the parameters. The early thermal emission and the late-time radio afterglow can be explained either by a cold jet with relatively high energy and Lorentz factor and relatively little postshock energy in electrons and magnetic fields, or by a hot jet with lower energy and Lorentz factor and standard choices for $\epsilon_{e}$ and $\epsilon_{B}$. Our IC model for the prompt emission breaks this degeneracy, strongly preferring the former scenario. We derived the jet parameters $E_{k} \simeq 4 \times 10^{50} \mathrm{ergs}, \gamma \simeq 11$, and $\theta_{0} \simeq 0.1$, and find that the immediate circumstellar environment has a density profile of $\rho_{1} \propto r^{-2.7}$ and wind parameter $A_{*} \simeq 4900$. The inferred microphysical parameters of the reverse shock are $\epsilon_{e 3} \simeq 6 \times 10^{-3}$ and $p=3.8$. Combining the radio and prompt 
X-ray models, we constrained the magnetic parameter to $10^{-5.5} \lesssim \epsilon_{B} \lesssim 10^{-4}$ and the electron energy fraction in the forward shock to $10^{-5.5} \lesssim \epsilon_{e 2} \lesssim 10^{-3.5}$. Radio observations constrain the density at $r>10^{16} \mathrm{~cm}$ to be constant, with $n \sim 10^{3.5}$ $10^{5.5} \mathrm{~cm}^{-3}$ depending on the values of $\epsilon_{e 2}$ and $\epsilon_{B}$. However, there is some concern that the outflow will not have time to sphericize prior to the radio observations, which makes the shallow radio light curve difficult to interpret. Our results suggest that GRB 060218 may be an engine-driven event that has the same kinetic energy coupled to relativistic ejecta as in typical GRBs, but radiates very inefficiently in comparison. This result has interesting implications considering the high volumetric rate of LLGRBs.

We have shown as well that the early peak in optical/UV can be powered by interaction of the fast outer SN layers with a low-mass extended envelope surrounding the progenitor star. With the SN parameters inferred for SN 2006aj, and the luminosity and blackbody radius implied by the measured host extinction, we derive the envelope parameters $M_{\text {ext }} \approx 4 \times 10^{-3} M_{\odot}$ and $R_{\text {ext }} \approx 9 \times 10^{12} \mathrm{~cm}$. SN 2006aj is perhaps the best case so far of a double-peaked light curve characterized by cooling envelope emission, as described by Nakar \& Piro (2014).

We also tested the idea that the unusual X-ray afterglow in GRB 060218 is a dust echo of the prompt emission, as suggested by Margutti et al. (2015) to explain the extremely soft afterglow spectrum. Using the available prompt emission data as an input, we modeled the expected dust echo emission from a shell of dust at $R_{d}$, with scattering optical depth $\tau_{d}$ at $1 \mathrm{keV}$. Assuming dust grains distributed uniformly in size from a minimum radius $a_{-}=0.005 \mu \mathrm{m}$ to a maximum radius $a_{+}=0.25 \mu \mathrm{m}$, we found that $\tau_{d}=0.007$ and $R_{d} \simeq 28$ pc gave a good fit to the afterglow light curve and the spectral index evolution. (However, we stressed that there is considerable 
uncertainty in $R_{d}$, as it is sensitive to certain poorly constrained dust properties.) Because the echo emission does not depend on the prompt emission mechanism, this result is robust, making GRB 060218 quite a convincing case for a dust echo. The echo model implies only a modest amount of dust consistent with the dust content of the ISM. That the dust echo dominates over the usual synchrotron afterglow can be explained in this case by a low value of $\epsilon_{e}$ and $\epsilon_{B}$, consistent with our radio estimates and prompt X-ray modeling. We compared our results for GRB 060218 to the other bursts with soft afterglow spectra identified by Margutti et al. (2015), and found that two distinct classes of echo-dominated afterglows are indicated: one requiring a typical amount of dust (like GRB 060218), and one requiring an unusually high amount of dust (like GRB 130925A).

We conclude by noting that our understanding of the class of low-luminosity, ultralong GRBs with smooth light curves is severely hindered by the the small sample size - presently, GRB 060218 and GRB 100316D are the only constituent members of this class. In addition, because GRB 100316D lacks a detection of prompt optical emission or clear-cut evidence for prompt thermal emission, we are unable to draw any firm conclusions about it in our model. More observations of this unique class of objects is needed to settle questions about the prompt emission mechanism and the transition from beamed to spherical outflow, and to better constrain the properties of the progenitor, envelope, jet, and CSM. With the rates estimated by Soderberg et al. (2006), Swift should turn up a new burst in this class every several years or so. In the meantime, advancing our theoretical understanding of shock-envelope interaction, the emission mechanism in relativistic jets, and the propagation of jets in complex circumstellar environments can furnish testable predictions for the next observed event. 


\section{Acknowledgments}

We thank R. Barniol-Duran and B. Morsony for helpful discussions, and E. Nakar

for valuable comments on the manuscript. This research was supported in part by NASA Grant NNX12AF90G. 


\section{Chapter 3}

Numerical Simulations of

Relativistic Jets in Extended

Circumstellar Envelopes 


\begin{abstract}
Motivated by recent suggestions of low-mass, extended envelopes surrounding the progenitors of Type Ic supernovae, we perform adaptive mesh refinement hydrodynamical simulations of GRB jets in extended circumstellar media. We derive a straightforward analytical picture to predict the conditions under which a jet is choked by the extended envelope, and apply it to interpret the numerical results. Our results show that in the envelope, the jet-cocoon system is generally more collimated than analytically predicted at times after the engine turns off. In contrast to what has been assumed by some authors, we find that the outflow is still far from spherical at breakout, for a wide range of relevant parameters. However, even though the flow is not spherical, most of the energy is in the cocoon, so that the outflow sphericizes on a short timescale after envelope breakout. This differs markedly from the standard case of a bare star, where the jet does become spherical if choked, and the outflow after breakout remains beamed for some time. These results have important implications both for the event rate of low-luminosity gamma-ray bursts, and for the origin of their prompt emission.
\end{abstract}

\title{
3.1 Introduction
}

The early evolution of a supernova $(\mathrm{SN})$ is shaped by the immediate circumstellar environment of its progenitor star, and rapid advances in SN detection and followup have enabled astronomers to observationally probe a progenitor's pre-explosion surroundings. Recent observations of a number of core-collapse SNe show an early peak that points to the presence of an extended, low-mass envelope surrounding the progenitor star (Nakar \& Piro 2014). In Chapter 2.4.3, we discussed the application of this model to the broad-lined Type Ic SN 2006aj. However, this is not the only 
type of SNe whose early emission is best explained by a cooling envelope. Recently, extended envelopes have been inferred for several other SN subtypes as well, including Type Ib/c SNe (Drout et al. 2016), standard Type Ic SNe (Taddia et al. 2016), and superluminous Type Ic SNe (Nicholl \& Smartt 2016).

It is most interesting that broad-lined Type Ic SNe are among the classes for which extended envelopes are indicated, since these are thought to be the progenitors not only of LLGRBs, but of standard GRBs as well. GRBs are understood to be powered by a relativistic jet that is launched when the progenitor star's core collapses to a black hole (e.g, MacFadyen \& Woosley 1999), but the presence of sufficiently dense circumstellar material can have a profound effect on the propagation of this jet. For example, if the operating time of the jet is sufficiently long to escape the progenitor star, but not the extended envelope, the envelope can suffocate the jet leading to a quasi-spherical, mildly relativistic "choked jet" explosion that produces a transient in $\gamma$-rays and X-rays when it breaks out (Nakar 2015). On the other hand, if the jet's luminosity is low and its duration is long, it can be collimated by the extended envelope; in this case a weak GRB is produced after the jet drills through the envelope, and later interaction of the SN with the remaining envelope material powers an early optical signal (Irwin \& Chevalier 2016). Both of these interpretations have been put forward to explain the peculiarly long and faint GRB 060218 and its associated SN 2006aj (Nakar 2015; Irwin \& Chevalier 2016).

Many authors have studied the propagation of the jet through the host star (see Section 1.2), as well as the behavior and ultimate sphericization of the jet in a lowdensity circumstellar environment (see Section 2.4.5). The choking of jets by their host stars has also been studied by several authors. For example, Couch et al. (2008) studied the propagation of jets through red giant progenitors and found that a SN-like 
quasi-spherical explosion could be produced if the ratio of thermal energy to kinetic energy in the jet is large. Lazzati et al. (2012) investigated how engine duration affects jet propagation in WR stars (i.e., typical GRB progenitors). They found that the jet could produce a successful GRB; a weak GRB with a relativistic SN resembling SN 1998bw; a mildly relativistic SN showing some signs of engine activity like SN 2009bb; or a non-relativistic explosion indistinguishable from a typical Type Ic SN, depending on whether the engine timescale is respectively longer, about the same, shorter, or much shorter than the time to breakout of the star. They suggested that the central engine timescale is the primary driver of the diversity of Type Ic SNe seen in nature.

The idea of choked jets has even received considerable attention from the neutrino astrophysics community. The idea is that as the jet stalls and its Lorentz factor drops, the optical depth to photons can increase to the point that it becomes more efficient to radiate by neutrinos, making jets choked within the star a promising target for neutrino detection (Mészáros \& Waxman 2001; Horiuchi \& Ando 2008; Bartos et al. 2012; Tamborra \& Ando 2016). Senno et al. (2016) considered an analytical model for neutrino production in the case where the choking is done by an extended circumstellar envelope rather than by the host star. In the process, they derived an analytical condition distinguishing choked from successful jets, which agrees with the results of Nakar (2015) and our analytical model discussed below.

Despite much active research on jet propagation through the host star, to date a detailed numerical study of the dynamics of a jet interacting with a low-mass, extended circumstellar envelope has not been carried out. This work differs from past studies of jet propagation because we include such an envelope. The process of jet choking is expected to be different in this case than in the case where the jet 
is choked within the progenitor star, because the jet-cocoon system is in a different dynamical regime for standard parameters (see Section 1.2). The main difference is that, in the star, the head of the jet is highly non-relativistic, and as a result the bulk of the jet material catches up to the head and flows into the cocoon shortly after the engine shuts off. In an extended envelope, on the other hand, the jet head tends to be relativistic, and energy flows into the cocoon much more slowly.

In this chapter, we present the results of numerical simulations of GRB jets in progenitor stars with extended envelopes. We focus on envelopes with properties similar to those inferred in Section 2.4.3 and in Nakar (2015) (as this is the best observed case of a broad-lined Type Ic SN with an early optical peak), and on jets consistent with those that produce standard GRBs. We explore central dynamical questions such as the effect of an envelope on the morphology of the jet at breakout (as compared to the case of a bare star) and the conditions under which an initially collimated jet is choked by the envelope. In addition, we test the analytical predictions of Nakar (2015), and assess the viability of his model as an explanation for longduration, low-luminosity GRBs such as GRB 060218.

In Section 3.2, we discuss the code used and the simulation setup. We then consider an analytical framework for interpreting results in Section 3.3. In Section 3.4, we present the results of four different simulations. We discuss ramifications in Section 3.5, and summarize our conclusions in Section 3.6.

\subsection{Methods}

We carried out our simulations with the relativistic ENZO (RENZO) code (Wang et al. 2008). Built on the foundation of the adaptive mesh refinement hydrodynamics code ENZO (Bryan et al. 2014), RENZO is a fully relativistic magnetohydrodynamics 
code that includes an accurate transrelativistic equation of state (EOS) (Mignone et al. 2005), making it well-suited to applications involving low Lorentz-factor flows. The specific EOS adopted here is model TM from Mignone et al. (2005), which interpolates between the non-relativistic and relativistic regimes to recover the ideal gas EOS in either limit. Our simulation setup is akin to previous studies of GRB jet propagation (e.g., Morsony et al. 2007; Mizuta et al. 2006; Mizuta \& Ioka 2013): a jet parameterized by luminosity, opening angle, and Lorentz factor is injected at a small initial radius into a model progenitor system. In our case, this system includes a central star with radius $\sim 10^{11} \mathrm{~cm}$, a dense envelope extending to $\sim 10^{13} \mathrm{~cm}$, and a low density circumstellar medium (CSM) beyond that. We track the jet as it penetrates the progenitor star, breaks out into the surrounding extended envelope, and then traverses the envelope and emerges into the CSM. The simulations are 2D and axisymmetric, with reflecting boundaries at the jet axis and equatorial plane and outflow boundaries otherwise. The grid used is Cartesian; we refer to positions within the grid using cylindrical coordinates $(r, z)$. We define $R \equiv\left(r^{2}+z^{2}\right)^{1 / 2}$ as the radial distance from the origin. Consistent with other authors (e.g., Morsony et al. 2007), cells within the adaptive mesh are flagged for further refinement if the normalized second derivative within the cell exceeds 0.8. For further discussion of the second derivative refinement method in ENZO, see Section 3.6 in Bryan et al. (2014). A Courant-Friedrichs-Lewy number of 0.4 is used for all simulations (i.e., the time step in a cell of size $\Delta$ never exceeds $0.4 \Delta / c$ ).

\subsubsection{Grid setup}

We have modified the RENZO code to include a feature from a newer ENZO version that allows the user to vary the maximum allowed level of refinement over the grid. 
This is especially beneficial in our case because of the large dynamic range range of our problem: we are interested in length scales ranging from a few times the size of the circumstellar envelope $\left(\sim 10^{13} \mathrm{~cm}\right)$ to the width of the collimated jet at the injection site $\left(\sim 10^{8} \mathrm{~cm}\right.$; see Section 3.2.2). Because the highest levels of refinement are necessary only very close to the jet axis, this modification significantly improves runtime. Fig. 3.1 shows the maximum level of refinement as a function of grid position. We choose the refinement regions such that the spine of the jet is always resolved (spanned by at least $\sim 5$ grid cells), and the resolution is sufficient to capture the forward and reverse shocks. We use the same grid setup for all simulations. The top level grid is a square with side length $R_{g}=2.6 \times 10^{13} \mathrm{~cm}$. Each simulation runs for $1.1 R_{g} / c \approx 950 \mathrm{~s}$, which is sufficient time for the outflow to cross the outer grid boundary.

Compared to previous simulations (e.g. Zhang et al. 2003; Morsony et al. 2007; Mizuta \& Ioka 2013), our simulations have a lower resolution within the star. Essentially, we trade some resolution in the star for better resolution at the larger radii of interest to us (previous simulations have not included an extended envelope, so the total size of the grid was much smaller). As a result, we miss out on much of the substructure present in the cocoon, particularly the turbulence and vortices that form in the backflow of spent jet material. Nonetheless, we find roughly the same global properties (breakout time, cocoon shape, and so forth) as in past numerical and analytical studies, as we show in Section 3.4.1 below.

\subsubsection{Jet injection}

We inject the jet as a boundary condition on the lower edge of the grid. Following other recent simulations of collapsar jets (Mizuta et al. 2006; Mizuta \& Ioka 2013), we 


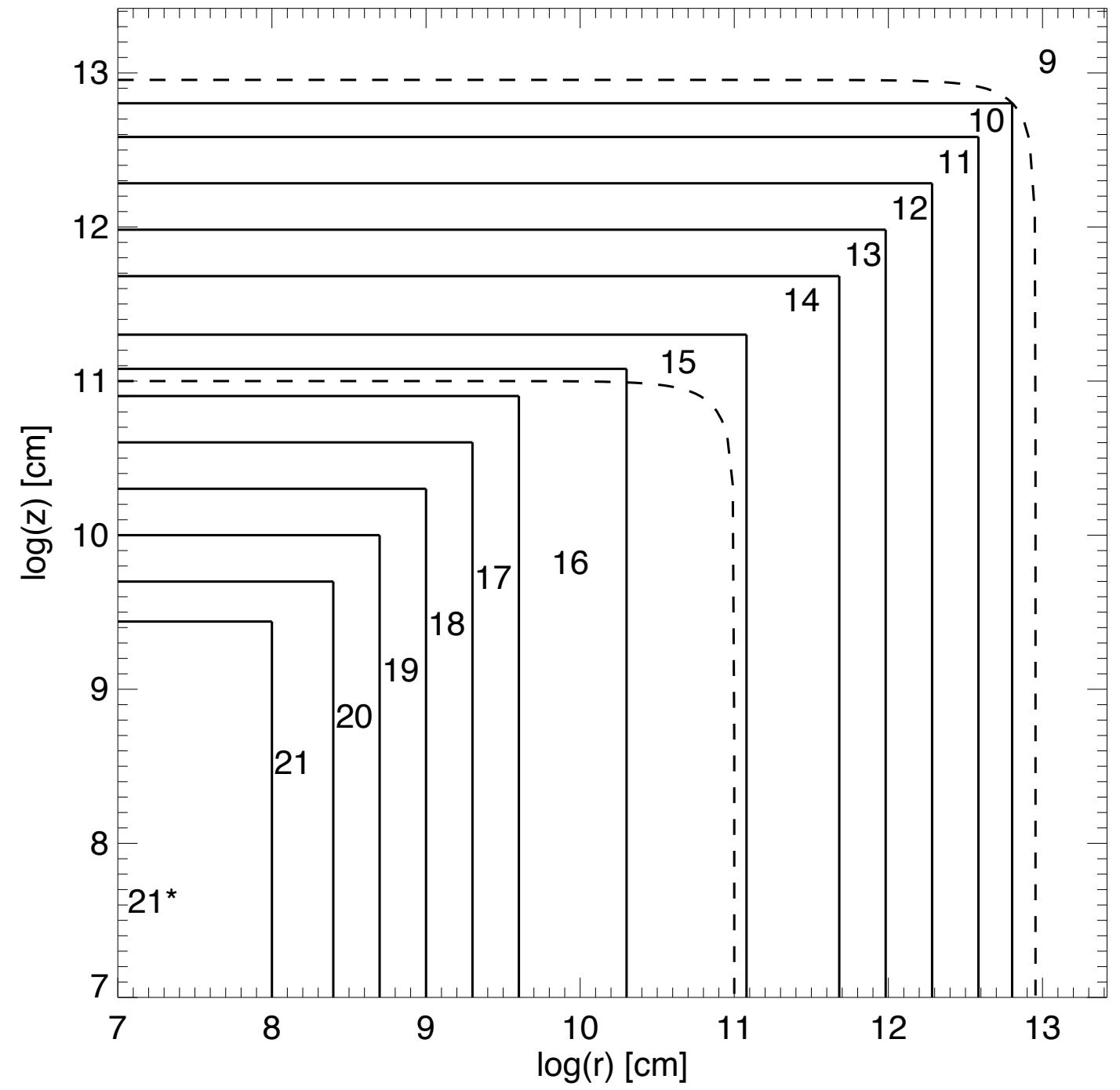

Fig. 3.1.- Maximum refinement level as a function of grid position. The numbers indicate how many times the base grid is allowed to be refined by a factor of 2 , i.e. the maximum resolution in a region marked with number $n$ is $R_{g} / 2^{n}$. The region marked as 21 is always refined to the maximum level to ensure that the jet is always resolved at small radii. The radii of the star and envelope are also shown as dashed lines.

inject the jet parallel to the $z$-axis. The lower boundary of the simulation is located at $z_{0}=2.56 \times 10^{9} \mathrm{~cm}$. In order to get the dynamics right, it is necessary for the width of the injected jet, $r_{0}$, to be less than the true width of a collimated jet at 
the injection site, $r_{j}$. Otherwise, the resulting outflow will be too wide and too slow; eventually, the solution will converge to the correct one, but the time for this to occur can be comparable to or longer than the breakout time from the star. This problem was discussed in detail by Mizuta \& Ioka (2013), who also provide a numerically calibrated expression for the jet width. Taking $z_{h}=z_{0}$ in their equation (24), along with the parameters for our model star described in Section 3.2.3, gives the time $t_{0}$ for the jet to reach the boundary at $z_{0}$. Then, substituting this time into their equation (25), we obtain

$$
r_{j} \simeq 9 \times 10^{7}\left(\frac{z_{0}}{2.5 \times 10^{9} \mathrm{~cm}}\right)^{2 / 3}\left(\frac{L_{j}}{10^{51} \mathrm{erg} \mathrm{cm}^{-1}}\right)^{1 / 6}\left(\frac{\rho_{0}}{1500 \mathrm{~g} \mathrm{~cm}^{-3}}\right)^{-1 / 6}\left(\frac{\theta_{0}}{0.2}\right)^{4 / 3}
$$

where $\rho_{0}$ is the stellar density at $z_{0}, L_{j}$ is the jet luminosity, and $\theta_{0}$ is the jet opening angle. The requirement $r_{0}<r_{j}$, and the need to place several grid cells across the jet, sets the smallest length scale in the problem and determines the necessary resolution. We use $r_{0}=7.5 \times 10^{7} \mathrm{~cm}$ and put 6 cells across the jet at the boundary, corresponding to a resolution of $1.25 \times 10^{7} \mathrm{~cm}$ at the highest refinement level. We find no significant difference in the dynamics within the star when $r_{0}$ is halved and an additional level of refinement is used; this verifies that our solution is indeed converged.

The choice of inputting the jet parallel to the grid axis reflects the physical fact that the jet is expected to become collimated well before it reaches the simulation boundary at $2.56 \times 10^{9} \mathrm{~cm}$. In this limit, the collimated jet is fully characterized at the injection site by three parameters: $L_{j}$, the collimated jet Lorentz factor $\Gamma_{1}$, and the ratio of internal to rest-mass energy in the collimated jet, $\epsilon_{1}$. (We use the subscript 1 to refer to conditions in the jet when it is collimated, and the subscript 0 when it is uncollimated.) Assuming the uncollimated jet is cold $\left(\epsilon_{0} \approx 0\right)$, it is described by $\Gamma_{0}$ and $\theta_{0}$ rather than $\Gamma_{1}$ and $\epsilon_{1}$. Bromberg et al. (2011) show that $\Gamma_{1} \sim \theta_{0}^{-1}$. Using 
this, we can derive an equation for $\epsilon_{1}$ in terms of $\Gamma_{0}$ and $\theta_{0}$ by considering that the quantities $\Gamma \rho$ (mass flux) and $h \Gamma^{2} \rho-\Gamma \rho-p$ (energy flux) are conserved upstream and downstream of the collimation shock ${ }^{1}$. Here, $\rho$ is the jet density, $p$ is the jet pressure, $h \equiv 1+4 p / \rho c^{2}=1+4 \epsilon / 3$ is the enthalpy, and an adiabatic index $\gamma_{a d}=4 / 3$ has been assumed. Substituting $\epsilon_{0}=0$, and using $\rho_{0} \Gamma_{0}=\rho_{1} \Gamma_{1}$ to eliminate $\rho_{1}$ in the energy conservation equation, we obtain

$$
\Gamma_{0}-1=\left(\Gamma_{1}-1\right)+\frac{1}{3}\left(4 \Gamma_{1}^{2}-1\right) \epsilon_{1} / \Gamma_{1}
$$

after some simplification. Then, substituting $\Gamma_{1} \sim \theta_{0}$, we get

$$
\epsilon_{1} \sim \frac{3\left(\Gamma_{0}-\theta_{0}^{-1}\right)}{\theta_{0}\left(4 \theta_{0}^{-2}-1\right)}
$$

We use a standard choice of jet parameters for all simulations: $L_{j}=10^{50} \mathrm{erg} \mathrm{s}^{-1}$, $\Gamma_{1}=5$, and $\epsilon_{1}=0.75$, corresponding to $\Gamma_{0} \sim 10$ and $\theta_{0} \sim 0.2$. We do, however, vary the engine activity timescale $t_{j}$. The values of $L_{j}$ and $\theta_{0}$ are consistent with typical measured values for GRB jets, and with the model of Nakar (2015). The value of $\Gamma_{0}$ is lower than in standard GRBs, which generally have $\Gamma_{0} \sim 100$. However, the dynamics of the jet cocoon system do not depend on $\Gamma_{0}$ except in the free expansion phase, which is never approached in our models until the jet breaks out of the envelope into the low-density CSM. The choice of a lower $\Gamma_{0}$ allows us to capture the basic physics while using a much lower resolution in the CSM region, since the width of the forward-shocked region during free expansion, $\sim R / \Gamma_{0}^{2}$, is 100 times larger than it would be for $\Gamma_{0}=100$. This is justified since the dynamics in the CSM are not of

\footnotetext{
${ }^{1} \mathrm{~A}$ more accurate treatment would use the relativistic shock jump conditions at the collimation shock, and thus equation (3.2) and equation (3.3) would also include a dependence on $\Gamma_{s}$, the collimation shock Lorentz factor. But, since the collimation shock is non-relativistic, $\Gamma_{s} \approx 1$, justifying the approximation used here.
} 
great interest in this study. As discussed in Chapter $2, \Gamma_{0} \sim 10$ may also be more relevant to LLGRBs.

It is desirable to consider also the case of much lower jet luminosities and longer engine timescales, to test out the Irwin \& Chevalier (2016) model discussed in Chapter 2. However, this comes with a few technical challenges. For $L_{j} \sim 3 \times 10^{47} \mathrm{erg} \mathrm{s}^{-1}$, the jet width $r_{j}$ at the injection site is decreased by a factor of $\sim 3$, so that 1-2 additional levels of refinement are required for the same total grid size. A worse problem is the long jet duration. Typically, the highest-refinement regions at small radii are only needed so long as the engine is active, and refinement can be reduced after the engine shuts off. When the engine duration is long, however, these fine grids must be retained for the whole simulation. Due to the combination of these effects, it is significantly more expensive to model long-lived, low-luminosity jets. Future work will study the dynamics in this case.

\subsubsection{Star and envelope models}

We create a model star with mass $M_{*}=2 M_{\odot}$ and radius $R_{*}=10^{11} \mathrm{~cm}$. Previous numerical studies have used a larger progenitor mass, but we adopt the lower mass indicated by observations of SN 2006aj (Mazzali et al. 2006), the supernova associated with GRB 060218. The stellar parameters do not change from model to model. The mass is based on observations of SN 2006aj, and is somewhat lower than the mass 10$16 M_{\odot}$ typically used in collapsar jet simulations. We use a simple power-law model for

the stellar density profile, $\rho \propto R^{-2.5}$, and a pressure $p(R)=K \rho^{4 / 3}$. This simple model approximates the outer parts of an $n=3$ polytrope; for the given mass and radius, an $n=3$ polytrope model give $K=6.1 \times 10^{14}$ in cgs units. We have $p / \rho c^{2} \ll 1$ everywhere in the star. Morsony et al. (2007) tested this simple power-law model 
against a more detailed stellar density profile calculated by Woosley \& Heger (2006) via stellar evolution modeling, and found only minor differences in the dynamics. The simple model has the advantage that it lends itself readily to analytical estimates, as well as being more straightforward to implement in RENZO.

Because we do not include gravity in our simulations, the star is not in hydrostatic equilibrium, and its outer edge expands at a slow rate. Over the course of the simulation, the star expands by several $10^{9} \mathrm{~cm}$. This has a negligible effect on the breakout time and the overall dynamics. The same is true for the extended envelope.

We model the extended envelope as a spherical region with mass $M_{\text {ext }}$, radius $R_{\text {ext }}$, and density distributed as $\rho \propto R^{-\alpha_{e x t}}$. The values of $M_{e x t}, R_{e x t}$, and $\alpha_{e x t}$ are varied between models. Beyond the envelope, we include a low-density CSM with constant density $n=100 \mathrm{~cm}^{-3}$, as inferred from radio observations of GRB 060218 (Soderberg et al. 2006). The pressure in the envelope and CSM is set to a negligibly low value, and has no effect on the dynamics.

\subsection{Analytical expectations}

In this section, we consider an analytical model for jet propagation in the envelope, and derive the conditions under which the jet is expected to be choked. For our purposes, a "choked" jet is one for which all of the energy in the jet flows into the cocoon prior to breaking out of the envelope. Nakar (2015) also hypothesized that a choked jet will produce a quasi-spherical explosion; we aim to test this assumption in our simulations. In order for the jet to be choked by the envelope, two conditions must be met:

1. The jet duration, $t_{j}$, must be longer than the time to break out of the star, $t_{b, *}$; otherwise, the jet will stall in the star instead. 
2. The last of the jet material must catch up to the head of the jet before the head reaches the surface of the envelope.

Here, we assume an envelope density profile $\rho_{\text {ext }}=A R^{-2}$, where $A$ is a constant. This simplifies the analytical calculations, since in this limit the speed of the jet head is constant while traversing the envelope. The dynamics in the envelope then depend on the parameter

$$
\xi=\frac{L_{j}}{A c^{3}}
$$

$\xi$ is basically the same as the parameter $\tilde{L}$ introduced in Section 1.2 , except the dependence on $\theta_{0}$ has been factored out. The reason for this choice will become clear shortly, when we consider the parameter space of $t_{j}$ versus $\xi$. When using $\xi$, the jet dynamical regimes are as follows: collimated and non-relativistic for $\xi \ll \theta_{0}^{4}$; collimated and relativistic for $\theta_{0}^{4} \ll \xi \ll \theta_{0}^{2 / 3}$; uncollimated and causal for $\theta_{0}^{2 / 3} \ll$ $\xi \ll 4 \theta_{0}^{-2}$; uncollimated and non-causal for $4 \theta_{0}^{-2} \ll \xi \ll 4 \theta_{0}^{2} \Gamma_{0}^{4}$; and free expansion for $\xi \gg 4 \theta_{0}^{2} \Gamma_{0}^{4}$.

Let $\beta_{h}$ and $\Gamma_{h}$ be the velocity and Lorentz factor of the jet head. In the usual case where the jet head is non-relativistic inside of the star, the breakout time is given by $t_{b, *} \simeq L_{j}^{-1 / 3} \rho_{*}^{1 / 3} R_{*}^{5 / 3} \theta_{0}^{4 / 3}$, where $\rho_{*}=\left(3-\alpha_{*}\right) M_{*} / 4 \pi R_{*}^{3}$ is the stellar density near $R_{*}$, and the stellar density profile is $\rho \propto R^{-\alpha_{*}}$ (Bromberg et al. 2011). (Note that, throughout this section, we ignore constants of order unity to ensure a smooth transition between dynamical regimes.) However, for our parameters we typically find that the jet head becomes mildly relativistic $\left(\beta_{h} \Gamma_{h} \sim 1\right)$ in the outer parts of the star (see also Section 3.4.1). This is due to our lower-than-standard choice of $2 M_{\odot}$ for the progenitor mass. In this case, the breakout time is $t_{b, *} \simeq R_{*} / c$. Then, in general, 
the first condition on $t_{j}$ can be written

$$
t_{j} \geq \max \left(L_{j}^{-1 / 3} \rho_{*}^{1 / 3} R_{*}^{5 / 3} \theta_{0}^{4 / 3}, R_{*} / c\right)
$$

In order to derive the second condition, we first need an estimate of how long it takes for all of the matter emitted by the engine to flow into the cocoon. The last jet material is launched from the engine at $t_{j}$. Since the jet material below the head has velocity $\beta_{j} \approx 1$, the time for the tail end of the jet material to catch the head is $t_{\text {catch }} \approx t_{j} /\left(1-\beta_{h}\right)$. For a non-relativistic head, we get simply $t_{\text {catch }} \approx t_{j}$. For a relativistic head that is not in free expansion, we have $\Gamma_{h} \ll \Gamma_{0}$, so $t_{\text {catch }} \approx 2 \Gamma_{h}^{2} t_{j}$. Then, we can use Equations 1.5, 1.3, and 3.4 to write $t_{c a t c h}$ in terms of $\xi$, with the result:

$$
t_{\text {catch }}=\left\{\begin{array}{lc}
\xi^{1 / 5} \theta_{0}^{-4 / 5} t_{j}, & \theta_{0}^{4}<\xi<\theta_{0}^{2 / 3} \\
\xi^{1 / 2} \theta_{0}^{-1} t_{j}, & \theta_{0}^{2 / 3}<\xi<4 \theta_{0}^{2} \Gamma_{0}^{4}
\end{array} .\right.
$$

Finally, in the free expansion phase, $\Gamma_{h} \approx \Gamma_{0}$ and the time for all the material to catch up becomes long. We can write $\Gamma_{h}^{2} \approx \Gamma_{0}^{2}(1-\varepsilon)$, where $\varepsilon$ is a small parameter. Then $t_{\text {catch }} \approx 2 \Gamma_{0}^{2} t_{j} / \varepsilon$. Sari \& Piran (1995) studied the dynamics in this coasting limit; using their results, we find $\varepsilon \simeq \Gamma_{0}^{2} / f$, where $f \sim L_{j} /\left(\Gamma_{0}^{2} \theta_{0}^{2} A c^{3}\right)$ is the ratio of comoving rest-mass densities in the jet and ambient medium. Finally, substituting this back into the expression for $t_{c a t c h}$, we get $t_{c a t c h} \simeq \xi^{1 / 2} \theta_{0}^{-1} t_{j}$, the same as in the other uncollimated regimes.

The jet will be choked if $t_{\text {catch }}$ is less than the time to break out of the extended envelope, $t_{b, e x t}$. For typical parameters, the jet is relativistic in the envelope, and therefore $t_{b, e x t} \simeq R_{e x t} / c$. If $L_{j}$ is small or $A$ is large, however, the head could become non-relativistic, in which case $t_{b, e x t} \simeq L_{j}^{-1 / 3} A^{1 / 3} R_{e x t} \theta_{0}^{4 / 3}=\xi^{-1 / 3} \theta_{0}^{4 / 3} R_{e x t} / c$ instead. We define $t_{e s c}$ as the critical jet duration for which $t_{c a t c h}=t_{b, e x t}$, i.e. the final jet 
material catches the head just as it breaks out of the envelope. Combining results from the previous two paragraphs, we arrive at a piecewise expression for $t_{\text {esc }}$ :

$$
t_{e s c}=\left\{\begin{array}{lr}
\xi^{-1 / 3} \theta_{0}^{4 / 3} R_{e x t} / c, & \xi<\theta_{0}^{4} \\
\xi^{-1 / 5} \theta_{0}^{4 / 5} R_{e x t} / c, & \theta_{0}^{4}<\xi<\theta_{0}^{2 / 3} \\
\xi^{-1 / 2} \theta_{0} R_{e x t} / c, & \theta_{0}^{2 / 3}<\xi
\end{array} .\right.
$$

The jet is choked if $t_{j}<t_{e s c}$, and breaks out succesfully if $t_{j}>t_{e s c}$.

In Fig. 3.2, we plot $t_{b, *}, t_{b, e x t}$, and $t_{e s c}$ as functions of $\xi$. We also show where the numerical models considered here and the analytical models of Irwin \& Chevalier (2016) and Nakar (2015) lie in the $t_{j}-\xi$ parameter space. Because one does not expect a marked change in the dynamics if the jet is choked near the edge of the envelope, we also plot $0.2 t_{j}$; models that fall below this line are choked deep within the envelope. The black lines in the figure divide the parameter space into five distinct regions. In region 1, the jet escapes the envelope while the engine is still active. In region 2, the engine shuts off while the jet head is in the envelope, but the jet still escapes the envelope before all of the energy flows into the cocoon. In other words, the jet head does not become aware that the engine has shut off until after it crosses into the CSM, and as a result the dynamics in the envelope are the same as if the engine had stayed on. The situation in region 3 is the same as in region 2, except that in this case the jet always escapes regardless of its duration. In this regime the jet cannot be choked, as long as it successfully makes it out of the star. Region 4 indicates the part of parameter space where the jet is choked by the envelope. We further divide this area into region $4 \mathrm{a}$ and $4 \mathrm{~b}$, to separate jets which stall after they have already crossed a considerable fraction of the envelope (4a) from those which are choked deep in the envelope, before they reach $0.2 R_{e x t}(4 \mathrm{~b})$. Finally, in region 5 the jet duration 
is not sufficient to penetrate the host star. This is the regime that has been explored in past numerical studies of choked jets, as described in Section 3.1.

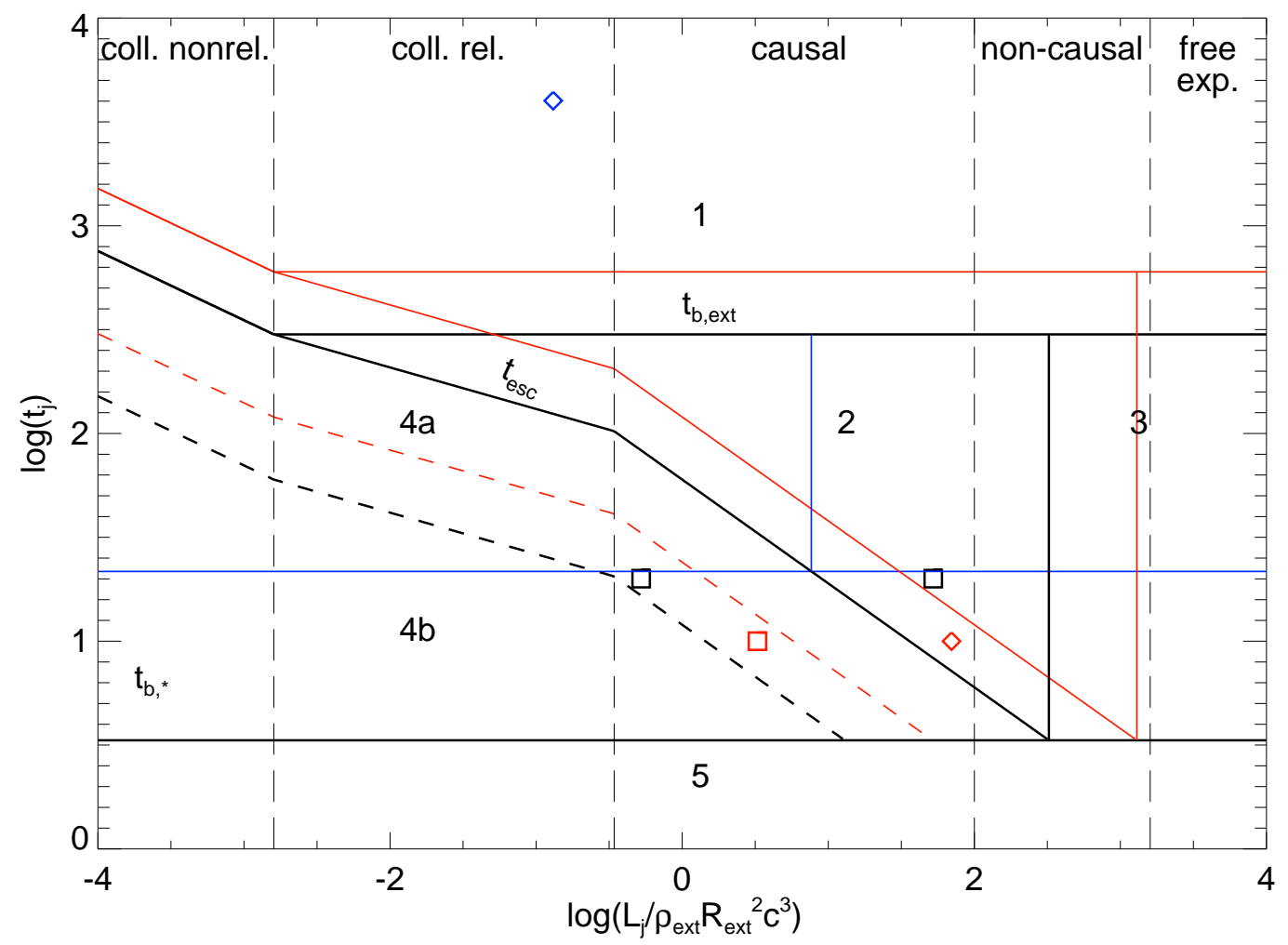

Fig. 3.2.- Characteristic timescales $t_{b, *}, t_{b, e x t}$, and $t_{e s c}$ versus the dynamical parameter $\xi$. The vertical dashed lines separate the plot into the five regimes discussed in Section 1.2, for $\theta_{0}=2$. Our models t20M4R9a2 and t20M400R9a2 are shown as black squares, and model t10M12R18a2 is plotted as a red square. Also, for reference, we show the analytical models of Irwin \& Chevalier (2016) (blue diamond) and Nakar (2015) (red diamond). The blue line shows how decreasing $L_{j}$ to the value suggested by Irwin \& Chevalier (2016) affects $t_{b, *}$, and the red line shows how increasing $R_{e x t}$ to the value suggested by Nakar (2015) affects $t_{\text {esc }}$ and $t_{b, e x t}$. The numbered sections indicate different behaviors, as discussed in the text.

The effect of varying each model parameter (holding other parameters fixed) is as follows:

- Increasing (decreasing) $M_{*}$ (or $R_{*}$ ) moves the $t_{b, *}$ line up (down). 
- Increasing (decreasing) $t_{j}$ moves a model up (down) in the space.

- Increasing (decreasing) $L_{j}$ moves a model up (down) in the space, and also moves the $t_{b, *}$ line down (up).

- Increasing (decreasing) $\theta_{0}$ moves the vertical dashed lines farther apart (closer together $)^{2}$.

- Increasing (decreasing) $\Gamma_{0}$ moves the rightmost vertical line to the right (left)

- Increasing (decreasing) $M_{\text {ext }}$ moves a model left (right) in the space.

- Increasing (decreasing) $R_{\text {ext }}$ moves a model right (left) in the space, and also moves the $t_{b, e x t}$ and $t_{e s c}$ lines up (down).

Changing $\alpha_{\text {ext }}$ significantly complicates matters, because when $\alpha_{\text {ext }} \neq 2, \xi$ is no longer constant but becomes a function of $t$.

The above considerations give us a framework in which to interpret the numerical results. We see that our models models t20M4R9a2 and t20M4R9a0 are not expected to undergo choking. These models should continue to behave like a jet while in the envelope. On the other hand, models t20M400R9a2 and t10M12R18a2 are deep within the choked-jet regime (the red square should be compared with the red lines in Fig. 3.2). Therefore, we might expect the dynamics in these models to differ from the jet case, possibly leading to a quasi-spherical explosion that could produce a shock breakout event as described by Nakar (2015).

\footnotetext{
${ }^{2}$ This is the reason for our choice to use $\xi$ rather than $\tilde{L}$. If $\tilde{L}$ were used instead, changing $\theta_{0}$ would also shift models left or right in the parameter space, and the amount of the shift would differ depending on whether or not the jet was collimated. Factoring out the $\theta_{0}$ dependence removes this complication and makes the results easier to intuit.
} 


\subsection{Results}

\subsubsection{Model t20M4R9a2: "Standard" parameters}

The four parameters varied in our models are $t_{j}, M_{e x t}, R_{\text {ext }}$, and $\alpha_{\text {ext }}$. Our naming convention lists the parameters with $t_{j}$ in units of s, $M_{e x t}$ in units of $10^{-3} M_{\odot}$, and $R_{\text {ext }}$ in units of $10^{12} \mathrm{~cm}$. So, model t20M4R9a2 has $t_{j}=20 \mathrm{~s}, M_{\text {ext }}=4 \times 10^{-3} M_{\odot}$, $R_{e x t}=9 \times 10^{12} \mathrm{~cm}$, and $\alpha_{e x t}=2$.

For this model, $M_{\text {ext }}$ and $R_{\text {ext }}$ are chosen based on the results of Section 2.4.3. The choice $t_{j}=20 \mathrm{~s}$ ensures that the jet comfortably breaks out of the star, while keeping the total jet energy $E_{j}=L_{j} t_{j}=2 \times 10^{51}$ ergs in line with the suggestion of Nakar (2015). We take $\alpha_{e x t}=2$ for most models; this is a typical value for stellar winds, and also enables us to invoke the machinery of Section 3.3.

We begin by examining the behavior of the jet within the progenitor star and comparing it to previous studies. Fig. 3.3 shows the jet just prior to breaking out of the star in our model, as compared to models from Morsony et al. (2007) and Mizuta et al. (2006). Morsony et al. (2007) used the same stellar density profile and stellar radius that we adopt, but a higher jet luminosity $\left(5 \times 10^{50} \mathrm{erg} \mathrm{s}^{-1}\right)$ and a larger stellar mass $\left(15 M_{\odot}\right)$. While the overall structure of the system (a narrow jet spine encased in a region of jet back flow, surrounded by a cocoon of shocked stellar gas) is the same, we find that the cocoon is somewhat narrower at the time of breakout compared to their model. The discrepancy can be attributed to two factors. First, even with their higher luminosity, the larger stellar mass results in a wider and slower flow. Second, and more importantly, they inject the jet in an uncollimated state, instead of injecting it parallel to the axis as we do here. This results in a jet that is slower initially, until the cocoon builds enough pressure to collimate the jet; it also 
explains why the Morsony et al. (2007) model is wider in particular near the base of the jet.

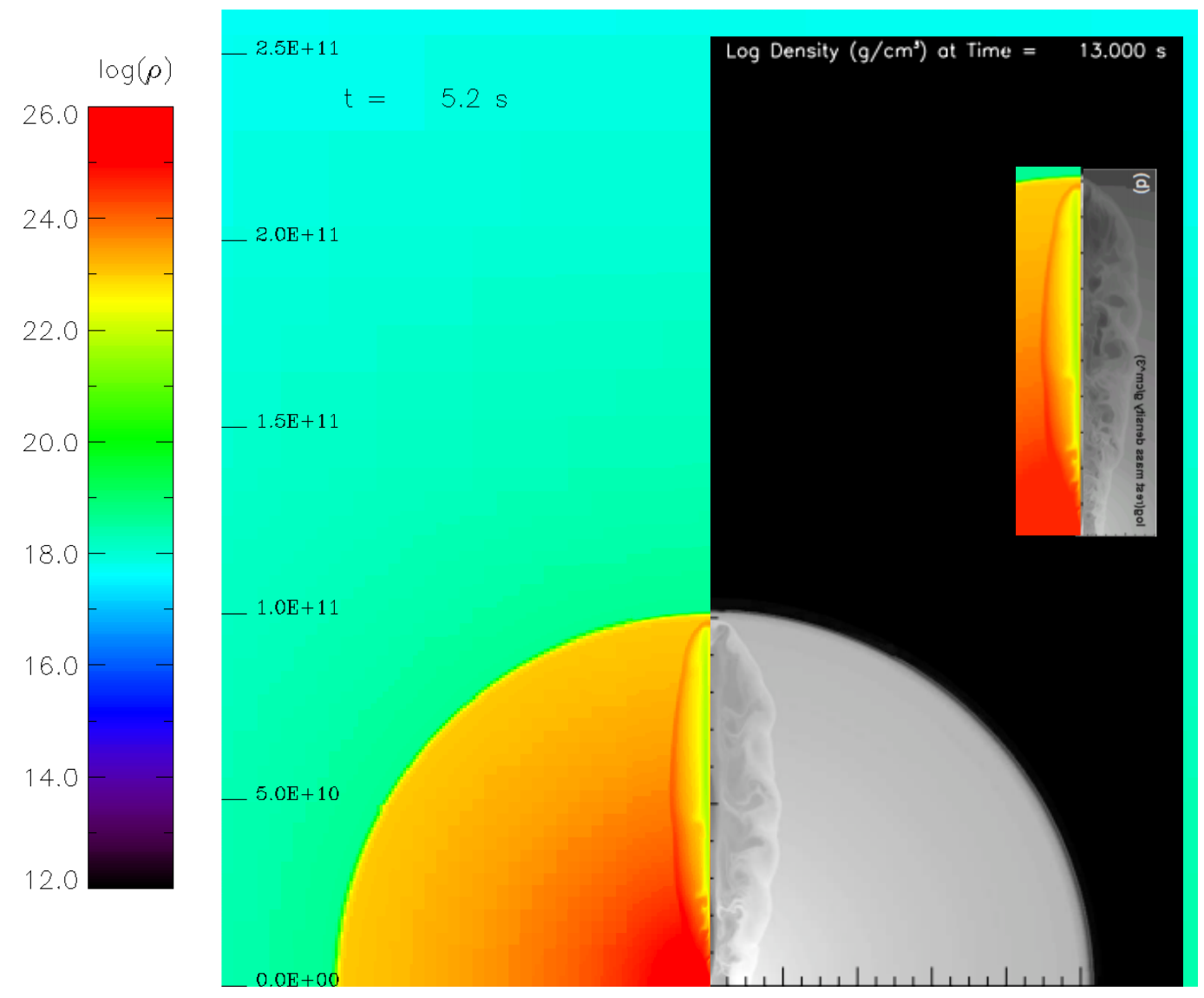

Fig. 3.3.- Main plot: Snapshot of model t20M4R9a2 just prior to breaking out of the star, comparing our model (color) to that of Morsony et al. (2007) (grayscale). Inset: Comparison between our model (color) and Mizuta et al. (2006) (grayscale). The Mizuta et al. (2006) model has been rescaled for the sake of comparison. The color bar gives the density in units of $m_{p}$ per $\mathrm{cm}^{3}$, where $m_{p}$ is the proton mass, and the ticks on the left give $z$ in $\mathrm{cm}$. See text for discussion.

Our results can also be compared to those of Mizuta et al. (2006). Like us, they inject the jet parallel to the z-axis. However, like Morsony et al. (2007), they used a higher luminosity $\left(10^{51} \mathrm{erg} \mathrm{s}^{-1}\right)$ and a higher stellar mass $\left(\sim 10 M_{\odot}\right)$. They also adopted a smaller stellar radius $\left(3.7 \times 10^{10} \mathrm{~cm}\right)$ and a different, steeper stellar density profile. Despite these differences, the overall morphology of the jet at breakout in 
our model bears a strong resemblance to the models found in Mizuta et al. (2006). This suggests that the method of jet injection has a substantial role in shaping the breakout conditions, a phenomenon that has also been discussed by Mizuta \& Aloy (2009). As mentioned in Section 3.2.2, parallel jet injection better reflects the physical conditions, because the jet is produced at a radius much smaller than the position of the simulation boundary.

We can compare our numerical result to analytical predictions, as well. In our models, the value of the dynamical parameter $\tilde{L}$ near the outer edge of the star is $\tilde{L} \simeq 1.1$. In this limit, neither the non-relativistic nor the relativistic solutions strictly apply, making a direct comparison difficult. However, we do expect that $\beta_{h} \Gamma_{h}$ will be roughly unity in this case. Our breakout time of $5.2 \mathrm{~s}$ suggests an average value of $\beta_{h}=0.64$ in the star, which corresponds to $\beta_{h} \Gamma_{h} \simeq 0.8$, confirming this expectation. When $\tilde{L} \sim 1$, the Bromberg et al. (2011) model also predicts that the width of the cocoon should be $\sim R_{*} \theta_{0} \sim 2 \times 10^{10} \mathrm{~cm}$. The width in the simulation, $1 \times 10^{10} \mathrm{~cm}$, is somewhat smaller than the simple analytical estimate, but this is within reason considering the crudeness of the analytical model. Mizuta \& Ioka (2013) also commonly found discrepancies of a factor of a few when comparing to the Bromberg et al. (2011) solution, so we do not view this result as unreasonable.

As each of the four models considered here use the same parameters for the host star and the jet ${ }^{3}$, the dynamics are identical in all models until the jet crosses into the envelope.

Next, we look at the jet-cocoon system as it traverses the envelope. After the jet breaches the stellar surface, the cocoon of shocked stellar gas, which is overpressured compared to the envelope, rapidly expands. The jet is no longer confined, and un-

\footnotetext{
${ }^{3} t_{j}$ is varied, but as it is longer than the stellar breakout time in all models, this does not affect dynamics within the star
} 
dergoes a brief period of free expansion, until it is eventually recollimated by a new cocoon of shock-heated envelope material. Then, the engine turns off. A low pressure region is left behind as the remaining jet material flows into the cocoon. This low pressure region is gradually crushed by the higher pressure cocoon as the outflow expands.

By the time the jet breaks out, we find that the cocoon is marginally causally connected, with a mostly uniform pressure throughout. Fig. 3.4 shows the state of the system just before breaking out of the envelope. This agrees with our expectations, as this model is close to the border between the causal and non-causal regimes (see Fig. 3.2. The Lorentz factor of the jet head is $\Gamma \simeq 4$ at breakout, which is also close to the analytical estimate. Additionally, the envelope breakout time is very nearly $R_{\text {ext }} / c$. However, the Bromberg et al. (2011) model predicts a much wider outflow than what we observe. The analytical model predicts $\beta_{c} \sim 1$, and therefore a nearunity aspect ratio $r_{c} / z_{h} \sim \beta_{c} / \beta_{h}$, where $z_{h}$ is the $z$-position of the jet. We find an aspect ratio of 0.3 instead. The cocoon pressure is also a factor of several lower than the analytical prediction.

Both of these features are related to the behavior of the jet material below the head. As long as the engine is active, the jet flows freely outward into a cone around the axis. However, once the jet shuts off and a significant fraction of the remaining jet material has flown into the head, the cocoon begins to encroach on this cone of space. As a result, the remaining jet material is somewhat more confined, and has a somewhat lower Lorentz factor (and specific internal energy) than would be the case had the engine remained on. Because the material is slower, it takes longer to catch up to the head, and energy flows into the cocoon at a reduced rate. This leads to a lower pressure, and therefore a slower lateral expansion, than in the analytical 
picture.

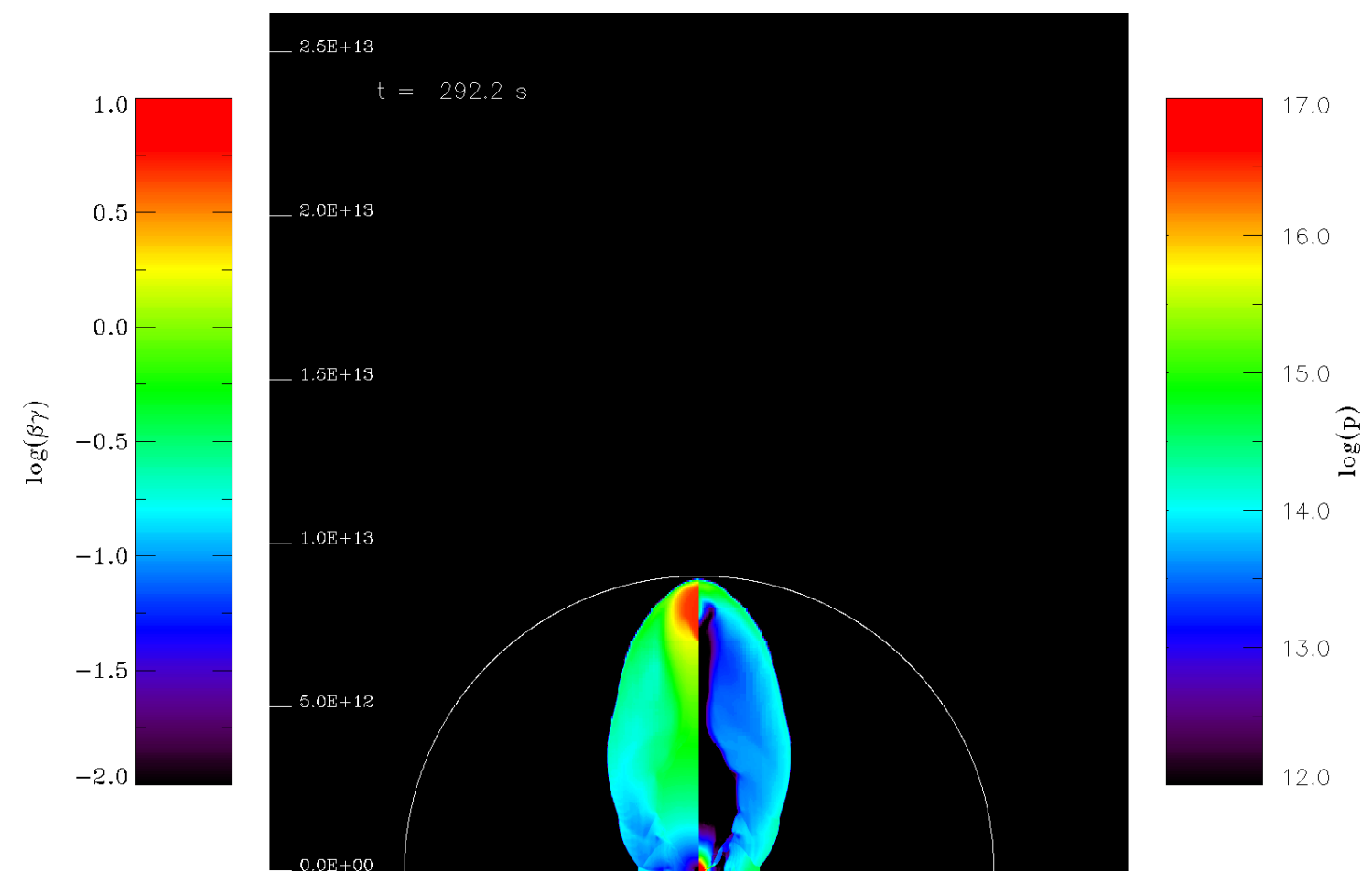

Fig. 3.4. $-\beta \Gamma$ (left) and pressure (right) for model t20M4R9a2 at $t=292 \mathrm{~s}$. Here and in subsequent plots, the pressure is expressed in units of $m_{p} c^{2}$ per $\mathrm{cm}^{3}$, and the edge of the envelope is indicated by the white line.

Lastly, we briefly discuss the behavior of the outflow in the low-density CSM. Fig. 3.5 shows the outflow $300 \mathrm{~s}$ after breakout. By this time, little remains of the jet; the dynamics are set by the cocoon. After breakout, the cocoon undergoes rapid, quasi-spherical adiabatic expansion. Because the cocoon pressure $p_{c} \gg \rho_{C S M} c^{2}$, where $\rho_{C S M}$ is the ambient CSM density, this expansion is relativistic. The sphericity of the outflow beyond the envelope has important observational implications: it means that even if the outflow is aspherical at breakout and the high-energy emission is beamed, off-axis observers can still detect a choked jet breakout via its radio emission. This is a crucial difference between GRB models with and without a dense envelope. Without the envelope, the jet goes into free expansion after breakout; most of the 
jet energy gets trapped in the jet head, and it can take a long time for the outflow to sphericize (see Section 2.4.5 for further discussion). In the dense envelope case, however, since most of the jet energy has already entered the cocoon prior to breakout, the transition to a spherical outflow within the CSM is much faster, and off-axis observers see radio emission from early times.

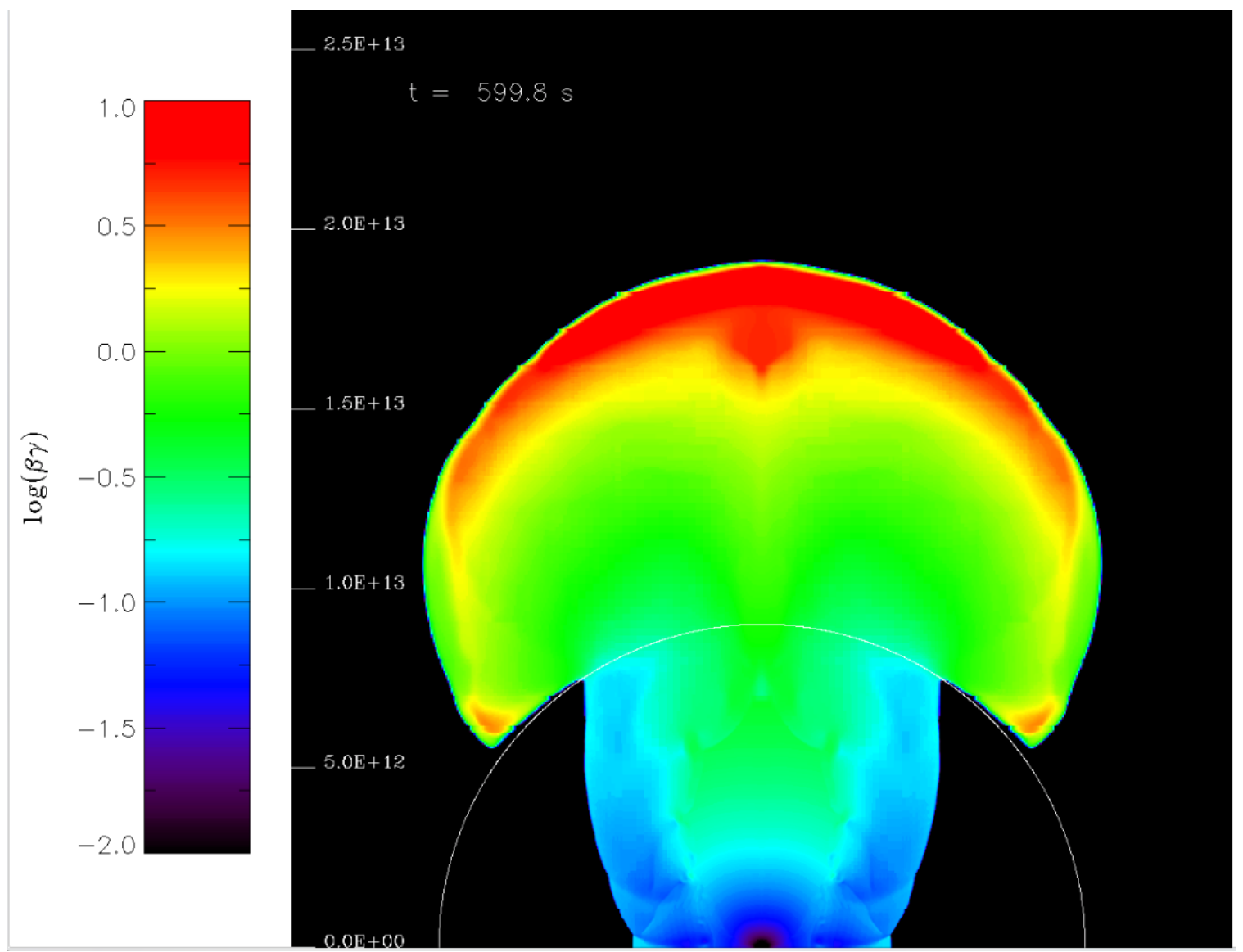

Fig. 3.5.- $\beta \Gamma$ for model t20M4R9a2 at $t=600 \mathrm{~s} \approx 2 t_{b, \text { ext }}$. 


\subsubsection{Model t20M4R90a0: The effect of the envelope density profile}

In this model, we explore the effect of changing the envelope density profile index from $\alpha_{e x t}=2$ to $\alpha_{e x t}=0$. The other parameters are kept the same as in the previous section. A snapshot of this model right before envelope breakout is shown in Fig. 3.6. Immediately it can be seen that the overall morphology is not greatly affected by the change in $\alpha_{\text {ext }}$. This is because the breakout dynamics are set mostly by the conditions in the outer layers of the envelope, and the density there is not so different from the $\rho \propto R^{-2}$ case.

However, there are some subtle differences. In particular, because the density close to the star is lower in the $\alpha_{\text {ext }}=0$ model, the period of free expansion just after the jet breaks out of the star is longer, and it takes more time for the envelope to recollimate the cocoon. This results in an outflow that is wider towards the equator, and a larger low-pressure region compared to the wind-like density profile. Even so, by the time the breakout occurs, the solution tends towards the same marginally causally connected state as in model t20M4R9a2. We therefore conclude that the mass distribution within the envelope has only a minimal effect on the physics at breakout.

\subsubsection{Model t20M400R9a2: A high-mass envelope}

Next, we consider a model with the same jet properties and envelope radius, but a much higher envelope mass. We make this choice so that the dynamics will enter the strongly choked regime, without having to increase the envelope radius (which is constrained by the total size of the grid, as discussed in the next section). We note that the envelope mass in this model, $0.4 M_{\odot}$, is close to the maximum mass allowed 


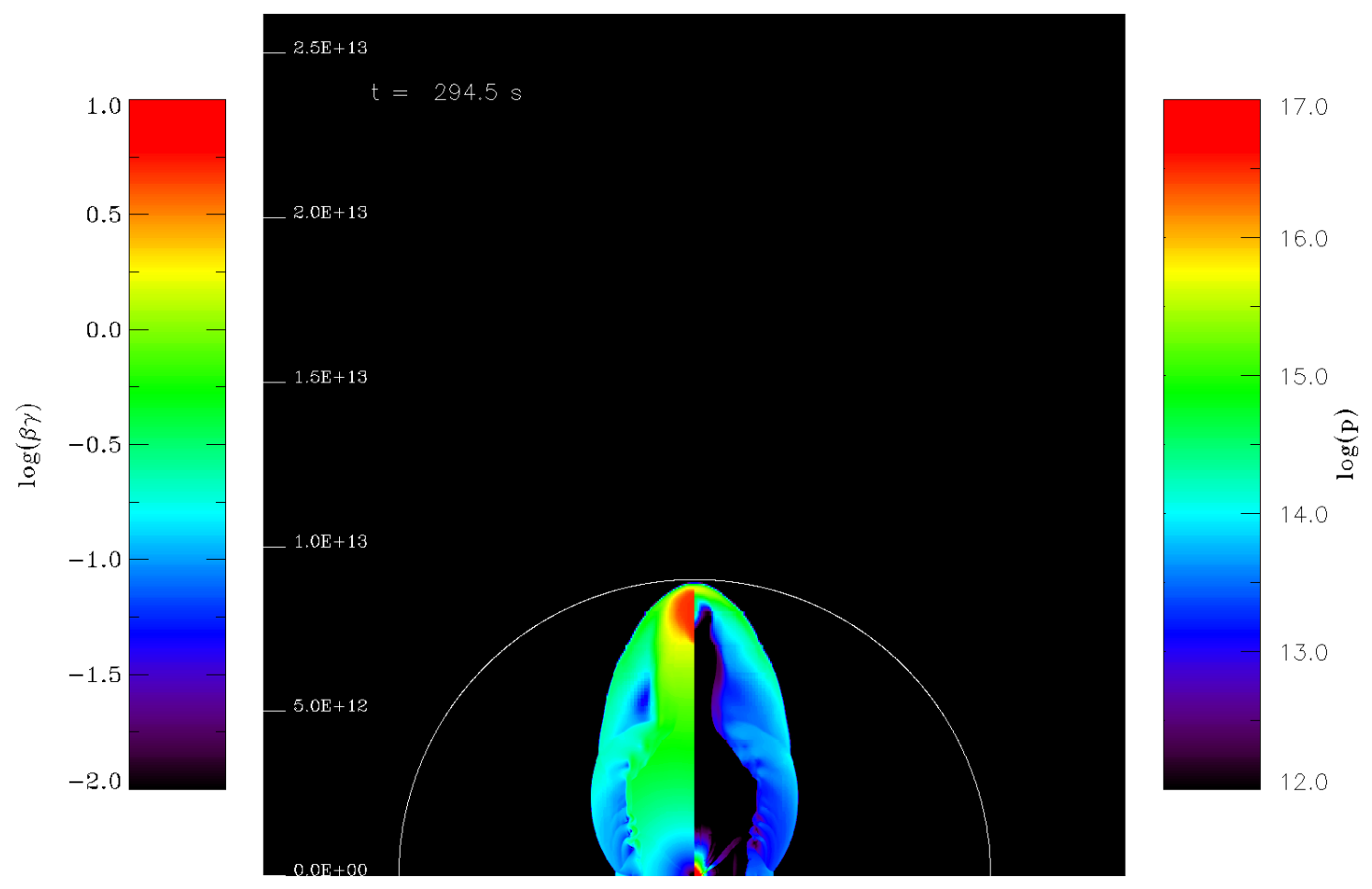

Fig. 3.6. $-\beta \Gamma$ (left) and pressure (right) for model t20M4R9a0 at $t=295 \mathrm{~s}$.

by the cooling envelope emission model, which requires $M_{\text {ext }} \ll M_{*}$. The value of $t_{j} / t_{\text {esc }}$ is about 0.2 in this model (see Fig. 3.2). We therefore expect that the jet will become choked, possibly leading to a quasi-spherical explosion as is seen when the jet is choked by the host star (Lazzati et al. 2012).

However, the results differ strikingly from this prediction, as we see in Fig. 3.7. The high envelope density leads to a large degree of jet confinement, which persists until breakout. Even though essentially all of the jet energy flows into the cocoon, there is not sufficient time for this narrow cocoon to become spherical before the head reaches the envelope edge. As the sideways expansion of the jet is much slower than the sound speed in the relativistic cocoon material, $\sim c / \sqrt{3}$, complete pressure equilibrium is reached in the cocoon.

The basic reason for the discrepancy between prediction and result is that the 
model parameters place the jet near the collimated regime. In this limit, the head of the jet is relativistic, while the lateral speed of the cocoon is non-relativistic. After all of the energy has flowed into the cocoon, the outflow begins to decelerate in both the forward and sideways directions. However, since the head is initially relativistic, its velocity remains close to $c$ even though it decelerates. As a result, the cocoon actually stretches itself out as it propagates at first. The head speed eventually drops below $c$, and continues to decrease until it is roughly equal to $\beta_{c}$; at this point the outflow can at last be considered spherical. Calculating the timescale for this transitional process to complete is not straightforward, because the system is not in a steady state. However, our results suggest that it can take a time at least comparable to the envelope crossing time, so that the cocoon does not sphericize until it has expanded to several times its original size. This seems consistent with the numerical results discussed in Section 2.4.5, which share the generic feature that the relativistic outflow takes much longer to transition from beamed to spherical than analytical estimates would suggest. This marks another departure from the case where the jet is choked inside the star. Because the head is already non-relativistic at the time of choking in that scenario, the transition to spherical flow is more rapid.

To roughly determine the shape of the outflow in this transitional regime, we consider the lateral expansion speed of a cocoon of constant energy $E_{c} \sim L_{j} t_{j}$. The pressure in this case can be estimated by $p_{c} \sim E_{c} /\left(\beta_{c}^{2} \beta_{h} c^{3} t^{3}\right)$. Assuming the cocoon speed is non-relativistic (we verify that this is self-consistent below), it is set by balancing the cocoon pressure against the envelope ram pressure, $\beta_{c} \sim\left(p_{c} / \rho_{\text {ext }} c^{2}\right)^{1 / 2}$. Eliminating $p_{c}$ from the equations and further approximating $\beta_{h} \approx 1$, we solve for $\beta_{c}$ to find

$$
\beta_{c} \sim\left(\frac{E_{c}}{\rho_{e x t} c^{5} t^{3}}\right)^{1 / 4} \sim\left(\frac{L_{j} t_{j}}{A c^{3} t}\right)^{1 / 4} \sim \xi^{1 / 4}\left(\frac{t_{j}}{t}\right)^{1 / 4}
$$


In the second step we have taken an $R^{-2}$ density profile. For the model described here, $\xi=0.5$, so $\beta_{c} \ll 1$ for all $t \gg t_{j}$, confirming our assumption of a Newtonian cocoon speed. An alternate way to derive this result is to consider the cocoon as a non-relativistic, cylindrical blast wave driven by energy per unit length $\lambda$ along the axis. Then one obtains the radius $r_{c} \sim\left(\lambda t^{2} / \rho_{\text {ext }}\right)^{1 / 4}$ by dimensional analysis, and the velocity is $\beta_{c} \sim\left(\lambda / \rho_{\text {ext }} t^{2} c^{4}\right)^{1 / 4}$. Equation (3.8) is reproduced when $\lambda \sim E_{c} /(c t)$. The result also agrees with the model of Bromberg et al. (2011), if their approximation for the energy in the cocoon, $E_{c} \sim L_{j} t / \Gamma_{h}^{2}$, is replaced by $E_{c} \sim L_{j} t_{j}$.

Thus, unless the jet choking occurs very deep within the envelope, a jet that is relativistic and collimated (or nearly collimated) does not break out spherically, but rather is elongated along the propagation direction. Numerous factors, however, make it difficult to construct a model that is very deep in the choked regime, as we will discuss in Section 3.5. Therefore, the best way to end up with an explosion that is spherical when it breaks out of the envelope is for the outflow to already be roughly spherical when the jet is choked. This generally only occurs when $\xi$ is large enough that both $\beta_{c} \sim 1$ and $\beta_{h} \sim 1$ hold. When this is not the case, the elongated outflow may produce an aspherical shock breakout as described by Matzner et al. (2013), and may look different than from the picture suggested by Nakar \& Sari (2012) and Nakar (2015), who assumed a spherical shock breakout model.

\subsubsection{Model t10M12R18a2: A short-lived jet in a large en- velope}

Considering the results of the previous section, it is desirable to devise a model for which choking occurs deep within the envelope, but the conditions at $t_{\text {catch }}$ are such that the outflow is already quasi-spherical. The best way to do this is to increase the 


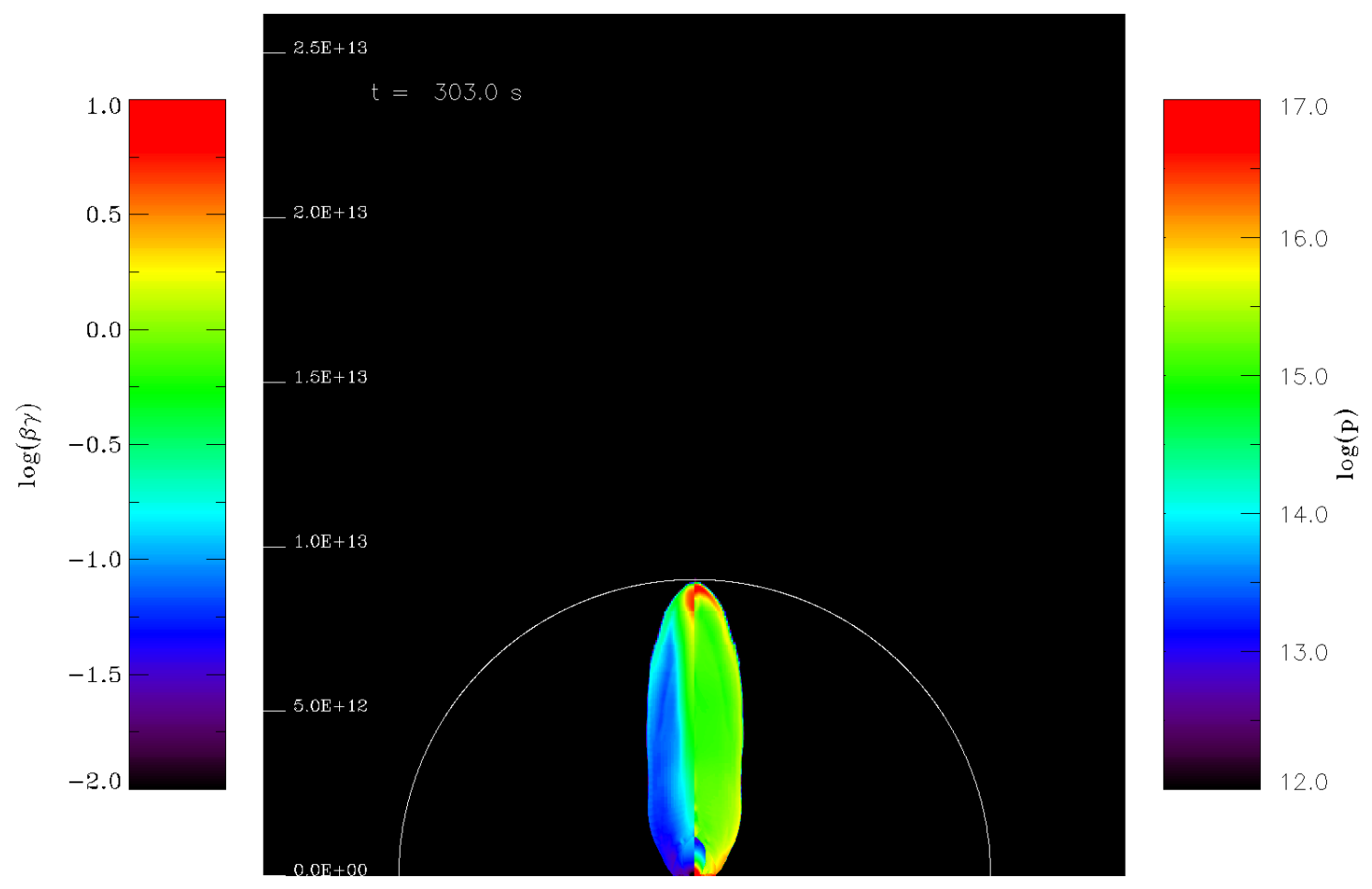

Fig. 3.7. $-\beta \Gamma$ (left) and pressure (right) for model t20M400R9a2 at $t=303 \mathrm{~s}$.

size of the envelope. However, because $t_{j} / t_{e s c} \propto R_{e x t}^{1 / 2}$, a large increase in $R_{\text {ext }}$ compared to model t20M4R9a2 is required to get a small value of $t_{j} / t_{\text {esc }}$. For practical reasons, greatly increasing $R_{e x t}$ is not ideal, because the computational grid would need to be made larger as well. Recalling that the highest resolution in the problem is set by the jet width at the injection site (which has no dependence on the envelope properties), we see that increasing the size of the grid necessarily increases the dynamic range of the problem, making simulations of very large envelopes expensive. Furthermore, optical observations generally constrain $R_{e x t}$ to be in the range of a few $10^{12} \mathrm{~cm}$ to a few $10^{13} \mathrm{~cm}$ (Nakar 2015; Drout et al. 2016; Irwin \& Chevalier 2016; Taddia et al. 2016). Another option is to decrease $t_{j}$ directly, but this runs into a limit because it must remain somewhat longer than $t_{b, *}$. Therefore, we compromise by increasing $R_{\text {ext }}$ by a factor of 2 , reducing $t_{j}$ by a factor of two, and then increasing the 
envelope mass to $0.128 M_{\odot}$. This results in about the same value of $t_{j} / t_{e s c}$ as in model t20M400R9a2, but a value of $\xi=3.5$ that places the jet firmly in the uncollimated regime.

We show the results of this simulation in Fig. 3.8. Compared to the previous model, it is indeed wider at the time of breakout, as expected for the larger value of $\xi$. We again find a uniform, albeit lower, pressure in the cocoon. Some effects indicative of the transition to spherical flow are apparent; in particular, the center of the cocoon begins to bulge out as it propagates in the outer envelope. However, the flow is still far from spherical when it breaks out.

The analytical solution of Bromberg et al. (2011) predicts an aspect ratio of $\sim 0.8$ before the jet is choked, so why is the numerical result still highly aspherical at breakout? The answer comes back to the same effect discussed in Section 3.4.1: the cocoon pressure partially confines the straggling jet material, so that energy flows into the cocoon at a slower rate, and the cocoon pressure and cocoon speed are lower after the engine shuts off. Consequently, the cocoon aspect ratio is reduced by a factor of a few; it is only 0.3 when the jet is choked, and decreases to about 0.2 before breakout, dropping roughly as $t^{-1 / 4}$ consistent with equation (3.8).

\subsection{Discussion}

Based on the results of the four models discussed in Section 3.4, we conclude that it is rather difficult to produce a quasi-spherical explosion. As it turns out, it is hard to circumvent this difficulty due to physical limitations on the model parameters. As discussed in Section 3.4.3, $M_{\text {ext }}$ cannot be increased much (for constant $R_{e x t}$ ) without violating the requirement that $M_{\text {ext }} \ll M_{*}$ for the cooling envelope model. For the same reason, $R_{\text {ext }}$ cannot be increased much unless the envelope density is 


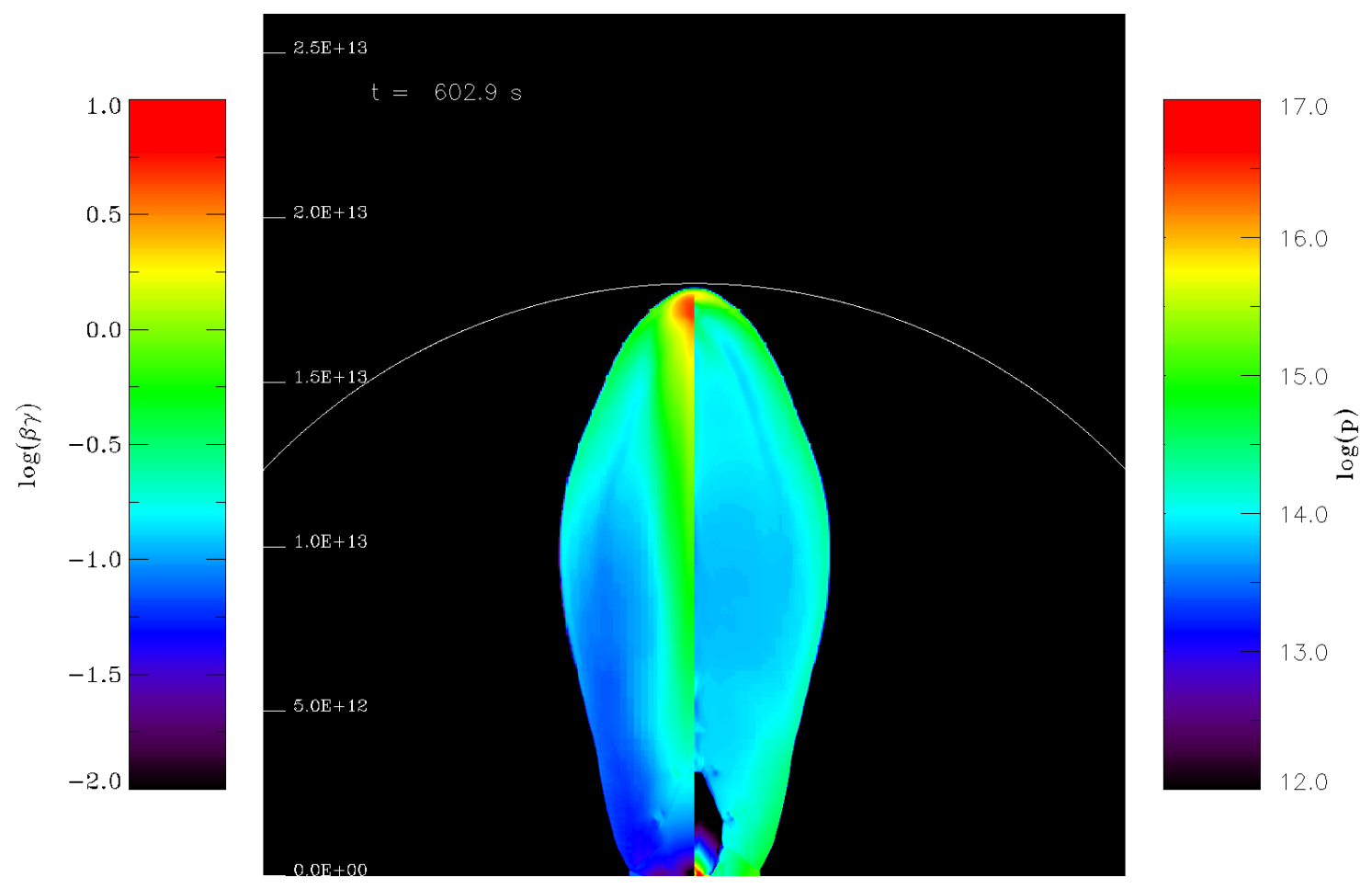

Fig. 3.8. $-\beta \Gamma$ (left) and pressure (right) for model t10M128R18a2 at $t=295 \mathrm{~s}$.

also decreased. $L_{j}$ cannot be increased much due to energetics; observed for standard GRBs are typically clustered near $E_{j} \sim 10^{51}$ ergs. Neither can it be decreased too far without $t_{b, *}$ becoming larger than $t_{j}$, and the same goes for decreasing $t_{j}$.

The best options to produce more favorable conditions for a spherical explosion (i.e., to decrease $t_{j} / t_{e s c}$ ), without running into any of the above limits, are to 1 ) increase $R_{\text {ext }}$ while lowering $\rho_{\text {ext }}$ to avoid a large increase in mass, or 2) decrease $L_{j}$ while increasing $t_{j}$. However, even in these cases there are drawbacks. For option 1, while there is no physical reason that very large envelopes cannot exist in nature, explaining the early optical peak requires more modest radii between $10^{12} \mathrm{~cm}$ and a few $10^{13} \mathrm{~cm}$, similar to what we have assumed here (Nakar 2015; Drout et al. 2016; Irwin \& Chevalier 2016; Taddia et al. 2016). For option 2, one problem is that decreasing $L_{j}$ moves the jet further towards the collimated regime, so that one encounters the 
situation described in Section 3.4.3. Furthermore, changing the jet parameters $L_{j}$ and $t_{j}$ to nonstandard values removes one of the attractive features of choked-jet shock breakout models for LLGRBs such as Nakar (2015), namely that an unusual jet is not required.

Our results have implications for the event rate of LLGRBs, as well. The Lorentz factor of the jet head is typically around $\sim 3-5$ when the outflow breaks out in our models, so that the breakout emission is somewhat beamed, into an opening angle $\theta_{b} \sim \Gamma_{h}^{-1} \sim 0.2-0.3$. While the beaming is less than for a jet that is not choked by the envelope, it is still significant. If this is the case the true event rate of choked-jet breakout events is increased compared to the prediction of a spherical model. As discussed in Section 2.5, this level of beaming results in a volumetric LLGRB rate that is close to the rate of broad lined Type Ic SNe (when the low end of the observed LLGRB event rate is used), suggesting that the progenitors of broad lined Type Ic SNe often are shrouded in dense envelopes. If the high end of the observed LLGRB rate is adopted, then beamed models result in a volumetric LLGRB rate that is higher than the rate of broad lined Type Ic SNe, which is not allowed. If both choked jets and successful jets produce beamed emission, as our results suggest, then a completely new interpretation of LLGRBs may be required if future observations observations constrain the LLGRB rate to be high.

Because of the difficulties in sphericizing the jet, it is also the case that most of the envelope is left intact after the crossing of the relativistic outflow. As a result, we expect interaction of the SN with the remaining envelope material to produce some or all of the prompt optical emission, as discussed in Section 2.4.3. We also saw that the outflow tends to be wide (but nonspherical) at breakout, and to sphericize shortly after breakout. The overall evolution of the outflow therefore closely resembles the "hybrid 
model" discussed in Chapter 2. In fact, it is hard to design a model that does not behave like the hybrid model, due to the parameter restrictions discussed above. Our numerical simulations therefore support the idea that the prompt emission of GRB 060218 could have been produced by a typical GRB jet launched into an extended envelope, with the X-ray emission coming from shock breakout of the cocoon as in Nakar (2015), the optical emission powered by interaction of the SN with the extended envelope as in Section 2.4.3, and the radio afterglow coming from the external shock of the post-breakout spherical outflow as in Barniol Duran et al. (2015). However, the possibility that GRB 060218 is powered by a long-duration, low-luminosity jet (as described in Chapter 2) cannot be excluded.

\subsection{Summary and Conclusions}

We conducted numerical simulations replicating the conditions of a GRB jet in the type of dense circumstellar envelope inferred from optical observations of doublepeaked SNe (Nakar \& Piro 2014). We studied the propagation of the jet through the progenitor star, the extended envelope, and the CSM, paying particular attention to the state of the jet-cocoon outflow just prior to emerging from the envelope. We also constructed an analytical model for jet choking in a $\rho \propto R^{-2}$ medium, and discussed the different physical regimes that are possible depending on combinations of the engine duration and the dimensionless parameter $\xi=L_{j} / \rho_{\text {ext }} R_{\text {ext }}^{2} c^{3}$.

We found similar results as previous authors (e.g., Morsony et al. 2007; Mizuta et al. 2006) for the propagation of the jet within the star. The few differences between our model and the Morsony et al. (2007) model can be traced to a different way of injecting the jet, and agree with the discussion in Mizuta \& Aloy (2009) and Mizuta \& Ioka (2013). 
We compared our numerical results to the analytical predictions of Bromberg et al. (2011) and to our analytical model presented in Section 3.3. While our results are in reasonable agreement with the analytical predictions of Bromberg et al. (2011) while the engine is active, they start to differ from the simple analytical picture after the engine shuts off, even before all of the jet material has caught up with the head at $t_{\text {catch }}$. The confinement of the tail end of jet material by the cocoon slows the rate of energy flow into the cocoon, and as a consequence the cocoon is narrower and its pressure is lower as compared to analytical models (Nakar 2015; Senno et al. 2016) that have assumed the dynamics stay the same until all of the jet material has flown into the cocoon. We also showed that if the initial condition at the time $t_{\text {catch }}$ is such that the jet head is relativistic while the cocoon is non-relativistic, then the transition to spherical takes longer than it otherwise would. Jets that are fully or partially collimated while propagating in the envelope satisfy this condition, and therefore have trouble sphericizing before emerging from the envelope, unless the envelope is significantly larger than is typical. The combination of these two effects (narrow-than-expected envelopes before $t_{c a t c h}$, and difficulty in sphericizing a narrow flow after $t_{\text {catch }}$ ) makes it difficult to obtain a quasi-spherical explosion under standard conditions, for most of the acceptable range of parameters. This has important implications for shock breakout models of LLGRBs (e.g., Nakar \& Sari 2012; Nakar 2015), which have generally assumed spherical symmetry.

We also found that, as long a significant fraction of the jet energy flows into the cocoon prior to breakout into the CSM, the outflow rapidly becomes spherical in the CSM, driven by the relativistic expansion of the overpressured cocoon. This is the case for the envelope parameters found by most authors (Nakar 2015; Drout et al. 2016; Irwin \& Chevalier 2016; Taddia et al. 2016), and is a key difference from the 
standard GRB model without an envelope, where as long as $t_{j}>t_{b, *}$, most of the energy ends up trapped in the jet head. This has ramifications for radio follow-up observations of double-peaked SNe, suggesting that a mildly relativistic synchrotron afterglow should generally be observable if a jet traversed the envelope, even for offaxis observers. The afterglow in this case would be similar to the one described by the shock breakout afterglow model of Barniol Duran et al. (2015).

Jet-envelope interaction is likely to play a major role in explaining the observed variety of $\gamma$-ray transients, and this work is only the first step towards a complete understanding of this process. Yet, some surprising differences from the basic analytical models already suggest that this is a fruitful avenue of research. Future numerical work will better sample the $\xi-t_{j}$ parameter space, giving us greater insight into the physics at play. Exploring the limit of long duration jets-both those with low luminosity as discussed in Chapter 2, and those with high luminosity as will be discussed in Chapter 4 - is one intriguing possibility.

\section{Acknowledgments}

This research was supported in part by NASA Grant NNX12AF90G. 
Chapter 4

Future Work: The Ultra-Long Gamma-Ray Burst Connection 


\begin{abstract}
We discuss a potential future research project to investigate the origin of a class of ultra-long gamma-ray bursts (ULGRBs) recently uncovered by Swift. Via a threepronged theoretical approach involving analytical calculations, spectral modeling, and numerical hydrodynamics simulations, we will test the hypothesis that the prompt $\mathrm{X}$-rays and $\gamma$-rays in ULGRBs arise from Comptonization of thermal photons in a moderately relativistic, dissipative jet that remains active for $\sim 10^{4} \mathrm{~s}$ or longer. As well, we will explore whether the early optical emission in these long bursts is compatible with interaction between an underlying supernova (SN) and an extended low-mass envelope surrounding the progenitor star. The results have important implications for the progenitors of this new class of $\gamma$-ray transient, and will furnish predictions for Swift and future $\gamma$-ray survey missions.
\end{abstract}

\title{
4.1 Introduction
}

As it turns out, the long-lived LLGRBs discussed in previous chapters are not the only peculiar subset of GRBs marked by unusually long duration. A never-before-seen "ultra-long" class of GRBs, with an even longer duration and a luminosity intermediate between GRBs and LLGRBs, has also recently been discovered by Swift (Levan et al. 2014). The extreme duration of these bursts poses a possible challenge to the standard "collapsar model" for long GRBs discussed in Section 1.1. As with LLGRBs, the implied rate per cosmic volume of this new variety of GRB transient is within a factor of $\sim$ a few of the rate of more typical GRBs; as a result, ULGRBs are just as critical to understanding GRB engine processes, high-mass stellar evolution, and the connection between GRBs and SNe. And, as with LLGRBs, these newly realized transient events remain poorly understood. 
In this penultimate chapter, we discuss a possible future research program aimed at answering key questions about the origin of ultra-long GRBs. Specifically, the questions we will seek to address are:

- What are the progenitor systems of ULGRBs? Are they similar to standard long GRBs (i.e., are ULGRBs powered by intrinsically long-lived central engines)? If so, what are the engine properties, and how do they compare to more typical bursts? Is a different type of central engine (e.g., a strongly magnetized neutron star, or "magnetar," as opposed to a black hole) required? If not, then what is their origin?

- What physical processes are responsible for the emission of ULGRBs at different wavelengths and epochs? How can these processes be unified under a comprehensive theoretical picture?

Settling these questions will be a further step towards a complete categorization of GRB transients, a task that will only grow in importance as our detection capabilities continue to increase.

\subsection{Background}

By and large, GRBs observed to date fall into one of two classes: short $(<2 \mathrm{~s})$ bursts with hard $\gamma$-ray spectra, and long $(>2 \mathrm{~s})$ bursts with comparatively soft spectra (Kouveliotou et al. 1993). Recently, however, Levan et al. (2014) have made the case for a separate population of ultra-long bursts. In particular, they argued that three long-duration events - GRB 101225A (Thöne et al. 2011), GRB 111209A (Gendre et al. 2013; Stratta et al. 2013), and GRB 121027A (Levan et al. 2014) - comprise a distinct subclass because they are clear outliers relative to other populations in the 
isotropic luminosity versus burst duration parameter space (see Fig. 4.1), and because of similarities in their unusual prompt emission. It is generally accepted that short and long GRBs have a different physical origin: short bursts are thought to originate from the merger of two compact objects (Nakar 2007), while the association of long bursts with SNe suggests that they arise from central engines formed by core-collapse in massive stars (Woosley \& Bloom 2006). Thus, it is natural to wonder whether ULGRBs may also derive from unique progenitor systems.

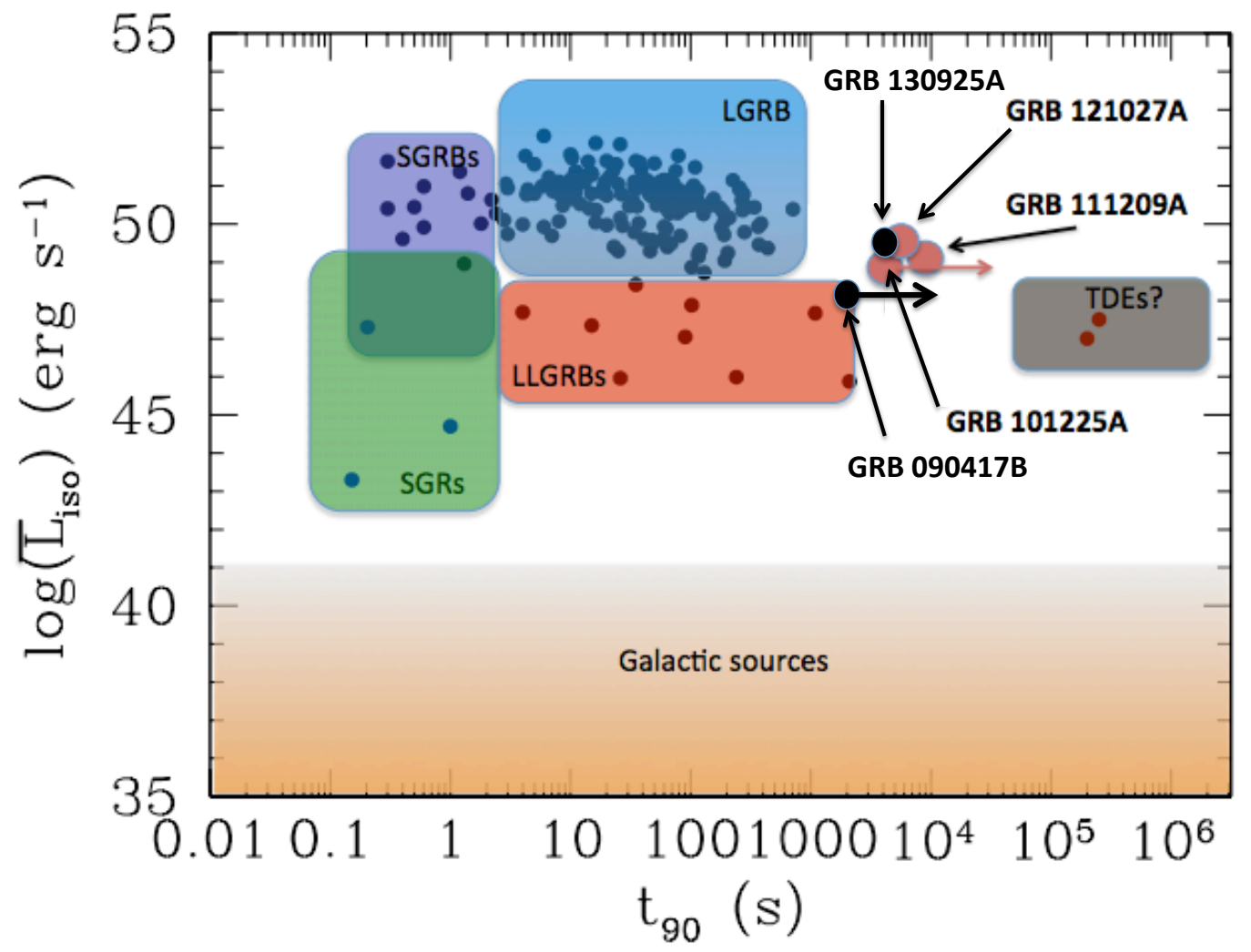

Fig. 4.1.- Isotropic luminosity versus duration for $\gamma$-ray transients, adopted from Levan et al. (2014) with GRB 090417B and GRB 130925A added as black dots. The separation of ULGRBs from other populations such as short GRBs (purple), long GRBs (blue), and tidal disruption events (gray) is apparent in this parameter space.

In addition to the bursts described by Levan et al. (2014), two other bursts that 
share broad similarities with the ULGRB class are GRB 090417B (Holland et al. 2010) and GRB 130925A (Evans et al. 2014). We summarize the multiwavelength properties of this ULGRB sample in Table 4.1. The unusual characteristics distinguishing this subclass from the usual GRB population are:

- A very long prompt emission timescale $\left(\sim 10^{4} \mathrm{~s}\right)$, and an average luminosity on the low end of the range for typical long GRBs, i.e. $L_{i s o} \sim 10^{49}-10^{50} \mathrm{erg} \mathrm{s}^{-1}$

- Highly variable prompt light curves exhibiting a complicated dipping and flaring behavior.

- A very steep decay of the prompt X-ray flux, followed by an X-ray afterglow. In GRB 111209A and GRB 121027A, this afterglow appears typical of long GRBs (Levan 2015). In GRB 090417B and GRB 130925A, however, the afterglow spectrum is unusually soft and the implied X-ray absorption column is high (Holland et al. 2010; Evans et al. 2014; see also Margutti et al. 2015). These cases have been modeled as echoes of the prompt emission from dust at tens of parsecs.

- Prompt optical/UV emission, peaking on a timescale of 0.1-1 days, that shows little correlation with the X-ray behavior (Thöne et al. 2011; Levan et al. 2014)

Soft, quasi-thermal X-ray emission is also observed at early times in several events (Thöne et al. 2011; Levan et al. 2014; Piro et al. 2014; Bellm et al. 2014). Radio data are available for GRB 111209A (Hancock et al. 2012) and GRB 130925A (Bannister et al. 2013; Horesh et al. 2015); the atypical radio spectrum in the latter case provides further evidence differentiating ULGRBs from normal long bursts.

In the case of GRB 101225A, there exists weak evidence for an associated SN, but the detection is not unambiguous (Levan et al. 2014). More recently, Greiner et al. 
(2015) claimed detection of a SN associated with GRB 111209A, SN 2011kl, based on an excess of emission with superluminous SN-like properties during the afterglow phase. Because the excess emission is bright, blue, and metal-poor, they suggest that the $\mathrm{SN}$ is powered by a magnetar - an important result that may hold clues as to the origin of ULGRBs.

Models that have been successful for other long-duration events seem to fall short when applied to ULGRBs. For example, some long duration transients detected by Swift, such as J1644+57 (Krolik \& Piran 2011) and J2058+05 (Cenko et al. 2012), appear to be powered by the tidal disruption of a star by a supermassive black hole. However, the relatively short timescale and high luminosity, as well as the lack of fallback emission with signature decay $t^{-5 / 3}$, make this explanation unlikely for ULGRBs (Levan et al. 2014; Evans et al. 2014). In addition, no accompanying SN is expected for a tidal disruption, contradicting the SN detection of Greiner et al. (2015). Relativistic shock breakout has also been proposed to explain some long-duration events (e.g., Nakar \& Sari 2012; Nakar 2015), but in this case it is difficult to explain the sharply varying light curve, and the simultaneous detection of nonthermal X-rays, thermal optical emission, and possibly thermal X-rays may also be problematic in the shock breakout view (see Irwin \& Chevalier 2016). Evans et al. (2014) suggest that the long burst duration in GRB 130925A is tied to a low-density circumstellar

\begin{tabular}{|l|c|c|c|l|l|l|l|}
\hline Object & $z$ & $\begin{array}{c}L_{\text {iso }} \\
\left(\mathrm{erg} \mathrm{s}^{-1}\right)\end{array}$ & $\begin{array}{c}\text { Duration } \\
(\mathrm{s})\end{array}$ & $\begin{array}{l}\text { Thermal } \\
\text { X-rays? }\end{array}$ & $\begin{array}{l}\text { Prompt } \\
\text { optical? }\end{array}$ & $\begin{array}{l}\text { X-ray } \\
\text { afterglow? }\end{array}$ & $\begin{array}{l}\text { Radio } \\
\text { detection? }\end{array}$ \\
\hline GRB 090417B & 0.345 & $\sim 10^{48}$ & $>2130$ & No & No & Dust echo & No \\
\hline GRB 101225A & 0.847 & $\sim 10^{49}$ & $\sim 2.2 \times 10^{4}$ & Maybe & Yes & No & No \\
\hline GRB 111209A & 0.677 & $\sim 10^{49}$ & $\sim 2.5 \times 10^{4}$ & Maybe & Yes & Typical & Yes \\
\hline GRB 121027A & 1.773 & $\sim 10^{50}$ & $\sim 1.4 \times 10^{4}$ & Unknown & Yes & Typical & No \\
\hline GRB 130925A & 0.348 & $\sim 10^{50}$ & $>5000$ & Maybe & No & Dust echo & Yes \\
\hline
\end{tabular}

Table 4.1: Summary of multiwavelength observations for ULGRB targets. 
medium (CSM), since in this case the reverse shock is slow and internal shocks have a longer time to extract energy. However, while this model works for GRB 130925A where the X-ray afterglow appears to be dominated by a dust echo, the more typical afterglows of GRB 111209A and GRB 121027A are not compatible with a low-density medium (Levan 2015). Last but not least, several authors have argued that ULGRBs are driven by the collapse of blue supergiants (BSGs) (e.g., Gendre et al. 2013; Levan et al. 2014; Nakauchi et al. 2013). However this, too, is inconsistent with 2011kl, because SNe originating from a BSG are expected to contain hydrogen, whereas $2011 \mathrm{kl}$ is hydrogen poor (Greiner et al. 2015).

A major goal of this research is to develop a unified theory for the ULGRB class that can be applied to all of its constituent events. Given the difficulties with the models above, the alternative that ULGRBs arise from intrinsically long-lived, possibly magnetar-driven central engines should be considered. This scenario is consistent with an associated magnetar-powered supernova such as $2011 \mathrm{kl}$. The possibility that long GRBs are powered by magnetars has been considered before (e.g., Usov 1992; Zhang \& Mészáros 2001; Metzger et al. 2011), but only recently has it been applied to ULGRBs (see, e.g. Fig. 1 in Metzger et al. 2015).

Furthermore, the several - albeit uncertain - claims of thermal X-ray emission, which are hard to interpret in other models, can be explained in a jet model if there is dissipation within the jet. As the suggested thermal X-ray components have timescales similar to the duration of nonthermal emission, it is natural to ask whether the prompt nonthermal radiation in ULGRBs could be produced via Comptonization of a thermal component. This interpretation has the advantage of linking the prompt emission mechanism of ULGRBs to recent work on typical GRBs, where this "photospheric model" for the prompt emission is also gaining traction (see, e.g. the dis- 
cussion in Sections 1.3, 2.4.1 and 2.4.7). Despite the advantages of the photospheric view, to date it has not been applied to the class of ULGRBs. The proposed research aims to tackle this problem; in the next section, I describe how.

\subsection{Methodology}

Our plan to investigate ULGRBs involves three phases. First, we will use analytical methods to constrain, to the extent possible, properties of the progenitor system, the circumstellar environment, and the central engine for each of the targets described in Section 2. Second, we will perform spectral fits of the high energy emission in the target ULGRBs, using a thermal component broadened by Comptonization to fit the data instead of the standard blackbody + power-law, cut-off power-law, or Band function fits. Finally, we will undertake more rigorous numerical simulations to ascertain the viability of photospheric models in producing ULGRB-like light curves.

For the first phase, our previous work on GRB 060218 can act as a starting point. Although its smooth light curve and exceedingly low luminosity situate it in a different subclass, GRB 060218 has a number of features in common with ULGRBs: its prompt emission is long-lived ( $3000 \mathrm{~s})$, displays a sharp decline, and contains a significant soft thermal component; it displays prompt optical emission with timescale $0.1-1$ day; and it was detected as a radio source at late times. In Chapter 2, we developed a comprehensive model for GRB 060218 and similar bursts; the same basic framework can potentially apply to the ULGRB class as well.

As an example, consider GRB 111209A. In this case, we have: 1) an estimate of the X-ray blackbody luminosity, if a blackbody + power-law fit for the prompt emission is assumed (Levan et al. 2014); 2) an estimate for the mass and energy of the associated SN 2011kl (Greiner et al. 2015); 3) an estimate of the radio flux at 
$5.5 \mathrm{GHz}, 9 \mathrm{GHz}$, and $18 \mathrm{GHz}$ at 5 days (Hancock et al. 2012); and, of course, 4) detailed light curves and spectra for the nonthermal X-rays and optical emission (Levan et al. 2014). Starting with the thermal emission, if this is modeled as emerging from the photosphere in a relativistic outflow, one can put a lower limit on the outflow Lorentz factor. Next, one can suppose that the early optical peak comes from interaction of the outer SN ejecta with an optically thick envelope. The timescale and luminosity of the peak, and the SN mass and energy, give an estimate for the envelope mass and radius (see, e.g. Nakar \& Piro 2014). The cooling envelope model for the early optical peak requires that the SN-envelope interaction be quasi-spherical; given the envelope mass and radius, this leads to an upper limit on the jet Lorentz factor and opening angle, because the jet must stay reasonably collimated in the envelope. In turn, an upper limit on the jet energy is implied, and if the jet energy is constrained, the radio observations (assuming a synchrotron model) can tell us something about the circumstellar density. This exercise can be repeated for the other bursts of interest. In the event that observations only give an upper limit, one can still derive upper or lower bounds on the physical parameters that give some idea of the underlying physics.

In the second phase, we will fit model spectra calculated assuming that Comptonization of thermal photons produced at the jet photosphere is the dominant radiation mechanism to the observed spectra of the ULGRB sample. Pe'er \& Waxman (2005) developed a code to calculate the spectrum of a relativistic plasma including jet dissipation, and Ahlgren et al. (2015) recently implemented the results of this code as a table model for XSPEC that spans a range of physically relevant parameters. The parameters in their model are the luminosity of the jet, $L_{j}$; the Lorentz factor of the outflow, $\Gamma$; the fraction of kinetic energy dissipated, $\epsilon_{d}$; the fractions of 
dissipated energy going into electrons and magnetic field, $\epsilon_{B}$ and $\epsilon_{e}$; and the fraction of accelerated electrons that take on a power-law distribution, $\epsilon_{p l}$. This model has been used to fit the spectra of GRBs showing a prominent thermal bump, but has not been applied to ULGRBs.

Thus, we propose to fit the target ULGRBs with the subphotospheric model in XSPEC in order to determine the jet and dissipation parameters. We will compare the quality of the fit to other assumed models, such as a blackbody + power-law, to see whether a Comptonized blackbody offers any significant improvement in statistics. Even if this is not the case, as long as the photospheric model does not perform notably worse than other models, it could be considered preferable because it is physically motivated and gives information about the conditions in the jet. In addition, we will contrast the values of $\Gamma$ and $L_{j}$ inferred from detailed spectral modeling with the simple analytical constraints described above, to check whether the spectrum is consistent with my simple analytical picture for ULGRBs.

While the above studies give some idea of the type of jets that may be present in ULGRBs (and how they compare to standard GRBs), fitting the highly variable light curve is much more complicated. In the third phase, we will conduct hydrodynamical simulations of ultra-long jets, and then calculate the photospheric emission from these jets and compare the results with observations. Lazzati et al. (2013) already carried out a similar study for typical long GRBs; they found that the photosphere efficiently radiates the jet's kinetic energy and the light curve is strongly variable, qualitatively consistent with observations. However they did not consider extremely long engine durations or low jet luminosities as might be relevant to ULGRBs. In particular, a very long jet duration can considerably alter the dynamics, e.g. by significantly delaying the onset of deceleration. In addition, Lazzati et al. (2013) only considered 
high values for the terminal jet Lorentz factor $(>100)$, whereas the possibility exists that very long-duration engines have a smaller $\Gamma$. GRB 060218, for instance, is only mildly relativistic at late times (see Irwin \& Chevalier 2016). The results of phase one and two will come in handy here, as they will give some sense of the value of $\Gamma$ in ULGRBs.

We therefore propose to extend the modeling carried out by Lazzati et al. (2013) to the case of very long, somewhat low power, moderately relativistic jets, via 2D numerical simulations. This project would build upon the numerical work discussed in Chapter 3, exploring the higher-luminosity, long-timescale regime in the upper right of Fig. jetregimes. Post-processing capabilities will need to be added to RENZO in order to determine the location of the jet photosphere from the hydrodynamics, and to compute the resulting emission for observers on different lines of sight. (Alternately, a different code such as FLASH, which already includes the relevant radiation modules, could be used.) We will compare the numerically calculated light curves with observations, paying particular attention to whether the flaring behavior of ULGRBs, as well as the steep drop in the light curve after $10^{4} \mathrm{~s}$, can be reproduced.

\subsection{Expected results and applications}

The proposed research on ULGRBs could go one of two ways; in either case, the results would be interesting, and would lead to to provocative follow-up questions. On one hand, we may find that ULGRBs can in fact be well-explained by intrinsically long and low-luminosity engine models. Keeping in mind that the estimated global rate of ULGRBs is similar to the rate of typical GRBs, this result would be remarkable: it would suggest that a significant fraction of central engines release their energy over a relatively long timescale, $\sim 10^{4} \mathrm{~s}$. Combined with the results of Chapter 2 , this 
would imply not only that long-lived central engines are common, but also that longduration jets take on a wide range of luminosities. As with long-duration LLGRBs, the engine time is much longer than what is generally expected for a black-hole powered collapsar, so a paradigm shift in our understanding of GRB progenitors may be required to accommodate this result. In particular, ULGRBs may require a neutron star- or magnetar-driven engine to explain the long burst timescale. Comparing the energetics, radiative efficiency, Lorentz factor, and beaming factor of ULGRB jets to standard GRB jets could provide clues as to how and why ULGRB engines differ from the standard collapsar case. At the same time, a Comptonized-photosphere view of ULGRBs would establish an attractive connection between typical GRBs and their ultra-long counterparts via a common emission mechanism, which would naturally account for the features common to both classes such as light-curve variability and correlations between fundamental observables. This would perhaps suggest that the subphotospheric dissipation picture for cosmological GRBs is on the right track.

On the other hand, it might turn out that models with a long-lived dissipative jet are not well-suited to explaining ULGRB observations. This, too, would be interesting as it would indicate a gap in our understanding of the late phases of high-mass stellar evolution, with a substantial class of objects incompatible with either standard SN shock breakout or standard GRB collapsar models. In this case, a novel explanation for ULGRBs is needed. If an intrinsically long jet is not the answer, alternative scenarios such as nonstandard shock breakout, supergiant progenitor models, and low-density internal shock models may have to be revisited, or a new physical picture devised.

As Swift and optical survey facilities continue to uncover new and interesting transients, it is essential to be equipped with a comprehensive theory to interpret 
future observations of both ULGRBs and LLGRBs, especially with the next generation of specialized survey instruments on the horizon. With its current capabilities, Swift should find one new ULGRB every year or so. Our findings will not only be applicable to future Swift detections, but to future missions as well. For example, the proposed Explorer for Time-Domain Astrophysics (ETA) mission would offer improved sensitivity that would greatly enhance the detection rate of ULGRBs and other low-luminosity X-ray transients. ETA would operate in the $3-30 \mathrm{keV}$ range, making it particularly well-suited to ULGRBs and LLGRBs, where the peak energy is low and soft X-ray blackbodies are present.

As with LLGRBs, whether an extended circumstellar envelope plays a part in the very long X-ray emission timescale is an important open question. Unlike LLGRBs, however, the presence of a very long-lived and somewhat more luminous jet eliminates the possibility of jet choking, even if an extended envelope is present. Therefore, if the early optical emission in ULGRBs originates from a cooling envelope, it must be powered by the SN, and not by a choked jet. Optical surveys such as the intermediate Palomar Transient Factory (iPTF) and the upcoming Zwicky Transient Facility (ZTF)-which will offer an order-of-magnitude improvement in survey speed over PTF-may uncover additional broad-lined Type Ic SNe with early peaks, but establishing a convincing link between double-peaked SNe and ULGRBs may be difficult. ULGRBs are up to three orders of magnitude brighter than LLGRBs, and thus are seen out to much greater distances, making it much more challenging to detect and categorize the associated supernova. (However, this problem may be alleviated somewhat if ULGRB-associated SNe turn out to be very energetic, like the hypernovae associated with some standard GRBs, and the results of Greiner et al. (2015) suggest that this may be the case.) Thus, while the early optical peak seen in some 
ULGRBs is certainly suggestive of a nonstandard progenitor, it may take time and good luck to discover a nearby event that can be scrutinized to the same level of detail as GRB 060218 and similar LLGRBs. 


\section{Chapter 5}

\section{Summary and Conclusions}

Thanks to Swift, the zoo of $\gamma$-ray transients is growing more diverse every year. In this thesis, we investigated a variety of physics relevant to a recently discovered class of long-duration, low-luminosity GRBs. In Chapter 1, we opened with an overview of the relevant areas of GRB physics. Evidence connecting GRBs to the core collapse of massive stars was discussed, and the standard collapsar model was outlined. In this

model, a compact object formed from the collapse of the stellar core accretes infalling stellar material to power a relativistic jet. We described the propagation of this jet in a general sense, breaking down the possibilities into various regimes depending on whether the jet is collimated or uncollimated, the head is Newtonian or relativistic, the reverse shock is weak or strong, and the jet energy flows efficiently or inefficiently into the surrounding cocoon. The radiative mechanisms during the prompt phase were briefly reviewed in a qualitative way, with emphasis given to new observational and theoretical results that point towards the photospheric model of prompt emission. Finally, we discussed afterglow radiation in GRBs, with particular attention paid to the synchrotron emission from the external shocks.

In Chapter 2, an analytical model for GRB 060218 was presented. We went 
over observations of this unusual GRB, emphasizing differences from standard GRBs, but also highlighting some suggestive similarities such as obeying the Amati correlation. After a discussion of past analytical models of this burst and their various shortcomings, we argued that a standard relativistic shock breakout is unlikely to be responsible for the prompt emission in GRB 060218.

Next, a model accounting for each of the observed emission components was considered. The prompt thermal X-ray emission in GRB 060218 was described using the relativistic wind model of Paczynski (1990). We discussed how the early UV/optical could be generated by a shocked, low-mass envelope around the star, and applied the cooling envelope model of Nakar \& Piro (2014) to calculate the mass and radius of the envelope: $M_{\text {ext }} \sim 4 \times 10^{-3} M_{\odot}$ and $R_{\text {ext }} \sim 9 \times 10^{12} \mathrm{~cm}$. A dust echo model for the X-ray afterglow was presented that avoids the pitfalls of previous afterglow models for GRB 060218. We showed that the model reproduces the observed light curve and spectrum, and moreover that it does not require a high amount of circumburst dust.

The radio emission was modeled with the standard synchrotron afterglow model, although there was some tension with the timescale for the outflow to sphericize. We discussed how the choice of $\epsilon_{e}$ and $\epsilon_{B}$ affects the derived values of kinetic energy and circumburst density, and suggested that sweeping up some additional mass could solve the sphericization problem. Then, we considered the propagation of a low-luminosity jet through an extended envelope, and used several conditions to constrain the allowed value of the jet Lorentz factor and opening angle. Next, the inverse Compton radiation due to scattering the thermal X-ray photons off hot electrons in the external shocks was considered. The emission from the reverse shock was found to fit the data, if emission from the forward shock was suppressed by hand. The prompt emission model gave a jet isotropic energy of $E_{i s o} \sim 10^{53} \mathrm{ergs}$, and a jet Lorentz factor of $\sim 10$. 
The opening angle was constrained to be $\sim 0.1$, resulting in a jet energy similar to standard bursts. We inferred a high CSM density that we speculated was responsible for decelerating and widening the outflow prior to the onset of radio observations.

We compared our model with the alternative model of Nakar (2015), who modeled GRB 060218 as the shock breakout from a choked jet, and went over the strengths and weaknesses of each model in detail. Summarizing, both models predict an envelope, with roughly the same mass and radius. Both models predict a jet, although the jets have different properties. Nakar's model uses standard parameters for the jet, and is better at explaining the observed wide outflow at late times. However, it has difficulty producing the early optical emission, and also has difficulty producing the thermal X-ray component. Our model more naturally explains the fact that GRB 060218 obeys well-known GRB correlations. It does not have problems producing the thermal component or the early optical emission. However, it is only marginally consistent with the radio emission and requires a jet with unusual properties.

We also discussed a hybrid model that retains the best features of both our model and Nakar's. In this picture the prompt X-rays are produced by shock breakout, but the jet outflow that produces them is not spherical. The envelope is not destroyed by the jet's passage, so the optical can still be produced by interaction of the envelope with the SN. The jet energy mostly goes into the cocoon before breakout, so that the outflow is spherical in the CSM. Later, we found via numerical simulations that the physical conditions of the hybrid model (an outflow that breaks out from the envelope while still narrow, leaving most of the envelope intact, and then rapidly sphericizes outside the envelope) are generally easy to recreate for a range of physical parameters. We concluded that this is the best physical picture for GRB 060218 and other similar bursts. 
In Chapter 3, we discussed the results of our numerical simulations of jets in dense envelopes. We worked out an analytical picture that describes the degree of jet choking by the position in the $\xi-t_{j}$ plane, where $t_{j}$ is the jet duration and $\xi=L_{j} / \rho_{\text {ext }} R_{e x t}^{2} c^{3}$ is a dynamical parameter. Four different models were considered. In each model, the jet and star parameters corresponded to a typical GRB jet, and a typical LLGRB progenitor star. The envelope properties and the jet timescale were varied. We found more or less the same behavior of the jet while it is in the star as in previous studies. However, in all of our models, the outflow became essentially spherical shortly after breaking out of the envelope, which differs from the standard case of a bare star.

In the first model, we considered an envelope with the mass and radius derived in Chapter 2, and an $R^{-2}$ density profile. The second model was the same, but with a constant envelope density. The effect of this change on the jet breakout was found to be relatively minor. Both models were expected to be quasi-spherical at breakout based on analytical approximations. However, the numerical simulations showed instead that the outflow remained somewhat narrow. We speculated that the reason for this is that the cocoon shocks and slows the tail end of the jet material, reducing the rate at which energy flows into the cocoon at times $>t_{j}$.

Next, we considered a model with a high envelope mass, $M_{e x t}=0.2 M_{\odot}$. The envelope density in this case is sufficient to collimate the jet, and the outflow is even more narrow than in the first two models. However, even though all of the jet material catches up with the head at small radii, the ejecta remain narrow at breakout. The same is true of our final model, which investigates the effect of a larger envelope radius and a shorter jet duration. We discussed how physical limitations on the model parameters disfavor the optimal conditions for sphericizing the jet in 
the envelope. Based on our results, we concluded that the explosions in LLGRBs are likely not fully spherical at breakout, and went over the implications for event rates and shock breakout.

Finally, in Chapter 4, we talked about another recently discovered GRB subclass, ultra-long GRBs (ULGRBs), and considered how our previous work could be extended to investigate ULGRBs. ULGRBs have a lot in common with long-duration LLGRBs, including a thermal X-ray component, prompt optical emission, and a peculiar X-ray afterglow. Thus, many of the same physical ingredients, e.g. photospheric emission, extended envelope interaction, and X-ray light echoes from dust, may in the future prove useful for understanding ULGRBs as well.

LLGRBs are still a newly discovered phenomenon, and LLGRB theory is still in its nascent stages. Even so, discoveries so far indicate that the physics can be quite different from the standard GRB case. Clearly, there is still a lot left to learn about these objects, and about the many other newly uncovered classes of $\gamma$-ray transients. The rapid increase in our capability to observe and follow up on transient phenomena gives the sense that the next big discovery is always just around the corner. It is an exciting time to be doing transient astronomy.

I conclude by discussing a few more prospects for future work. Our group still has significant computational resources left over from the numerical work discussed in Chapter 3, which I will use to run several more models better exploring the $\xi-t_{j}$ parameter space. I remain highly interested in LLGRBs (and ULGRBs), but there is plenty of room in the world of high-energy astrophysics to branch out into other topics. One particular subject that has not gotten the theoretical attention it deserves is the interplay between the supernova and the jet in GRBs. Most simulations that have been performed so far include only a supernova, or only a jet, but it is easy to 
envision situations where including both could change the dynamics. For example, the dynamics may depend on the delay time between the SN and GRB (which is not known, and generally assumed to be 0). Also, in the case of low-power jets, the SN shock and the jet head have almost the same speed in the star, and they likely influence one another. For GRBs in extended envelopes, the way the supernova interacts with the aspherically distributed matter left behind after the jet crosses the envelope is also worth investigating. Some other topics of interest to me include supernova shock breakout and the structure of radiative shocks; tidal disruption events; and other newly realized optical/infrared transients such as kilonovae. 
Appendices 


\section{Appendix A}

\section{Shock Dynamics of a Relativistic \\ Outflow Interacting with a}

\section{Power-Law CSM}

Analytical solutions of equations (2.20)-(2.25) are available when $\gamma \gg 1$. We consider the general case where the outer density profile is a power law in radius as in equation (2.25), and the luminosity of thermal photons and the kinetic luminosity of the jet vary as power laws in time, i.e. $L_{t h}=L_{0}\left(t / t_{L}\right)^{k}$ and $L_{i s o}=\mathcal{L}_{0}\left(t / t_{M}\right)^{s}$. We therefore have $M_{i s o}\left(t_{\text {emit }}\right) \approx L_{\text {iso }} \gamma^{-1} c^{-2}\left(t_{\text {emit }} / t\right)^{s}$. Combining equations (2.20), (2.24), and (2.25) with the above expressions leads to

$$
f=C_{0} A_{*}^{-1} L_{i s o, 48} \gamma^{-2}\left(\frac{t_{\text {emit }}}{t}\right)^{s}\left(\frac{R}{R_{\text {ext }}}\right)^{\alpha-2}\left(\frac{R_{f s}}{R_{r s}}\right)^{2} .
$$

$C_{0}=5.9 \times 10^{3}$ is a dimensionless constant determined by scaling the density to $A_{*}$ and $L_{i s o}$ to $10^{48} \mathrm{ergs} \mathrm{s}^{-1}$. Note that, in our model, $L_{t h}$ and $L_{i s o}$ are related by equation 
(2.3); when $\gamma \gg 1$ we have

$$
L_{i s o, 48} \approx 2.3 \times 10^{-3} \xi^{-2} L_{t h, 46}^{1 / 2} \gamma^{4}
$$

For the sake of convenience and generality we do not make this substitution yet.

Three dynamical limits are possible, depending on the relative value of $f$ and $\gamma$ (Sari \& Piran 1995):

1. The coasting regime $\left(f \gg \gamma^{2}\right)$ : The forward shock (FS) coasts with an approximately constant Lorentz factor, and the RS is Newtonian with $\bar{\beta}_{3} \ll 1$ :

$$
\begin{aligned}
& \gamma_{2} \approx \gamma \\
& \bar{\beta}_{3} \approx\left(\frac{8 \gamma^{2}}{7 f}\right)^{1 / 2} \\
& R=\frac{\beta_{2} c t}{1-\beta_{2}} \approx 2 \gamma^{2} c t \\
& t_{\text {emit }}=\frac{\beta-\beta_{2}}{\beta\left(1-\beta_{2}\right)} t \approx 2 \bar{\beta}_{3} t .
\end{aligned}
$$

The shocked regions are thin; the forward shock's size is $\sim R / \gamma^{2}$, and the reverse shock is even thinner by a factor $\bar{\beta}_{3}$, so that $R_{r s} \approx R \approx R_{f s}$ is a good approximation.

2. The decelerating (or accelerating) regime $\left(\gamma^{-2} \ll f \ll \gamma^{2}\right)$ : The FS and the RS are both relativistic:

$$
\begin{aligned}
& \gamma_{2} \approx\left(\frac{f \gamma^{2}}{4}\right)^{1 / 4} \\
& \bar{\gamma}_{3} \approx\left(\frac{\gamma^{2}}{4 f}\right)^{1 / 4} \\
& R \approx 2 \gamma_{2}^{2} c t \\
& t_{\text {emit }} \approx t .
\end{aligned}
$$


Accelerating or decelerating cases are possible, depending on the evolution of $f$. As in the coasting case, the shocked regions are thin compared to their radius, so that $R_{r s} \approx R \approx R_{f s}$ applies.

3. The non-relativistic regime $\left(f \ll \gamma^{-2}\right)$ : A third solution is also possible in which the FS becomes non-relativistic and $R_{r s} \ll R_{f s}$. As the requisite high CSM density and low engine Lorentz factor are unlikely to be encountered in GRBs, we do not discuss this scenario further.

The CSM density $\rho_{1} \propto R^{-\alpha} \propto\left(\gamma_{2}^{2} t\right)^{-\alpha}$ depends on time implicitly through $\gamma_{2}$. It is useful to separate out the explicit time dependence by defining $B_{*}=A_{*}\left(t / t_{\text {ext }}\right)^{2-\alpha}$, with $t_{\text {ext }} \equiv R_{\text {ext }} / c$. Additionally, we make the convenient definition $\ell=2-\alpha$. Then, by substituting equation (A.1) into equation (A.3) or (A.4), one obtains solutions for the dynamical variables after some algebra:

$$
\begin{gathered}
f=\left\{\begin{array}{lc}
{\left[C_{1} L_{i s o, 48} B_{*}^{-1} \gamma^{-(\ell+2)+(s-\ell)}\right]^{2 /(s+2)},} & f>\gamma^{2} \\
{\left[C_{0} L_{i s o, 48} B_{*}^{-1} \gamma^{-(\ell+2)}\right]^{2 /(\ell+2)},} & \gamma^{-2}<f<\gamma^{2}
\end{array},\right. \\
\gamma_{2}=\left\{\begin{array}{lc}
\gamma, & f>\gamma^{2} \\
2^{-1 / 2}\left[C_{0} L_{i s o, 48} B_{*}^{-1}\right]^{1 / 2(\ell+2)}, & \gamma^{-2}<f<\gamma^{2}
\end{array}\right.
\end{gathered}
$$

and

$$
\bar{\beta}_{3} \bar{\gamma}_{3}=\left\{\begin{array}{cc}
\left(\frac{8}{7}\right)^{1 / 2}\left[C_{1} L_{i s o, 48} B_{*}^{-1} \gamma^{-2(\ell+2)}\right]^{-1 /(s+2)}, & f>\gamma^{2} \\
2^{-1 / 2}\left[C_{0} L_{i s o, 48} B_{*}^{-1} \gamma^{-2(\ell+2)}\right]^{-1 / 2(\ell+2)}, & \gamma^{-2}<f<\gamma^{2}
\end{array} .\right.
$$

We have defined $C_{1}=(32 / 7)^{s / 2} 2^{-\ell} C_{0}$, as this quantity appears repeatedly. We see that the essential dynamical variables all depend on $B_{*}^{-1} L_{i s o} \propto t^{s-\ell}$, allowing for two possibilities. If $s<\ell$ we have the typical case where the forward shock begins in a 
coasting state and starts to decelerate once $f \sim \gamma^{2}$. If $s>\ell$, however, the forward shock starts with $\gamma_{2}<\gamma$ and accelerates until it reaches a terminal Lorentz factor $\gamma$ when $f \sim \gamma^{2}$. When $s=\ell$, the shock velocity is constant in time; this special case generalizes the result of Emmering \& Chevalier (1987), who studied a constant luminosity outflow $(s=0)$ in a wind density profile $(\ell=0)$. The usual afterglow dynamics (e.g., Sari \& Piran 1995; Sari et al. 1998) can be recreated with $s=-1$ (negligible energy input) and $\ell=2$ (constant density CSM). The transition between dynamical regimes occurs when $f \simeq \gamma^{2}$, at an approximate time

$$
t_{f} \simeq\left[C_{0}^{-1} \mathcal{L}_{0,48}^{-1} A_{*} \gamma^{2(\ell+2)} t_{e x t}^{-\ell} t_{M}^{s}\right]^{1 /(s-\ell)}
$$

although the exact time of transition differs for $f, \gamma_{2}$, and $\bar{\gamma}_{3}$ due to different leading numerical factors.

To obtain the spectral parameters in Appendix B, it is useful to have expressions for $N_{2}$ and $N_{3}$, the number of electrons contained in regions 2 and 3. The number of electrons swept into the forward shock can be found by integrating over the CSM density profile: $N_{2}=\chi_{e} \int_{0}^{R} 4 \pi r^{2} \rho_{1} d r / m_{p}$, where $m_{p}$ is the proton mass and $\chi_{e}$ is the average number of electrons per nucleon. We take $\chi_{e}=0.5$, appropriate for hydrogen-free gas, which leads to

$$
N_{2}=\frac{5.6 \times 10^{49}}{(\ell+1)} \times\left\{\begin{array}{lr}
2^{\ell+1} B_{*} \gamma^{2(\ell+1)} t_{3}, & f>\gamma^{2} \\
{\left[C_{0}^{\ell+1} L_{i s o, 48}^{\ell+1} B_{*}\right]^{1 /(\ell+2)} t_{3},} & \gamma^{-2}<f<\gamma^{2}
\end{array} .\right.
$$

$t_{3}$ is the time in units of $10^{3} \mathrm{~s}$. The number of electrons in the reverse-shocked region 
is $N_{3}=\chi_{e} \int_{0}^{t_{e m i t}} L_{i s o} \gamma^{-1} d t / m_{p} c^{2}$, which gives

$N_{3}=\frac{5.6 \times 10^{49}}{(s+1)} \times\left\{\begin{array}{lr}2^{\ell}\left(\frac{32}{7}\right)^{1 / 2}\left[C_{1} L_{i s o, 48} B_{*}^{s+1} \gamma^{2(\ell+2)(s+1)-(s+2)}\right]^{1 /(s+2)} t_{3}, & f>\gamma^{2} \\ C_{0} L_{i s o, 48} \gamma^{-1} t_{3}, & \gamma^{-2}<f<\gamma^{2}\end{array}\right.$.

Note that $N_{3} \sim f^{1 / 2} N_{2}$. The comoving optical depth of region 2 can be estimated as $\tau_{2} \sim\left(\sigma_{T} N_{2}\right) /\left(4 \pi R^{2}\right)$, and likewise for region 3 . 


\section{Appendix B}

\section{Inverse Compton Radiation from a} Relativistic Outflow Interacting

\section{with a Power-Law CSM}

The FS and RS, if at least mildly relativistic, will produce relativistic electrons and strong magnetic fields that give rise to nonthermal emission. We adopt the standard theory, wherein fractions $\epsilon_{e}$ and $\epsilon_{B}$ of the total postshock energy density go into relativistic electrons and magnetic fields, respectively. The postshock electron energies are assumed to be distributed as a power law, $N_{\gamma_{e}} \propto\left(\gamma_{e}-1\right)^{-p}$, above some minimum Lorentz factor $\gamma_{m}$. We have $\gamma_{m 2}=610 \epsilon_{e 2} g_{p}\left(\gamma_{2}-1\right)$ and $\gamma_{m 3}=610 \epsilon_{e 3} g_{p}\left(\bar{\gamma}_{3}-1\right)$ for regions 2 and 3, respectively (e.g., Sari et al. 1998). $g_{p}=3(p-2) /(p-1)$ scales the results to $p=2.5$.

Let $P\left(\gamma_{e}\right)$ be the power radiated by a relativistic electron, and $\nu\left(\gamma_{e}\right)$ be the frequency of that radiation. We have $P\left(\gamma_{e}\right)=(4 / 3) \sigma_{T} c \gamma_{e}^{2} \gamma_{2}^{2} u_{r a d}$, where $\sigma_{T}$ is the Thomson cross section and $u_{r a d}=L_{t h} /\left(4 \pi R^{2} \gamma_{2}^{2} c\right)$ is the photon energy density in the comoving frame. An electron with $\gamma_{e}$ emits at frequency $\nu\left(\gamma_{e}\right)=\gamma_{2} \gamma_{e}^{2} \nu_{\text {rad }}$, where $\nu_{\text {rad }} \sim$ 
$k_{B} T_{0} / h \gamma_{2}$ is the frequency of a typical thermal photon in the shock frame (Rybicki \& Lightman 1979). Electrons above the critical Lorentz factor $\gamma_{c}=\left(3 m_{e} c\right) /\left(4 \sigma_{T} \gamma_{2} u_{\text {rad }} t\right)$ can cool in time $t$ (Sari et al. 1998; Dai et al. 2006). $\gamma_{c}$ is the same for regions 2 and 3 because the energy density and bulk Lorentz factor are equal across the contact discontinuity. In the single scattering limit, the maximum spectral power emitted by an ensemble of $N_{e}$ electrons will be $L_{\nu, \max } \approx N_{e} P\left(\gamma_{e}\right) / \nu\left(\gamma_{e}\right)$ (Sari et al. 1998; Dai et al. 2006). Ignoring the self-absorption frequency $\nu_{a}$, which falls well below the X-ray band, the spectrum will have two breaks, at $\nu_{c}=\nu\left(\gamma_{c}\right)$ and $\nu_{m}=\nu\left(\gamma_{m}\right)$, and two possible shapes depending on whether $\nu_{m}<\nu_{c}$ (slow cooling) or $\nu_{c}<\nu_{m}$ (fast cooling). The form of $L_{\nu}$ in either case is given by equations (7) and (8) in Sari et al. (1998).

If the above expressions give $\gamma_{m 2}<1, \gamma_{m 2} \approx 1$ should be used. However, in that case, only a fraction $N_{\text {rel }} / N_{2} \approx\left[610 \epsilon_{e 2} g_{p}\left(\gamma_{2}-1\right)\right]^{p-1}$ of the electrons are relativistic with $\gamma_{e}-1 \geq 1$. We make the approximation that non-relativistic electrons do not contribute significantly to the emission at $\nu>k T_{0}$. Then the spectrum above $\nu_{m 2}$, where $L_{\nu} \propto L_{\nu, \max 2} \nu_{m 2}^{(p-1) / 2} \propto N_{\text {rel }} \gamma_{m 2}^{p-1}$, is unchanged whether $\gamma_{m 2}>1$ or $\gamma_{m 2} \approx 1$. The same applies for $\gamma_{m 3}$.

With the above assumptions and the dynamical equations of Appendix A, one can compute the spectral parameters. For IC, the characteristic frequencies in region 2 and 3 are

$$
h \nu_{m 2}=630 \mathrm{keV} \times\left\{\begin{array}{lr}
g_{p}^{2} \epsilon_{e 2,-1}^{2} \xi \gamma^{2}, & f>\gamma^{2} \\
\frac{1}{2} g_{p}^{2} \epsilon_{e 2,-1}^{2} \xi\left[C_{0} L_{i s o, 48} B_{*}^{-1}\right]^{1 /(\ell+2)}, & \gamma^{-2}<f<\gamma^{2}
\end{array} .\right.
$$


and

$$
h \nu_{m 3}=630 \mathrm{keV} \times\left\{\begin{array}{lc}
\left(\frac{4}{7}\right)^{2} g_{p}^{2} \epsilon_{e 3,-1}^{2} \xi\left[C_{1} L_{i s o, 48} B_{*}^{-1} \gamma^{-2(\ell+2)}\right]^{-4 /(s+2)}, & f>\gamma^{2} \\
\frac{1}{2} g_{p}^{2} \epsilon_{e 3,-1}^{2} \xi\left[C_{0} L_{i s o, 48} B_{*}^{-1} \gamma^{-2(\ell+2)}\right]^{-1 /(\ell+2)}, & \gamma^{-2}<f<\gamma^{2}
\end{array}\right.
$$

Assuming IC is the dominant cooling process, the cooling frequency is

$$
h \nu_{c}=3.0 \times 10^{-6} \mathrm{keV} \times\left\{\begin{array}{lr}
L_{t h, 46}^{-2} \xi \gamma^{10} t_{3}^{2}, & f>\gamma^{2} \\
\frac{1}{32} L_{t h, 46}^{-2} \xi\left[C_{0} L_{i s o, 48} B_{*}^{-1}\right]^{5 /(\ell+2)} t_{3}^{2}, & \gamma^{-2}<f<\gamma^{2}
\end{array}\right.
$$

The peak IC spectral power in region 2 (in cgs units) is

$$
L_{\nu, \max 2}=\frac{1.1 \times 10^{27}}{(\ell+1)} \times\left\{\begin{array}{lc}
2^{\ell-1} L_{t h, 46} \xi^{-1} B_{*} \gamma^{2(\ell-1)} t_{3}^{-1}, & f>\gamma^{2} \\
L_{t h, 46} \xi^{-1}\left[C_{0}^{\ell-1} L_{i s o, 48}^{\ell-1} B_{*}^{3}\right]^{1 /(\ell+2)} t_{3}^{-1}, & \gamma^{-2}<f<\gamma^{2}
\end{array}\right.
$$

and in region 3 it is

$$
L_{\nu, \max 3}=\frac{1.1 \times 10^{27}}{(s+1)} \times\left\{\begin{array}{lr}
2^{\ell-2}\left(\frac{32}{7}\right)^{1 / 2} L_{t h, 46} \xi^{-1} \\
\times\left[C_{1} L_{i s o, 48} B_{*}^{s+1} \gamma^{2(\ell+2)(s+1)-5(s+2)}\right]^{1 /(s+2)} t_{3}^{-1}, & f>\gamma^{2} \\
L_{t h, 46} \xi^{-1}\left[C_{0}^{\ell} L_{i s o, 48}^{\ell} B_{*}^{2} \gamma^{-(\ell+2)}\right]^{1 /(\ell+2)} t_{3}^{-1}, & \gamma^{-2}<f<\gamma^{2}
\end{array}\right.
$$

Table B.1 summarizes the time behaviour of the spectral parameters. We point out that, for $s>\ell, \nu_{m}$ tends towards a steep decay $\propto t^{-4(s-\ell) /(s+2)}$ as the coasting regime is approached, making this model particularly well-suited to describing GRB 060218 or other objects where a rapid decline in $E_{p}$ is observed.

Given equations (B.1)-(B.5), the spectrum of the forward (reverse) shock emission can be constructed for any ordering of $\nu_{c}, \nu_{m 2}\left(\nu_{m 3}\right)$, and the observed frequency $\nu$ according to Sari et al. (1998). If the flux density at $\nu$ and the peak energy are 
measured at $t \ll t_{f}$ or $t \gg t_{f}$, and the relationship between $\nu, \nu_{c}$, and $\nu_{m}$ is known, then we can invert the model expressions for flux and peak energy to solve for two of the parameters $\gamma, A_{*}$, and $\epsilon_{e}$ in terms of observables and the third parameter. As a practical example, consider the case where the reverse shock dominates the emission at $t \ll t_{f}$ and $\nu_{c}<\nu<\nu_{m 3}$. In this limit we have $L_{\nu}=L_{\nu, \max 3} \nu_{c}^{1 / 2} \nu^{-1 / 2}$ and $E_{p}=\nu_{m 3}$; $\nu_{m 3}, \nu_{c}$ and $L_{\nu, \max 3}$ are given by equations (B.2), (B.3), and (B.5) respectively, taking the solution for $f<\gamma^{2}$. The luminosity integrated over a frequency range $\nu_{1}-\nu_{2}$ (with $\nu_{c}<\nu_{1}$ and $\left.\nu_{2}<\nu_{m 3}\right)$ is

$$
L_{i n t}=8.1 \times 10^{40} \mathrm{erg} \mathrm{s}^{-1}(s+1)^{-1} C_{i n t} \xi^{-1 / 2}\left[C_{1}^{2 \ell+5} L_{i s o, 48}^{2 \ell+5} B_{*}^{-1}\right]^{1 / 2(\ell+2)} \gamma^{-1},
$$

where $C_{\text {int }}=2\left[\left(h \nu_{2} / \mathrm{keV}\right)-\left(h \nu_{1} / \mathrm{keV}\right)\right]$. Defining two dimensionless quantities that depend on the observables, $\tilde{L}_{i n t}=L_{i n t} /\left(8.1 \times 10^{40} \mathrm{erg} \mathrm{s}^{-1}\right)$ and $\tilde{E}_{p}=E_{p} /(7.6 \times$ $10^{19} \mathrm{~Hz}$ ), considerably simplifies the algebra. Equations (B.2) and (B.6) can be rewritten to eliminate either $B_{*}$ or $L_{i s o}$ :

$$
\tilde{L}_{\text {int }}^{2} \tilde{E}_{p}=(s+1)^{-2} C_{\text {int }}^{2}\left(g_{p} \epsilon_{e 3,-1}\right)^{2}\left(C_{0} L_{i s o, 48}\right)^{2}
$$

\begin{tabular}{|l|c|c|}
\hline Quantity & Coasting $\left(f>\gamma^{2}\right)$ & Accelerating $\left(f<\gamma^{2}\right)$ \\
\hline$\nu_{m 2}$ & 0 & $\frac{s-\ell}{\ell+2}$ \\
\hline$\nu_{m 3}$ & $\frac{-4(s-\ell)}{s+2}$ & $\frac{-(s-\ell)}{\ell+2}$ \\
\hline$\nu_{c}$ & $-2(k-1)$ & $-2(k-1)+\frac{5(s-\ell)}{\ell+2}$ \\
\hline$L_{\nu, \max 2}$ & $(k-1)+\ell$ & $(k-1)+s-\frac{3(s-\ell)}{\ell+2}$ \\
\hline$L_{\nu, \max 3}$ & $(k-1)+\ell+\frac{s-\ell}{s+2}$ & $(k-1)+s-\frac{2(s-\ell)}{\ell+2}$ \\
\hline
\end{tabular}

Table B.1: Temporal evolution of the spectral parameters. Each parameter in the leftmost column evolves as a power-law in time, with the power-law index in the coasting and accelerating regimes given in the centre and right columns, respectively. 
and

$$
\tilde{L}_{i n t} \tilde{E}_{p}^{(2 \ell+5) / 2}=(s+1)^{-1} C_{i n t}\left(g_{p} \epsilon_{e 3,-1}\right)^{2 \ell+5} \xi^{\ell+2} B_{*} \gamma^{2(\ell+2)} .
$$

Finally, if the thermal photons come from a dissipative outflow as described in Section 2.4.1, we can substitute equation (A.2) for $L_{i s o, 48}$ and solve for $\gamma$ and $B_{*}$, with the results

$$
\gamma=(13.6)^{-1 / 4}(s+1)^{1 / 4} C_{\text {int }}^{-1 / 4}\left(g_{p} \epsilon_{e 3,-1}\right)^{-1 / 4} L_{\text {th,46 }}^{-1 / 8} \xi^{1 / 2} \tilde{L}_{\text {int }}^{1 / 4} \tilde{E}_{p}^{1 / 8}
$$

and

$$
B_{*}=(13.6)^{(\ell+2) / 2}(s+1)^{-\ell / 2} C_{i n t}^{\ell / 2}\left(g_{p} \epsilon_{e 3,-1}\right)^{-(3 \ell+8) / 2} L_{t h, 46}^{(\ell+2) / 4} \xi^{-2(\ell+2)} \tilde{L}_{\text {int }}^{-\ell / 2} \tilde{E}_{p}^{(3 \ell+8) / 4} .
$$

In GRB 060218, we have $s=k / 2$ by equation (2.3), and since $k=0.66$ (Liang et al. 2006), $s=0.33$. We can estimate $\alpha$ and $p$ by assuming the coasting regime has been reached by late times. We have $\nu_{m 3} \propto t^{-4(s-\ell) /(s+2)}$ for $f>\gamma^{2}$, so in order to get $E_{p} \propto \nu_{m 3} \propto t^{-1.6}$ we require $\ell=-0.6$ and $\alpha=2.6$. This in turn gives $\nu_{c} \propto t^{0.68}$ and $L_{\nu, \max 3} \propto t^{-0.54}$ for $t \gg t_{f}$. To obtain a light curve $L_{\nu, \max 3} \nu_{c} \nu_{m 3}^{(p-1) / 2} \propto t^{-2}$ at high energies, $p=3.25$ is needed, implying $g_{p}=1.67$. The corresponding high energy spectral index is $\beta_{2} \approx-1.6$, consistent with the data of Toma et al. (2007). Taking the same values for $\alpha$ and $s$, we have $L_{\nu, \max 3} \propto t^{-1.33}$ and $\nu_{c} \propto t^{4}$ at early times when $f<\gamma^{2}$. In this limit the light curve for $\nu_{c}<\nu<\nu_{m}$ goes as $L_{\nu, \max 3} \nu_{c}^{1 / 2} \propto$ $t^{0.67}$, consistent with the early rise in the XRT and BAT light curves. We conclude that $t<t_{f}$ at early times, and since the $0.3-10 \mathrm{keV}$ band is well below $E_{p}$ at early times (and presumably above $h \nu_{c}$ ), we can apply the model described above. Taking $L_{\text {int }}=L_{X R T}$, we calculate $C_{B A T}=5.23$. At $t=300 \mathrm{~s}$, the $0.3-10 \mathrm{keV}$ luminosity was $L_{X R T} \approx 1 \times 10^{46} \mathrm{erg} \mathrm{s}^{-1}$ (Campana et al. 2006) and the peak energy was $E_{p} \approx 23 \mathrm{keV}$ (Toma et al. 2007). Thus, we have $\tilde{L}_{i n t}=1.2 \times 10^{5}$ and $\tilde{E}_{p}=7.3 \times 10^{-2}$. Finally, 
we have $L_{t h, 46} \approx 0.2$ and $\xi \approx 1$ at $300 \mathrm{~s}$ (Campana et al. 2006), $t_{M}=t_{L} \approx 2800 \mathrm{~s}$ (Campana et al. 2006), and $t_{\text {ext }}=300 \mathrm{~s}$ (Section 2.4.3) so $B_{*} \approx A_{*}$ at $300 \mathrm{~s}$. Plugging all of this into equations (B.9) and (B.10), we find

$$
\gamma=5.3 \epsilon_{e 3,-1}^{-1 / 4}
$$

and

$$
A_{*}=0.28 \epsilon_{e 3,-1}^{-(3 \ell+8) / 2} \text {. }
$$

When $\epsilon_{e 3}=10^{-2.5}$, we obtain $\gamma \approx 13$ and $A_{*} \approx 1.3 \times 10^{4}$, reasonably close to the results of the best-fitting numerical model.

We stress that this model is not self-consistent unless IC is more important than synchrotron, and the emission is dominated by the RS. Here we check whether each of these conditions is satisfied. With $\gamma$ and $A_{*}$ as above, we calculate $L_{i s o, 48} \approx 26$, $f \approx 0.36, \gamma_{2} \approx 1.9, \bar{\gamma}_{3} \approx 1.7, R \approx 6.5 \times 10^{13} \mathrm{~cm}$, and $\rho_{1}(R) \approx 4.7 \times 10^{-13} \mathrm{~g} \mathrm{~cm}^{-3}$ at $300 \mathrm{~s}$. The comoving energy density of thermal photons at the shock radius is $u_{\text {rad }}=L_{t h} / 4 \pi R^{2} \gamma_{2}^{2} c=3.5 \times 10^{5} \mathrm{erg} \mathrm{cm}^{-3}$, while the energy density in magnetic fields is $u_{B}=4 \gamma_{2}^{2} \rho_{1} c^{2} \epsilon_{B}=6.1 \times 10^{9} \epsilon_{B} \mathrm{erg} \mathrm{cm}^{-3}$ (Sari et al. 1998). Synchrotron is not too important if $u_{B} / u_{\text {rad }} \lesssim 1$, implying $\epsilon_{B} \lesssim 6 \times 10^{-5}$, similar to the numerically inferred value.

We have assumed $\nu_{c}<\nu<\nu_{m 3}$, so that the RS luminosity is $L_{R S}=L_{\nu, \max 3} \nu_{c}^{1 / 2} \nu^{-1 / 2}$. Whether the FS or RS dominates the emission depends on the value of $\nu_{m 2}$. If $\nu_{m 2}>\nu$, then $L_{R S} / L_{F S}=\left(L_{\nu, \max 3} \nu_{c}^{1 / 2} \nu^{-1 / 2}\right) /\left(L_{\nu, \max 2} \nu_{c}^{1 / 2} \nu^{-1 / 2}\right)=L_{\nu, \max 3} / L_{\nu, \max 2} \sim N_{3} / N_{2} \sim$ $f^{1 / 2}$. This cannot be the case, since $f<1$ at $300 \mathrm{~s}$ in our model. Instead, we require $\nu_{m 2}<\nu$, so that $L_{F S}=L_{\nu, \max 2} \nu_{c}^{1 / 2} \nu_{m 2}^{(p-1) / 2} \nu^{-p / 2}$, and $L_{R S} / L_{F S} \sim f^{1 / 2}\left(\nu / \nu_{m 2}\right)^{(p-1) / 2}$. Substituting $h \nu_{m 2} \simeq\left[610 g_{p} \epsilon_{e 2}\left(\gamma_{2}-1\right)\right]^{2} k T_{0}$, we obtain $L_{R S} / L_{F S} \gtrsim 1$ when $\epsilon_{e 2} \lesssim$ 
$9 \times 10^{-4}\left(h \nu / k T_{0}\right)^{1 / 2}$. This is qualitatively similar to the numerical result in that it also suggests $\epsilon_{e 2}<\epsilon_{e 3}$, although the numerical model produced a tighter upper limit on $\epsilon_{e 2}$. 


\section{References}

Ahlgren, B., Larsson, J., Nymark, T., Ryde, F., \& Pe'er, A. 2015, MNRAS, 454, L31

Aloy, M. A., Janka, H.-T., Müller, E. 2005, A\&A, 436, 273

Aloy, M. A., Ibáñez, J. M., Martí, J. M., Gómez, J.-L., Müller, E. 1999, ApJ, 523, L125

Amati, L., Frontera, F., Tavani, M., et al. 2002, A\&A, 390, 81

Amati, L. 2006, MNRAS, 372, 233

Axelsson, M., \& Borgonovo, L. 2015, MNRAS, 447, 3150

Band, D., Matteson, J., Ford, L., et al. 1993, ApJ, 413, 281

Bannister, K., Hancock, P., Kulkarni, S., et al. 2013, The Astronomer's Telegram, 5531,1

Barniol Duran, R., Nakar, E., \& Piran, T. 2013, ApJ, 772, 78

Barniol Duran, R., Nakar, E., Piran, T., \& Sari, R. 2015, MNRAS, 448, 417

Bartos, I., Dasgupta, B., \& Márka, S. 2012, Phys. Rev. D, 86, 083007

Begelman, M. C., \& Cioffi, D. F. 1989, ApJ, 345, L21 
Bellm, E. C., Barrière, N. M., Bhalerao, V., et al. 2014, ApJL, 784, L19

Björnsson, C.-I. 2008, ApJ, 672, 443

Blandford, R. D., \& McKee, C. F. 1976, Physics of Fluids, 19, 1130

Blandford, R. D., \& Znajek, R. L. 1977, MNRAS, 179, 433

Bromberg, O., \& Levinson, A. 2009, ApJ, 699, 1274

Bromberg, O., Nakar, E., \& Piran, T. 2011, ApJ, 739, L55

Bromberg, O., Nakar, E., Piran, T., \& Sari, R. 2011, ApJ, 740, 100

Bryan, G. L., Norman, M. L., O'Shea, B. W., et al. 2014, ApJS, 211, 19

Burgess, J. M., Preece, R. D., Ryde, F., et al. 2014, ApJ, 784, L43

Campana, S., Mangano, V., Blustin, A. J., et al. 2006, Nature, 442, 1008

Cano, Z., Bersier, D., Guidorzi, C., et al. 2011, ApJ, 740, 41

Cenko, S. B., Krimm, H. A., Horesh, A., et al. 2012, ApJ, 753, 77

Chevalier, R. A. 1992, ApJ, 394, 559

Chevalier, R. A., \& Li, Z.-Y. 1999, ApJ, 520, L29

Chevalier, R. A., \& Li, Z.-Y. 2000, ApJ, 536, 195

Chevalier, R. A., \& Fransson, C. 2008, ApJ, 683, L135

Chevalier, R. A. 2012, ApJ, 752, L2

Chhotray, A., \& Lazzati, D. 2015, ApJ, 802, 132 
Chornock, R., Berger, E., Levesque, E. M., et al. 2010, arXiv:1004.2262

Christensen, L., Hjorth, J., \& Gorosabel, J. 2004, A\&A, 425, 913

Costa, E., Frontera, F., Heise, J., et al. 1997, Nature, 387, 783

Couch, S. M., Wheeler, J. C., \& Milosavljević, M. 2008, New Horizons in Astronomy, 393,183

Dai, Z. G., Zhang, B., \& Liang, E. W. 2006, arXiv:astro-ph/0604510

Draine, B. T., \& Bond, N. A. 2004, ApJ, 617, 987

Drout, M. R., Milisavljevic, D., Parrent, J., et al. 2016, ApJ, 821, 57

Emmering, R. T., \& Chevalier, R. A. 1987, ApJ, 321, 334

Evans, P. A., Willingale, R., Osborne, J. P., et al. 2014, MNRAS, 444, 250

Fan, Y.-Z., Piran, T., \& Xu, D. 2006, JCAP, 9, 13

Fan, Y.-Z., Zhang, B.-B., Xu, D., Liang, E.-W., \& Zhang, B. 2011, ApJ, 726, 32

Fan, Y.-Z., Wei, D.-M., Zhang, F.-W., \& Zhang, B.-B. 2012, ApJL, 755, L6

Fox, O. D., Silverman, J. M., Filippenko, A. V., et al. 2015, MNRAS, 447, 772

Frail, D. A., Kulkarni, S. R., Nicastro, L., Feroci, M., \& Taylor, G. B. 1997, Nature, 389,261

Fransson, C., Ergon, M., Challis, P. J., et al. 2014, ApJ, 797, 118

Friis, M., \& Watson, D. 2013, ApJ, 771, 15

Galama, T. J., Vreeswijk, P. M., van Paradijs, J., et al. 1998, Nature, 395, 670 
Gendre, B., Stratta, G., Atteia, J. L., et al. 2013, ApJ, 766, 30

Ghirlanda, G., Bosnjak, Z., Ghisellini, G., Tavecchio, F., \& Firmani, C. 2007, MNRAS, 379,73

Ghisellini, G., Ghirlanda, G., \& Tavecchio, F. 2007, MNRAS, 375, L36

Ghisellini, G., Ghirlanda, G., \& Tavecchio, F. 2007, MNRAS, 382, L77

Gorbikov, E., Gal-Yam, A., Ofek, E. O., et al. 2014, MNRAS, 443, 671

Granot, J., Piran, T., \& Sari, R. 2000, ApJ, 534, L163

Granot, J., \& Sari, R. 2002, ApJ, 568, 820

Greiner, J., Krühler, T., Klose, S., et al. 2011, A\&A, 526, A30

Greiner, J., Mazzali, P. A., Kann, D. A., et al. 2015, Nature, 523, 189

Guenther, E. W., Klose, S., Vreeswijk, P., Pian, E., \& Greiner, J. 2006, GRB Coordinates Network, 4863, 1

Guetta, D., \& Della Valle, M. 2007, ApJ, 657, L73

Güver, T., \& Özel, F. 2009, MNRAS, 400, 2050

Hancock, P. J., Murphy, T., Gaensler, B., \& Zauderer, A. 2012, GRB Coordinates Network, 12804, 1

Woosley, S. E., \& Heger, A. 2006, ApJ, 637, 914

Holland, S. T., Sbarufatti, B., Shen, R., et al. 2010, ApJ, 717, 223

Horesh, A., Cenko, S. B., Perley, D. A., et al. 2015, ApJ, 812, 86 
Horiuchi, S., \& Ando, S. 2008, Phys. Rev. D, 77, 063007

Hughes, P. A., Miller, M. A., \& Duncan, G. C. 2002, ApJ, 572, 713

Irwin, C. M., \& Chevalier, R. A. 2016, MNRAS, 460, 1680

Ito, H., Nagataki, S., Ono, M., et al. 2013, ApJ, 777, 62

Kaneko, Y., Ramirez-Ruiz, E., Granot, J., et al. 2007, ApJ, 654, 385

Katz, B., Budnik, R., \& Waxman, E. 2010, ApJ, 716, 781

Katz, J. I. 1994, ApJ, 432, L107

Katz, J. I., \& Piran, T. 1997, ApJ, 490, 772

Klebesadel, R. W., Strong, I. B., \& Olson, R. A. 1973, ApJ, 182, L85

Komissarov, S. S., \& Falle, S. A. E. G. 1997, MNRAS, 288, 833

Kouveliotou, C., Meegan, C. A., Fishman, G. J., et al. 1993, ApJL, 413, L101

Krolik, J. H., \& Piran, T. 2011, ApJ, 743, 134

Kulkarni, S. R., Frail, D. A., Wieringa, M. H., et al. 1998, Nature, 395, 663

Lazzati, D., \& Begelman, M. C. 2005, ApJ, 629, 903

Lazzati, D., Morsony, B. J., \& Begelman, M. C. 2009, ApJ, 700, L47

Lazzati, D., Morsony, B. J., \& Begelman, M. C. 2011, ApJ, 732, 34

Lazzati, D., Morsony, B. J., Blackwell, C. H., \& Begelman, M. C. 2012, ApJ, 750, 68

Lazzati, D., Morsony, B. J., Margutti, R., \& Begelman, M. C. 2013, ApJ, 765, 103 
Lazzati, D., Morsony, B. J., \& López-Cámara, D. 2015, Journal of High Energy Astrophysics, 7, 17

Levan, A. J., Tanvir, N. R., Starling, R. L. C., et al. 2014, ApJ, 781, 13

Levan, A. J. 2015, Journal of High Energy Astrophysics, 7, 44

Leventis, K., van Eerten, H. J., Meliani, Z., \& Wijers, R. A. M. J. 2012, MNRAS, 427,1329

Li, L.-X. 2007, MNRAS, 375, 240

Liang, E.-W., Zhang, B.-B., Stamatikos, M., et al. 2006, ApJ, 653, L81

Liang, E., Zhang, B., Virgili, F., \& Dai, Z. G. 2007, ApJ, 662, 1111

Livio, M., \& Waxman, E. 2000, ApJ, 538, 187

Longair, M. S. 2011, High Energy Astrophysics, Cambridge, UK: Cambridge University Press

Lyutikov, M., \& Blandford, R. 2004, Gamma-Ray Bursts in the Afterglow Era, 312, 449

MacFadyen, A. I., \& Woosley, S. E. 1999, ApJ, 524, 262

Mandal, S., \& Eichler, D. 2010, ApJ, 713, L55

Margutti, R., Soderberg, A. M., Wieringa, M. H., et al. 2013, ApJ, 778, 18

Margutti, R., Guidorzi, C., Lazzati, D., et al. 2015, ApJ, 805, 159

Marti, J. M. A., Muller, E., Font, J. A., \& Ibanez, J. M. 1995, ApJ, 448, L105 
Martí, J. M., Müller, E., Font, J. A., Ibáñez, J. M. Z., \& Marquina, A. 1997, ApJ, 479,151

Matheson, T., Filippenko, A. V., Chornock, R., Leonard, D. C., \& Li, W. 2000, AJ, 119,2303

Mathis, J. S., Rumpl, W., \& Nordsieck, K. H. 1977, ApJ, 217, 425

Matzner, C. D., \& McKee, C. F. 1999, ApJ, 510, 379

Matzner, C. D. 2003, MNRAS, 345, 575

Matzner, C. D., Levin, Y., \& Ro, S. 2013, ApJ, 779, 60

Mazzali, P. A., Deng, J., Nomoto, K., et al. 2006, Nature, 442, 1018

Mészáros, P., \& Rees, M. J. 1997, ApJ, 476, 232

Mészáros, P., \& Waxman, E. 2001, Physical Review Letters, 87, 171102

Metzger, B. D., Giannios, D., Thompson, T. A., Bucciantini, N., \& Quataert, E. 2011, MNRAS, 413, 2031

Metzger, B. D., Margalit, B., Kasen, D., \& Quataert, E. 2015, MNRAS, 454, 3311

Metzger, M. R., Djorgovski, S. G., Kulkarni, S. R., et al. 1997, Nature, 387, 878

Mignone, A., Plewa, T., \& Bodo, G. 2005, ApJS, 160, 199

Mizuta, A., Yamasaki, T., Nagataki, S., \& Mineshige, S. 2006, ApJ, 651, 960

Mizuta, A., \& Aloy, M. A. 2009, ApJ, 699, 1261

Mizuta, A., Nagataki, S., \& Aoi, J. 2011, ApJ, 732, 26 
Mizuta, A., \& Ioka, K. 2013, ApJ, 777, 162

Modjaz, M., Li, W., Butler, N., et al. 2009, ApJ, 702, 226

Morsony, B. J., Lazzati, D., \& Begelman, M. C. 2007, ApJ, 665, 569

Morsony, B. J., Lazzati, D., \& Begelman, M. C. 2010, ApJ, 723, 267

Nakar, E., \& Piran, T. 2002, MNRAS, 331, 40

Nakar, E. 2007, Phys. Rep., 442, 166

Nakar, E., \& Sari, R. 2010, ApJ, 725, 904

Nakar, E., \& Sari, R. 2012, ApJ, 747, 88

Nakar, E., \& Piro, A. L. 2014, ApJ, 788, 193

Nakar, E. 2015, ApJ, 807, 172

Nakauchi, D., Kashiyama, K., Suwa, Y., \& Nakamura, T. 2013, ApJ, 778, 67

Narayan, R., Paczynski, B., \& Piran, T. 1992, ApJ, 395, L83

Narayan, R., Piran, T., \& Kumar, P. 2001, ApJ, 557, 949

Narayan, R., \& Kumar, P. 2009, MNRAS, 394, L117

Nicholl, M., \& Smartt, S. J. 2016, MNRAS, 457, L79

Norris, J. P., Marani, G. F., \& Bonnell, J. T. 2000, ApJ, 534, 248

Paczynski, B. 1990, ApJ, 363, 218

Panaitescu, A., \& Kumar, P. 2002, ApJ, 571, 779 
Pastorello, A., Mattila, S., Zampieri, L., et al. 2008, MNRAS, 389, 113

Pe'er, A., \& Waxman, E. 2005, ApJ, 628, 857

Pe'er, A., Mészáros, P., \& Rees, M. J. 2006, ApJ, 652, 482

Pe'er, A. 2015, Advances in Astronomy, 2015, 907321

Pian, E., Mazzali, P. A., Masetti, N., et al. 2006, Nature, 442, 1011

Piran, T. 2004, Reviews of Modern Physics, 76, 1143

Piro, L. 2001, X-ray Astronomy: Stellar Endpoints, AGN, and the Diffuse X-ray Background, 599, 295

Piro, L., Troja, E., Gendre, B., et al. 2014, ApJL, 790, L15

Predehl, P., \& Schmitt, J. H. M. M. 1995, A\&A, 293, 889

Quataert, E., \& Shiode, J. 2012, MNRAS, 423, L92

Ramirez-Ruiz, E., Celotti, A., \& Rees, M. J. 2002, MNRAS, 337, 1349

Rees, M. J., \& Mészáros, P. 1992, MNRAS, 258, 41P

Rees, M. J., \& Meszaros, P. 1994, ApJ, 430, L93

Rees, M. J., \& Mészáros, P. 1998, ApJ, 496, L1

Rhoads, J. E. 1999, ApJ, 525, 737

Rybicki, G. B., \& Lightman, A. P. 1979, Radiative Processes in Astrophysics (New York: Wiley-Interscience)

Ryde, F. 2005, ApJ, 625, L95 
Sari, R., \& Piran, T. 1995, ApJ, 455, L143

Sari, R., Narayan, R., \& Piran, T. 1996, ApJ, 473, 204

Sari, R. 1997, ApJ, 489, L37

Sari, R., \& Piran, T. 1997, ApJ, 485, 270

Sari, R., Piran, T., \& Narayan, R. 1998, ApJ, 497, L17

Sari, R., Piran, T., \& Halpern, J. P. 1999, ApJ, 519, L17

Sari, R., \& Mészáros, P. 2000, ApJ, 535, L33

Senno, N., Murase, K., \& Mészáros, P. 2016, Phys. Rev. D, 93, 083003

Shao, L., Dai, Z. G., \& Mirabal, N. 2008, ApJ, 675, 507

Shen, R.-F., Willingale, R., Kumar, P., O’Brien, P. T., \& Evans, P. A. 2009, MNRAS, 393,598

Silverman, J. M., Nugent, P. E., Gal-Yam, A., et al. 2013, ApJS, 207, 3

Smith, N., \& Arnett, W. D. 2014, ApJ, 785, 82

Smolsky, M. V., \& Usov, V. V. 2000, ApJ, 531, 764

Soderberg, A. M., Kulkarni, S. R., Nakar, E., et al. 2006, Nature, 442, 1014

Sollerman, J., Jaunsen, A. O., Fynbo, J. P. U., et al. 2006, A\&A, 454, 503

Starling, R. L. C., Wiersema, K., Levan, A. J., et al. 2011, MNRAS, 411, 2792

Starling, R. L. C., Page, K. L., Pe'er, A., Beardmore, A. P., \& Osborne, J. P. 2012, MNRAS, 427, 2950 
Stratta, G., Gendre, B., Atteia, J. L., et al. 2013, ApJ, 779, 66

Taddia, F., Fremling, C., Sollerman, J., et al. 2016, arXiv:1605.02491

Tamborra, I., \& Ando, S. 2016, Phys. Rev. D, 93, 053010

Tan, J. C., Matzner, C. D., \& McKee, C. F. 2001, ApJ, 551, 946

Thompson, C. 2006, ApJ, 651, 333

Thöne, C. C., de Ugarte Postigo, A., Fryer, C. L., et al. 2011, Nature, 480, 72

Toma, K., Ioka, K., Sakamoto, T., \& Nakamura, T. 2007, ApJ, 659, 1420

Usov, V. V. 1992, Nature, 357, 472

Usov, V. V. 1994, MNRAS, 267, 1035

van Eerten, H., Zhang, W., \& MacFadyen, A. 2010, ApJ, 722, 235

van Eerten, H. J., \& MacFadyen, A. I. 2012, ApJ, 751, 155

van Eerten, H., \& MacFadyen, A. 2013, ApJ, 767, 141

van Paradijs, J., Groot, P. J., Galama, T., et al. 1997, Nature, 386, 686

Vink, J., Bleeker, J., van der Heyden, K., et al. 2006, ApJ, 648, L33

Wang, X.-Y., Li, Z., Waxman, E., \& Mészáros, P. 2007, ApJ, 664, 1026

Wang, P., Abel, T., \& Zhang, W. 2008, ApJS, 176, 467

Waxman, E., \& Draine, B. T. 2000, ApJ, 537, 796

Waxman, E., Mészáros, P., \& Campana, S. 2007, ApJ, 667, 351 
Woosley, S. E., \& Bloom, J. S. 2006, ARA\&A, 44, 507

Wygoda, N., Waxman, E., \& Frail, D. A. 2011, ApJ, 738, L23

Zhang, W., \& MacFadyen, A. 2009, ApJ, 698, 1261

Zhang, B., \& Mészáros, P. 2001, ApJL, 552, L35

Zhang, W., Woosley, S. E., \& MacFadyen, A. I. 2003, ApJ, 586, 356

Zhao, Y.-N., \& Shao, L. 2014, ApJ, 789, 74 